\title{
Simulations of Bosonic Dark Matter
}

\author{
Dissertation \\ for the award of the degree \\ "Doctor of Philosophy" \\ at Georg-August-Universität Göttingen \\ within the doctoral programme PROPHYS \\ of the Georg-August University School of Science (GAUSS)
}

submitted by

Jiajun Chen

from Zhejiang, China

Göttingen, 2021 


\section{Thesis advisory committee:}

Prof. Dr. Jens C. Niemeyer

Institut für Astrophysik, Georg-August-Universität Göttingen

Prof.Dr. David J. E. Marsh

Institut für Astrophysik, Georg-August-Universität Göttingen

Dr. Erik W. Lentz

Institut für Astrophysik, Georg-August-Universität Göttingen

\section{Members of the examination board:}

Referee: Prof. Dr. Jens C. Niemeyer

Institut für Astrophysik, Georg-August-Universität Göttingen

Co-referee: Dr. David J. E. Marsh

Institut für Astrophysik, Georg-August-Universität Göttingen

\section{Other members of the Examination Board:}

Prof. Dr. Wolfram Kollatschny

Institut für Astrophysik, Georg-August-Universität Göttingen

Prof. Dr. Stefan Dreizler

Institut für Astrophysik, Georg-August-Universität Göttingen

Prof. Dr. Ariane Frey

Physikalisches Institut, Georg-August-Universität Göttingen

Prof. Dr. Steffen Schumann

Institut für Theoretische Physik, Georg-August-Universität Göttingen

Day of the oral examination: 26. 04. 2021 


\section{Acknowledgements}

Firstly, I would like to thank Prof. Dr. Jens Niemeyer for giving me the chance to work on this interesting and challenging research project, for providing a good working atmosphere, and for his continuous support. His great academic freedom, constructive suggestions, and his desire to obtain the understanding of physics have been wonderful motivations. I would also like to thank Prof. Dr. David J. E. Marsh and Dr. Erik W. Lentz for co-supervising this dissertation. I would like to thank Xiaolong Du for his help in the past four years. I especially thank David Ellis for helping me revise the dissertation. I would like to thank everybody in our group, especially Katy Clough, Bodo Schwabe, Jan Veltmaat, Benedikt Eggemeier, Gaoyuan Wang, Jens Oltmanns, Felix Wiebe, Sebastian Hoof, Jurek Bauer, Christoph Behrens, Mona Dentler, Viraf Mehta and Salome Mtchedlidze for the great working atmosphere and a wonderful time in the office, and for being colleagues and friends.

J. Chen acknowledges the China Scholarship Council (CSC) for financial support. Finally, I want to thank my good friend Zhengwen. Liu, Zucheng. Chen, Dong. Yinyingying. Huo, Xianghua. Kong, Linfeng, $\mathrm{Li}$ and my family for their support. 


\begin{abstract}
Dark matter is a hypothetical form of matter, which is thought to make up nearly $27 \%$ of the contents in our Universe. An increasingly popular idea is that the dark matter could be composed of light (pseudo-)scalar particles with large occupation number so that they can be described by a classical scalar field $\phi$, with the mass $\approx 10^{-22} \mathrm{eV}$. As the finite energy ground state solutions for such a field, boson stars are a good subject in the study of dark matter. In this dissertation, the primary focus is on boson stars and their surrounding miniclusters. Firstly, using my new algorithms employing the Pseudo-Spectral method, I simulate the collision of two boson stars, and find the interference pattern when two boson stars overlap. The relationship between boson stars and the surrounding miniclusters are also introduced. Secondly, I study the formation and growth of boson stars in their surrounding miniclusters by gravitational condensation using the numerical method developed. Fully dynamical attractive and repulsive self-interactions are considered for the first time. In the case of pure gravity, I numerically prove that the growth of boson stars inside halos slows down and saturates as has been previously conjectured, and detail its conditions. Self-interactions are included using the Gross-Pitaevskii-Poisson equations. We find that in the case of strong attractive self-interactions the boson stars can become unstable and collapse, in agreement with previous stationary computations. At even stronger coupling, the condensate fragments. Repulsive self-interactions, as expected, promote boson star formation, and lead to solutions with larger radii. Lastly, I simulate the formation of vortices during the merger of boson stars with gravity and find that weak attractive self-interaction can be ignored in this process.
\end{abstract}




\section{Contents}

1 Introduction of Dark Matter 1

1.1 Discovery of dark matter $\ldots \ldots \ldots \ldots \ldots \ldots$

1.2 Observational evidence. . . . . . . . . . . . . . . . . . . . 4

1.2.1 Brief History of the Universe . . . . . . . . . . . . . . 4

1.2 .2 Cosmic microwave background $\ldots \ldots \ldots \ldots \ldots \ldots$

1.2 .3 Gravitational lensing . . . . . . . . . . . . . . . . 6

1.2 .4 Bullet Cluster . . . . . . . . . . . . . . . . . . . . . . 7

1.3 Candidate particles of dark matter $\ldots \ldots \ldots \ldots \ldots$

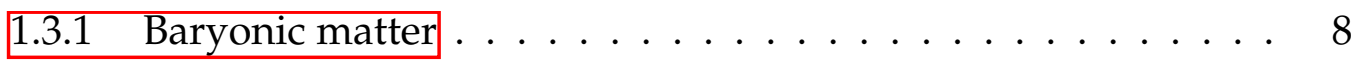

1.3 .2 Non-Baryonic matter . . . . . . . . . . . . . . . . . . . 9

$1.4 \quad \Lambda$-cold dark matter model $\ldots \ldots \ldots \ldots$. . . . . . . . . . . . . 9

1.4 .1 Cold dark matter halo . . . . . . . . . . . . . . . . . . 10

1.5 Candidates particles of cold dark matter . . . . . . . . . . . . 13

1.5 .1 Weakly interacting massive particles . . . . . . . . . . . . 13

1.5 .2 Ultralight bosons in cosmology . . . . . . . . . . . . . . . 14

1.5 .3 Axions . . . . . . . . . . . . . . . . . . . . 14

1.5 .3 .1 QCD axions . . . . . . . . . . . . . . 17

1.5.3.2 Axions in string theory $\ldots \ldots \ldots \ldots$

1.6 Detection of dark matter particles . . . . . . . . . . . . . . . . 18

1.6 .1 Detection of Axion dark matter . . . . . . . . . . . . . . . . 19

1.6 .2 Thesis Overview . . . . . . . . . . . . . . . . . . 22

2 Boson stars and numerical methods 23

2.1 Boson stars . . . . . . . . . . . . . . . . . . . . . . . 23

2.1 .1 Compact boson stars . . . . . . . . . . . . . . . . 23

2.1 .2 Non-compact boson stars . . . . . . . . . . . . . . . . . . 24

2.2 Soliton solutions to the GPP equations . . . . . . . . . . . . 26

2.3 Dynamical numerical simulations of ultralight bosons . . . . . . . 33 
2.4 Relationship of halos and non-compact boson stars . . . . . . . . . 33

2.5 Pseudo-Spectral Method . . . . . . . . . . . . . . . . . 37

2.5 .1 Second Order Algorithm . . . . . . . . . . . . . . . 38

2.5 .2 Fourth Order Algorithm . . . . . . . . . . . . . . . . . 39

2.5 .3 Fast Fourier transform . . . . . . . . . . . . . . . . . . 39

2.6 Application of codes . . . . . . . . . . . . . . . . . . . 40

2.6 .1 Quantum harmonic oscillator . . . . . . . . . . . . . . 40

2.6 .2 Colliding of two boson stars . . . . . . . . . . . . . . 41

2.6 .3 Code accuracy . . . . . . . . . . . . . . 42

2.7 Conclusion . . . . . . . . . . . . . . . . . . 44

3 Formation and evolution of boson stars and halos/miniclusters 47

3.1 Theory of condensation of bosons . . . . . . . . . . . . . . . 47

3.1 .1 Transport Rutherford cross section . . . . . . . . . . . . . . . . 49

3.2 Simulation of condensation of Bosons . . . . . . . . . . . . . 50

3.2 .1 Initial conditions . . . . . . . . . . . . . . . 50

3.2.2 Formation of miniclusters and condensation of bosons stars with only gravity . . . . . . . . . . . . . . . 50

3.2.3 Saturation of boson stars in axion miniclusters . . . . . . . . . 52

3.3 Axions with self interaction . . . . . . . . . . . . . . . . . 59

3.3.1 Axions with weak attractive self interaction. . . . . . . . . . . 59

3.3.2 Bosons with repulsive self-interactions . . . . . . . . . . 61

3.4 Formation of multiple boson stars . . . . . . . . . . . . . . . . 64

3.5 Conclusions . . . . . . . . . . . . . . . . . . . . . 69

$4 \quad$ Turbluence creation in the process of merger of boson stars 71

4.1 Fluid Formulation . . . . . . . . . . . . . . . . . 73

4.1 .1 Power spectrum of turbulence . . . . . . . . . . . . . 74

4.2 Turbulence in merges of boson stars . . . . . . . . . . . . . 75

4.2 .1 Merger of boson stars . . . . . . . . . . . 76

4.2 .2 Turbulence in halos $/$ miniclusters . . . . . . . . . . . . . . . 76

4.2.3 Comparison between second-order and fourth-order algo-

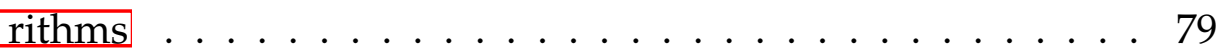

4.3 Conclusion . . . . . . . . . . . . . . . . . . . . 82

$\begin{array}{llr}5 & \text { Outlook } & 84\end{array}$ 
Bibliography 


\section{List of Figures}

1.1 The points are the rotation curve of spiral galaxy Messier 33. The

\begin{tabular}{|c|c|}
\hline & ous line is the fitting model. dashed-dotted line, short dashed \\
\hline & id long dashed line are the halo contribution, the stellar disk \\
\hline & and the gas contribution respectively. Reproduced from [47]. . . . . . 3 \\
\hline 1.2 & Cosmic background radiation observed by WMAP in 2012. Repro- \\
\hline & duced from $[175[. \ldots \ldots \ldots \ldots \ldots$ \\
\hline 1.3 & X-ray photo of the Bullet Cluster taken by Chandra X-ray Observa- \\
\hline & tory. Reproduced from $[109]]$. \\
\hline 1.4 & Dark matter halo from cosmological N-body simulations. Repro- \\
\hline & duced from $[174[. . .$. \\
\hline 1.5 & The size of core in a warm dark matter halo of mass $M=5 * 10^{8} M_{\odot}$ \\
\hline & as a function of warm dark matter thermal relic mass. Reproduced \\
\hline & from Ref. [104,[105[. . . \\
\hline 1.6 & Summary of probes and constraints of axion cosmology. Repro- \\
\hline & duced from Ref. [112].] \\
\hline 1.7 & A small sample of some of the existing (solid) and proposed (trans- \\
\hline & parent) experimental constraints on the axion-photon coupling. The \\
\hline & yellow band indicates the range of parameter space in which differ- \\
\hline & ent models of the QCD axion predict the particle to be. Produced \\
\hline & using the public code provided by Ref. [132] \\
\hline
\end{tabular}

2.1 Total mass of boson star with attractive self-interactions $(g<0)$ as a

function of the central density. . . . . . . . . . . . . . 28

2.2 Core radius of boson star with attractive self-interactions $(g<0)$ as

\begin{tabular}{|c|}
\hline a function of the central density. Circles: numeric results. Solid line: \\
\hline
\end{tabular}
fitting function, Eq. 2.27 . . . . . . . . . . . . . . . . . . . 29

2.3 Parameter $\alpha$ in the fitting formula Eq. 2.26 for the case with attractive self-interactions $(g<0)$. Circles: numeric results. Solid line: fitting function, Eq. 2.28 . . . . . . . . . . . . . . . . 30 
2.4 Parameter $\beta$ in the fitting formula Eq. 2.26 for the case with attractive interactions $(g<0)$ as a function of the central density. Solid line: fitting function, Eq. 2.29. . . . . . . . . . . . . . . . . 31

2.5 Core radius of boson star with repulsive self-interactions $(g>0)$ as a function of the central density. Circles: numeric results. Solid line: fitting function, Eq. 2.30| . . . . . . . . . . . . . . . . . . 31

2.6 Parameter $\alpha$ in the fitting formula Eq. 2.26 for the case with repulsive self-interactions $(g>0)$ as a function of the central density. Circles: numeric results. Solid line: fitting function, Eq. 2.28 . . . . . . . . . . 32

2.7 Snapshots of boson stars collision simulation. Panel (a) shows the \begin{tabular}{|l|}
\hline density projection at the initial stages. Panels (b), (c) show the den- \\
\hline sity projection at the intermediate stages. Panel (d) shows a dense \\
\hline
\end{tabular} core stay at the center of the halo. Reproduced from [155]. . . . . . . 35

2.8 The left panel shows the density profile of halos. Dashed lines are \begin{tabular}{|c|}
\hline the examples at different redshifts at $0<z<12$. The solid lines \\
\hline
\end{tabular} are soliton solution, see Eq 2.25 . Dot-dashed line is the NFW profile given by Eq. 1.2 the right panel shows core-halo mass relation. Reproduced from Ref. [155] . . . . . . . . . . . . . . . . . . . . 36

2.9 Solid line is the radial density profile of halo with ultra-light bosons. Dashed line is the profile of halo with no ultra-light bosons, i.e. not quantum effect. The left panel is a dwarf-size halo, $M_{\text {halo }}=10^{10} M_{\odot}$, and the right panel is a Milky-Way size halo, $M_{\text {halo }}=10^{12} M_{\odot}$. Reproduced from Ref. [147] . . . . . . . . . . . . . . . . . . . 36

2.10 Range of $\alpha$. Reproduced from Ref. [147]. . . . . . . . . . . . . . . . . . 37

2.11 Comparison of the initial condition $|\widetilde{\psi}(\widetilde{x}, \widetilde{t} \sim 0)|$ (black dotted line) and simulation results $|\widetilde{\psi}(\widetilde{x}, \tilde{t} \sim 100)|$ (red solid line) $\ldots$. . . . . . . . 41

2.12 Collision of two boson stars. This shows the density evolution $|\widetilde{\psi}(\widetilde{x}, 0,0, \widetilde{t})|^{2}$.

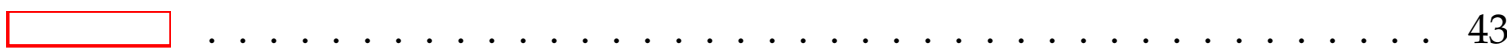

2.13 Comparison of the theoretical and simulation results of $|\widetilde{\psi}(\widetilde{x}, 0,0, \widetilde{t})|^{2}$ at $t \sim 5$. The red line is the theoretical results and blue line is simulation results. . . . . . . . . . . . . . . . . . . . . 44

2.14 Relative errors of the total energy with respect to time for different order algorithm. . . . . . . . . . . . . 45 
3.1 Formation of Bose star from random field with initial distribution from $\psi(\vec{x}, 0) \propto e^{-\widetilde{p}^{2}}$. Total mass $\widetilde{N}=628.3$, box size $\widetilde{L}=125$. Left: Projection of density at $\widetilde{t}=0$. Right: Projection of density at $\widetilde{t}=$

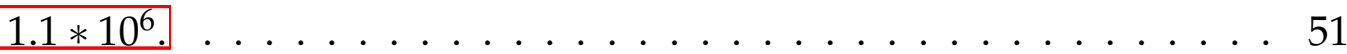

3.2 Formation and growth of Bose star from random field with initial distribution from $\psi(\vec{x}, 0) \propto e^{-\tilde{p}^{2}}$. Total mass $\widetilde{N}=628.3$, box size $\widetilde{L}=125$. Left: Radial profile of the object (colored dots) in Fig. 3.1 solitonic profiles (solid lines) as given by Eq. 2.25 with the same central densities at $\widetilde{t}=1.1 * 10^{6}$. Right: Maximum of $\widetilde{\psi}$ over the box as a function of time. . . . . . . . . . . . . . . . . . 52

3.3 Snapshots of the density field from one simulation with $\tilde{N}=1005.3$, $\widetilde{L}=18$. (a) Projected density at the initial time. (b) Projected density at $\widetilde{t}=10$, which shows that minicluster is forming in the box. (c ) Projected density at $\widetilde{t}=30$. (d) Projected density at $\widetilde{t}=200$. A single dense object is visible at the centre of the minicluster. . . . . . 53

3.4 Density profiles of the minicluster at different times (colored dots) compared with solitonic profiles (solid lines) as given by Eq. 2.25 with the same central densities. . . . . . . . . . . . . . . . . . . 54

3.5 Time to Bose star formation in the cases of Gaussian and Delta initial

\begin{tabular}{|c|c|}
\hline & istributions. Note that the $\tau_{g r}$ of Gaussian initial distributions are \\
\hline & shifted upwards $\left(\tau_{g r} \rightarrow \tau_{g r} * 10\right)$ for clearly purposes. The dotted \\
\hline & line is real $\tau_{g r}$ obtained by Eq. 3.11 \\
\hline 3.6 & The mean stacked mass of boson stars evolution (solid lines) for dif- \\
\hline & ferent box sizes $\widetilde{L}=25,20,18,15$ and total mass $\widetilde{N}=691,754,817,880,942$ \\
\hline & The data from simulation with the same box size $\widetilde{L}$ but different to- \\
\hline & tal mass $\widetilde{N}$ are divided into 500 time bins. The shaded regions show \\
\hline & the $1-\sigma$ intervals. The time and mass of boson stars are normalized \\
\hline & by the condensation time, $\tau_{\text {gravity }}$ and the total mass, $\widetilde{N}_{691}^{1 / 3}$, where \\
\hline & $\tilde{N}_{691}=\tilde{N} / 691$. Note that here $\tau_{\text {gravity }}$ is computed using Eq. (3.11) \\
\hline & for the initial configuration, i.e. $R=L, v=v_{0}$, and $n=N / L^{3}$, to \\
\hline & avoid ambiguities in the definitions of halo radius and density. . . \\
\hline 3.7 & Mean fractional deviation from the fitting formula $\widetilde{M}_{* \text {,sat }} \propto \widetilde{N}^{\alpha}$. The \\
\hline & the results from simulations grouped by box size, \\
\hline
\end{tabular}


3.8 Snapshots of the density field from one simulation with $\widetilde{N}=1005.3$, $\widetilde{L}=18, \widetilde{g}=-0.007$. (a) Projected density at the initial time. (b) Projected density at $\widetilde{t}=10$, which shows that minicluster is forming in the box. (c) Projected density at $\widetilde{t}=30$. (d) Projected density at $\widetilde{t}=48$. Compared with the case without self-interactions, the boson star formed at the center is denser. . . . . . . . . . . . . . . 60

3.9 Density profiles of the minicluster at different times (colored dots) from simulations of the GPP equations with $\widetilde{g}=-0.007$. Solitonic

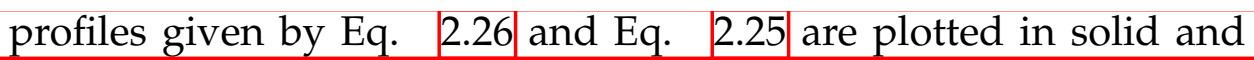
dashed lines, respectively. . . . . . . . . . . . . . . . 61

3.10 Maximum density growth with respect to time from simulations assuming different self-interaction couplings: $\widetilde{g}=0$ (without selfinteractions), $\widetilde{g}=-0.000005, \widetilde{g}=-0.001, \widetilde{g}=-0.007, \widetilde{g}=-0.01$, and $\widetilde{g}=-0.012$. The box size $\widetilde{L}=18$ and the total mass $\widetilde{N}=1005.3$. The horizontal dashed lines mark the densities which satisfy $\widetilde{\rho} \widetilde{g}^{2}=$ 0.52 , when the boson star will collapse. . . . . . . . . . . . . . 62

3.11 Snapshots of the density field at $\widetilde{t}=200$ from simulations in a box of size $\widetilde{L}=18$ and total mass $\widetilde{N}=1005.3$ assuming different $\widetilde{g}$. . . . . 63

3.12 Density profiles of the miniclusters from simulations (colored dots), compared with solitonic profiles (solid lines) as given by Eq. 2.25 and Thomas-Fermi approximation [108] [Eq. (2.31)] with the same central densities. . . . . . . . . . . . . . . . . . . . . . . 64

3.13 Left: Mass growth of boson stars with respect to time from simu\begin{tabular}{|l|}
\hline lations assuming different $\widetilde{g}$ (Note that the case with $g=10$ is not \\
\hline shown since no boson star was formed). Right: Maximum density \\
\hline growth with respect to time from simulations assuming different $\widetilde{g}$. \\
\hline The box size and total mass were $\widetilde{L}=18$ and $\widetilde{N}=1005.3$ respectively.
\end{tabular} 65

3.14 Snapshots of the density field from simulations of the GPP equations in a box of size $\widetilde{L}=18$, and total mass $\widetilde{N}=1005.3$. The selfinteraction coupling constant $\widetilde{g}=-0.04$. Note that due to resolution limit, we can not resolve the central region of the densest object, so we cutoff the projected density in the plot at $\approx 1500 .$. . . . . . . . . 67

3.15 Snapshots of the density field from simulations of the GPP equations (left column) and GP equations (right column). We pick $\widetilde{g}=-1$ (first row) and $\widetilde{g}=-80$ (second row). The box size $\widetilde{L}=18$, and the total mass $\widetilde{N}=1005.3$. . . . . . . . . . . . . . . . . . 68 
4.1 The transition from the laminar flow to turbulence flow. Reproduced from $[31] . \ldots \ldots \ldots \ldots$. . . . . . . . . . . . . . . . . . .

4.2 The left panel is snapshots of the density field from one simulation with 10 same boson stars in different positions. The box size $\widetilde{L}=40$ and the total mass $\widetilde{N}=258$. (a) Projected density at the initial time. (b) (c) Projected density at $\widetilde{t}=10$ and $\widetilde{t}=30$, which shows several boson star has merged together. (d) Projected density at $\widetilde{t}=200$. All boson star meger together. A single dense object is visible at the centre of the dark matter halo. The right panel is density profiles of the minicluster at $\widetilde{t}=200$ (colored dots) compared with solitonic profiles (blue lines) as given by Eq. 2.25 with the same central den-

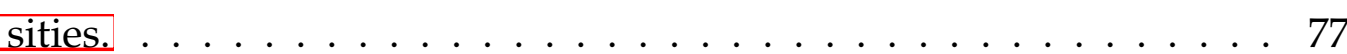

4.3 The left panel shows Snapshots of the density field from one simulation with 10 same boson stars. The box size $\widetilde{L}=40$ and the total mass $\widetilde{N}=258$. (a) Projected density at the initial time. (b) (c) Projected density at $\widetilde{t}=10$ and $\widetilde{t}=30$, which shows several boson star has merged together. (d) Projected density at $\widetilde{t}=200$. The right panel shows the density profile of the minicluster at $\widetilde{t}=200$ (colored dots) compared with solitonic profile, $\widetilde{g}=-0.007$, (blue lines) with the same central densities. . . . . . . . . . . . . . . . . . 77

4.4 The left panel is the density slice of the minicluster at $\widetilde{t}=200$. The middle panel shows the wavefunction phase $S$ at $\widetilde{t}=200$. The right panel is 3D volume rendering of the vortex line at $\widetilde{t}=200$. The box size $\widetilde{L}=40$ and the total mass $\widetilde{N}=258$. . . . . . . . . . . . . . . 78

4.5 Zooming-in projection of $1 /|\psi|^{2}$ for bosons with gravity. The annihilation of vortex line are shown. . . . . . . . . . . . . . . 78

4.6 The left panel is the velocity power spectra at different times after the merger of 10 same boson stars for boson with only gravity. Here we set the box size $\widetilde{L}=40$ and the total mass $\widetilde{N}=258$. The right panel shows the velocity power spectra from different initial conditions. Here we choose several results with different number of boson stars and total mass, $\widetilde{N}$. . . . . . . . . . . . . . . . . . . . 79

$4.7 \quad$ The left panel is the density slice of the minicluster at $\widetilde{t}=200$. The middle panel is the wavefunction phase $S$ at $\widetilde{t}=200$. The right panel is 3D volume rendering of the vortex line at $\widetilde{t}=200$. Here we set $\widetilde{g}=-0.007$, the box size $\widetilde{L}=40$ and the total mass $\widetilde{N}=258$. . . . 79 
4.8 Zooming-in projection of $1 /|\psi|^{2}$ for bosons with gravity and weak attractive self-interaction. We can see the annihilation of vortex line. Here, we set $\widetilde{g}=-0.007 . \ldots \ldots \ldots$. . . . . . . . . . . 80

4.9 The left panel is the velocity power spectra of merger of 10 same boson stars at different time. Here we set $\widetilde{g}=-0.007$, the box size $\widetilde{L}=40$ and the total mass $\widetilde{N}=258$. The right panel is velocity power spectra of merger of 10 same boson stars for different $\widetilde{g}$. Here we set the box size $\widetilde{L}=40$, all of boson stars with same center den-

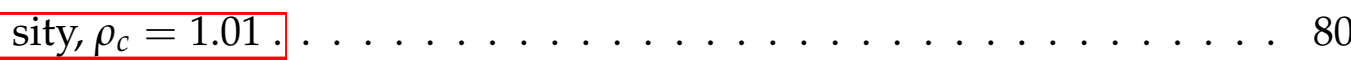

4.10 Relative errors of the total energy with respect to time for different algorithms. The left panel is the evolution of bosons with no selfinteraction, the right panel is the evolution of bosons with weak selfinteraction, $\widetilde{g}=-0.007 . \ldots \ldots \ldots \ldots \ldots 1$ 


\section{Chapter 1}

\section{Introduction of Dark Matter}

\subsection{Discovery of dark matter}

Dark matter is a hypothetical form of matter in the Universe. Through a large number of observations, modern astronomers have found many astronomical evidences for the existence of dark matter in our Universe. Through high-precision measurements, astronomers have concluded that ordinary matter and energy only accounts for around $5 \%$ of total mass-energy of our Universe, while the proportion of dark matter and dark energy are $27 \%$ and $68 \%$ respectively [142]. The existence of dark matter can solve the problems of inconsistency in the Big Bang theory [7]. The structure and evolution of galaxies are also affected by dark matter. Studies have shown that dark matter is perhaps to be composed of one or several new particles beyond the standard model of particle physics.

However, dark matter nearly does not interact with electromagnetic fields, which means it does not reflect, absorb and emit light. This is why it is called 'dark matter'. Therefore, dark matter is very difficult to detect directly. In fact, although the direct detection of dark matter has been going on for many years [5, 13, 194], such as the China Jinping Underground Laboratory, so far no research institute has been successful detect it directly. All of our understanding of dark matter come from indirect observations. Therefore, uncovering the nature of dark matter is one of the greatest challenges in modern physics and cosmology.

The study of dark matter has an elaborate history. In 1933, astrophysicist Fritz Zwicky used spectral redshift to measure the velocity of each galaxy relative to the galaxy cluster in the Coma system. Using the virial theorem, he discovered that the velocity dispersion of these galaxies were too high, and the gravity from visible mass in the galaxy cluster cannot confine these galaxies in the galaxy cluster. He attributed this phenomenon to the existence of unseen, dark matter [200]. 
Similar results were discovered by Sinclair Smith, who studied the mass of the Virgo cluster and Erik Holmberg, who analysed systems of galaxies [79, 167]. In the late 1950s, after new astronomical surveys [4, 159, 201], debates on these observations began. Some arguments provide new explanation for these awkward observations. For example, Ambartsumian debated that the lack of 'dynamical equilibrium' in these clusters was the reason of observed discrepancies. The galaxies with unusually fast speed were actually rapidly flying apart [10]. However, this theory had a huge problem. If groups and clusters of galaxies were unstable, their existence time could not exceed $10^{7}$ years [181]. In fact, in late 1960s and early 1970s, various theories were proposed to explain the phenomenon of cluster discrepancies [49, 64, 84, 184, 192]. It was very difficult to force a consensus since the observational and theoretical constraints were too few. Thus the existence of dark matter was just one possibility among alternatives at that time.

However, these disputes gradually disappeared after flat rotation curves were found by Rubin and Ford in 1970. The rotation curve of a disc galaxy is a tool to study the kinematics of galaxies, which is the orbital velocity of visible gas and stars in that galaxy versus their radial distance from the center of galaxy. It provides a way to estimate masses of galaxies [18, 62, 102, 118, 133]. Usually, according to Kepler's laws, the rotation velocity gas or stars, $v=\sqrt{G M(r) / r}$. Therefore, at radii greater than the bulk of the galaxies mass, such as that of our solar system, we expect the velocity of gas and stars to decrease with increasing distance from the center of the galaxy, see Fig. 1.1. Thus the rotation curve at the position of our solar system is in decline. However, in 1970, Rubin and Ford discovered that galaxy rotation curves tended to flatten at these large radii. This means that there is more gravity generated by invisible matter to make these gas and stars bounded. After that, flat rotation curves were verified by many observations of galaxies [48, 51, 89, 148, 161]. This provided strong additional evidence for the existence of dark matter.

Since the 1980s, a series of of observations were made which supported the presence of dark matter as well. These included gravitational lensing of background objects by galaxy clusters [127, 173, 193], the pattern of anisotropies in the cosmic microwave background (CMB) [78, 143, 165], and Sky surveys and baryon acoustic oscillations [97, 139].

Although this invisible particle remains unobserved, its existence became the consensus of the astronomical community since the 1980s. 


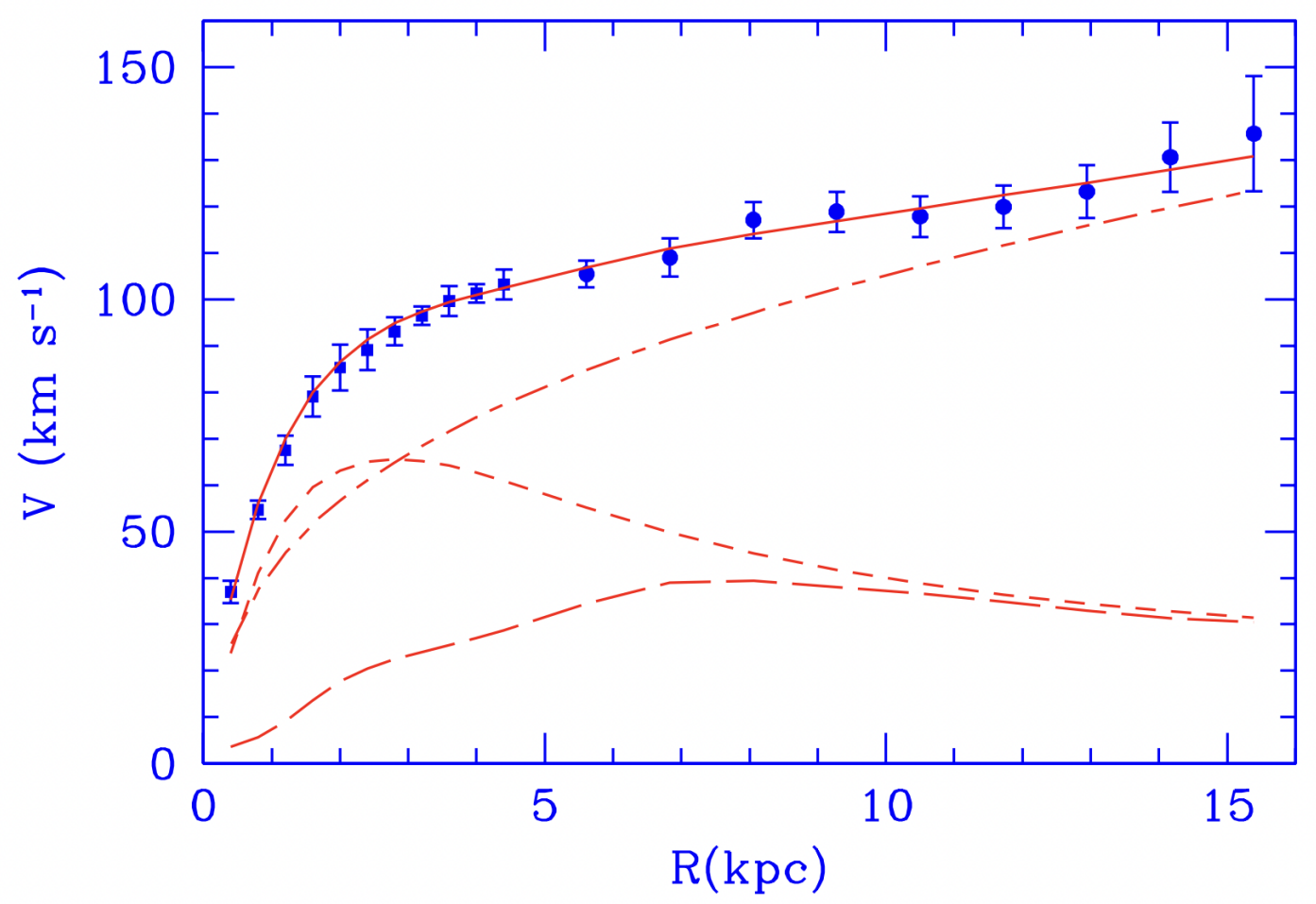

Figure 1.1: The points are the rotation curve of spiral galaxy Messier 33. The continuous line is the fitting model. dashed- dotted line, short dashed line and long dashed line are the halo contribution, the stellar disk and the gas contribution respectively. Reproduced from [47]. 


\subsection{Observational evidence}

Flat rotation curves are not the only evidence of existence of dark matter. Over the past decade, various additional observations have also support its existence. Dark matter roughly compose a quarter of the total mass and energy in the Universe. These observations include weak gravitational lensing measurements and measurements of the anisotropies of the CMB radiation as well as others.

\subsubsection{Brief History of the Universe}

One evidence of existence of dark matter comes from measuring the expansion history of the Universe [80]. This has been done through many observations [2, 34 66, 83, 129, 136, 145, 149, 150, 179, 190]. We know the matter components can slow down the expansion of the Universe by gravity. Thus the average density can be obtained by measuring the expansion of the Universe. Compare this average density with visible matter we already observed, we can know whether dark matter exists.

We are able to uncover much about the expansion history of the Universe through the observation of type Ia supernovae. Type Ia supernovae is a special type of supernovae, which appears in binary star systems. One of the two stars is a white dwarf. The other one can be anything such as a giant star or a white dwarf.

The type Ia category of supernova produces a fairly consistent peak luminosity since a white dwarf will explode at a critical mass. Their consistent peak luminosity allows these explosions to be regarded as standard candles. Since, the decrease in brightness is proportional to the square of distance, we can estimate the distance to the object from its brightness given its intrinsic luminosity. On the other hand, from supernova of spectrum, we can obtain its redshift. The redshift illustrates how much the Universe has expanded since the supernova explosion. The information of expansion history can be obtained by comparing the expansion factor with the distance to different supernovae. It is possible to infer from this the average matter density.

In 1998, the High-Z Supernova Search Team [146] and the Supernova Cosmology Project [140] made similar discoveries independently. They found that the expansion of the Universe is accelerating, and that the Universe contains approximately $75 \%$ of dark energy and $25 \%$ of matter. Since the composition of baryonic matter estimated from cosmic nucleosynthesis is $<5 \%$, this results suggests the that most of the matter in the Universe is 'dark'. 
Searching for the signature from Baryon Acoustic Oscillations (BAOs) is another method to measure the expansion history. In the early universe, photons and matter existed in a primordial plasma before recombination [137, 171]. The photons traveling were limited. But the Universe became neutral after recombination. The Photons diffused away since they were not interacting with the matter. Therefore, the sound wave was frozen and leave behind an overdense shells of matter with a fixed radius i.e. we call it sound horizon [61]. This overdense area eventually formed many galaxies. At this scale, the density field of galaxy have the peak number. And because of the expansion of the Universe, the peak would be moved to a larger scale. Comparing this scale with the sound horizon at recombination, we can obtain information of the expansion history. Similarly, the measurement of BAOs support the existence of dark matter [6, 196].

\subsubsection{Cosmic microwave background}

Shortly after the big bang, our Universe contained a dense white-hot cloud of hydrogen plasma. Plasma and radiation filled the entire Universe and, as the Universe expanded, they gradually cooled down. After the Universe dropped to a certain temperature, neutral atoms could be formed by the combination of protons and electrons. Thermal radiation could not be absorbed by these neutral atoms. That is, photons begin to travel through the entire space freely, rather than collide in the plasma composed of electrons and protons. However, due to the expansion of space, the wavelength increases over time leading to a decrease in temperature. This is the CMB.

Measurements of the $\mathrm{CMB}$, such as Wilkinson Microwave Anisotropy Probe (WMAP) [168], show that it is almost isotropic. This means that there are only very small variations in it's temperature across the sky. However, these small variations contain much important information about the Universe. We know that ordinary matter in early Universe was ionized and interacted with radiation by Thomson scattering. However, during this period, dark matter only interacted with radiation very weakly if at all. This means that the CMB was affected by dark matter differently. The density and and velocity of ordinary matter will be affected as well. Therefore, the dark matter and ordinary matter will leave different imprints in CMB. Since Arno Penzias and Robert Woodrow Wilson first discovered the CMB in 1964 [138], further measurements of the CMB have provided additional evidence for the existence of dark matter. For example, Balloon Observations Of Millimetric Extragalactic Radiation and Geophysics (BOOMERanG 


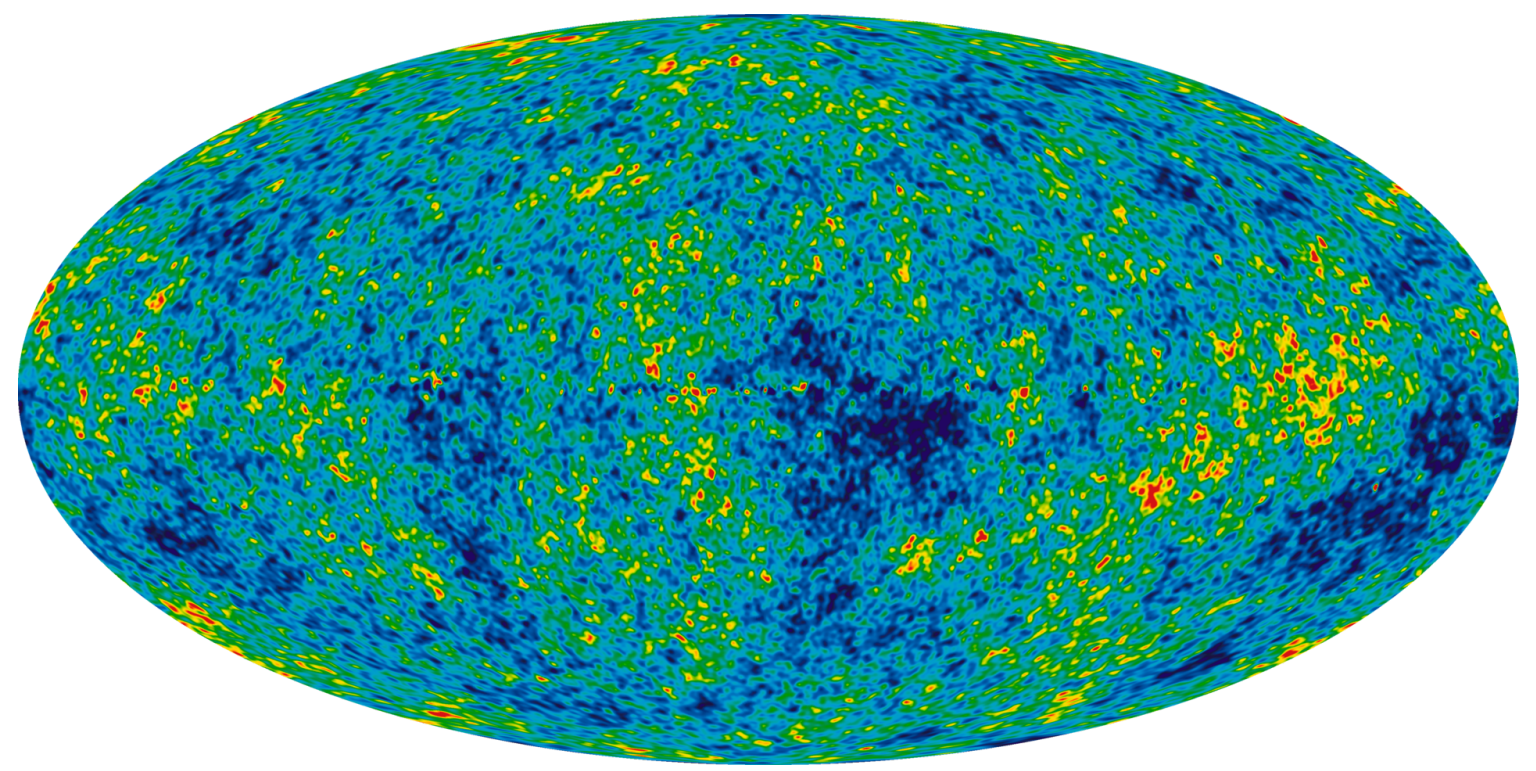

Figure 1.2: Cosmic background radiation observed by WMAP in 2012. Reproduced from [175].

experiment) [52], in 2000, discovered the first acoustic peak. After that, the power spectrum was observed by Wilkinson Microwave Anisotropy Probe (WMAP) in 2003-2012 precisely [168]. More precise results were obtained by the Planck spacecraft in 2013-2015. Theses observations found that dark matter constitutes about $26 \%$ of the energy density of the Universe while baryonic matter makes up only around $5 \%$.

\subsubsection{Gravitational lensing}

According to general relativity, when light emitted by a background light source passes through a gravitational field from massive object such as galaxy, galaxy cluster, and black hole, the light will bend like in a lens. We call this phenomenon gravitational lensing. The strength of the gravitational field determines the degree of light bending. Analyzing the distortion of the background light source enables us to study the nature of the gravitational field along line of sight.

The strong lensing analysis measures the curvature of rings of light formed from the distorted image of background sources, known as Einstein rings, and the position of multiple images to estimate the mass of the lens. The weak lensing analysis can be used to estimate the mass distribution of celestial bodies on a large scale through the statistical analysis of a large number of background source 
images, and is considered to be the best method of measuring dark matter in cosmology.

In fact, the Sloan Digital Sky Survey (SDSS) has stacked the signals of around a third of a million galaxies to reveal that baryons compose only 10 percent of the mass of most galaxies [28]. The Hubble Space Telescope SLACS survey also measures the mass distribution of throughout galaxies using gravitational lensing. All results indicate that dark matter composes about $26 \%$ of matter in our Universe.

\subsubsection{Bullet Cluster}

Modified gravity, such as Modified Newtonian dynamics (MOND), $F(R)$ or $F(T)$ gravity theory, as the name suggests, attempts to explain phenomena such as the galaxy rotation curves by changing the way gravity works [29, 33, 37, 85, 169, 197. [199]. However, the observation of Bullet Cluster challenge this theory.

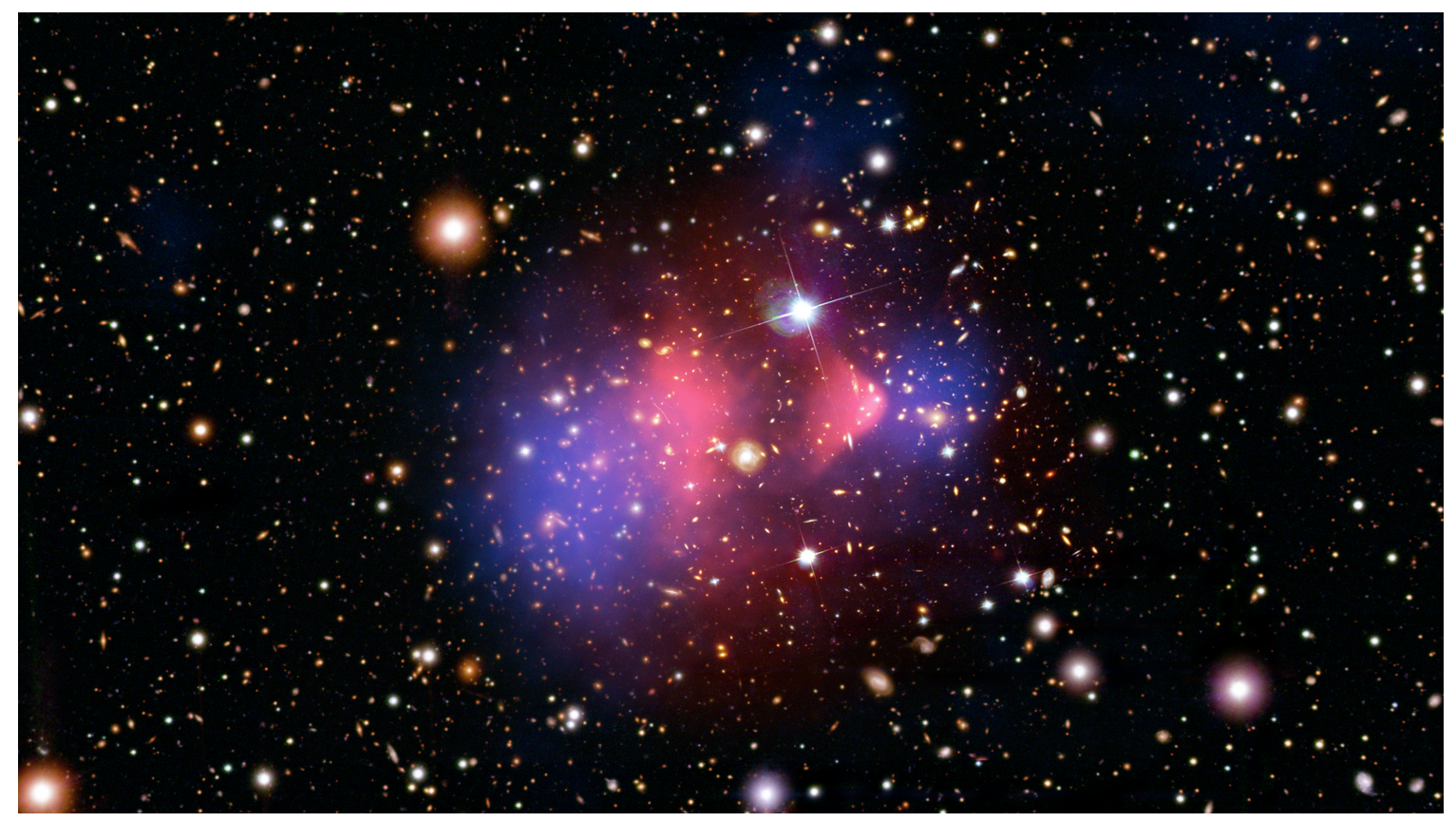

Figure 1.3: X-ray photo of the Bullet Cluster taken by Chandra X-ray Observatory. Reproduced from [109].

The Bullet Cluster is composed of two colliding galaxies clusters. The behaviour of gas, the putative dark matter and cluster pair stars are different during collision, which provide us the chance to study them separately. During the process of collision, the stars of galaxies are not effected greatly. But the gases could interact electromagnetically, which make the gases in two clusters much more slower 
than the stars. And in this process, the mass distribution required to explain the lensing effects can be estimated. By comparing the mass distribution required by lensing effects with the distribution of visible matter, we can check whether a theory, either the dark matter model or the modified gravity, can explain observations self-consistently. This observation is against the theories of modified gravity, because in these theories, the lensing would be follow the baryonic matter. But the observation showed that the mass distribution required by lensing is bimodal with peaks much closer to the centers of the two separate galaxy clusters instead a single peak at the center of baryonic matter. On the other hand, according the dark matter theory, we know dark matter only has gravity interaction. Thus, if we assume most of the gravitation clusters come from two regions of dark matter. And during the collision, they bypass the gas regions. This observation phenomenon of Bullet Cluster can be well explained [46, 106, 111].

\subsection{Candidate particles of dark matter}

Despite the fact that that so many evidences support the existence of dark matter, its composition remains unknown. Now, various particles are being investigated as possible candidates of dark matter, including sterile neutrino [57], little Higgs and so on. Based on this, many dark matter models have been developed.

\subsubsection{Baryonic matter}

Some researchers claim baryons are the dark matter. The main reason is that baryonic matter not only make up observable stars, such as planets and supernova, but also includes less common objects, like black holes, brown dwarfs, neutron stars and so on. These objects are very difficult to detect. However, several pieces of evidence indicate that the majority of dark matter is not made of baryons:

- Through gravitational microlensing observations in the Milky Way, astronomers have determined that only a small fraction of the dark matter can be in this form [72, 126, 126, 176, 195].

- When backlit by stars, baryonic gas or dust is visible. Thus the trace of these invisible objects still can be found as well. But the total mass of these objects is much less than the estimation of mass of dark matter in galaxies clusters. 
- According to the predictions of the big bang theory, more baryons means more heavy elements being synthesized during the Big Bang. To agree with the observed abundances we require that baryonic matter is about $4 \%$ of the critical density of the Universe. But the observations indicate that the total invisible matter density make up about $30 \%$ of the critical density.

\subsubsection{Non-Baryonic matter}

Another possibility is that dark matter is composed of non-baryonic matter. Maybe you can say "non-baryonic matter isn't required to interact as strongly, if at all via electromagnetism." Thus it can exist as various forms rather than invisible dense objects. Hypothetical particles, such as gravitationally-interacting massive particles (GIMPs), sterile neutrinos, weakly interacting massive particles (WIMPs), supersymmetric particles, axions, are some of the most popular candidate particles of non-baryonic dark matter [53, 57, 134].

On the other hand, non-baryonic matter revealed by its gravitational effects since they nearly do not interact with the electromagnetic field. However, some candidate particles can annihilate with themselves. This process perhaps produce observable signal such as gamma rays. Of course, these provide good ways to detect them [26].

\section{$1.4 \quad \Lambda$-cold dark matter model}

$\Lambda$-cold dark matter model is a parametrization of the Big Bang cosmological model. $\Lambda$-cold dark matter model is the simplest model so far. It can explain many properties of the Universe:

- the existence and structure of CMB;

- the large-scale structure of the distribution of galaxies;

- the The relative abundances of hydrogen, helium, and lithium in the Universe;

- the accelerating expansion of the Universe observed by distant galaxies and supernovae. 
In $\Lambda$-cold dark matter model, $\Lambda$ is the cosmological constant. It is associated with a vacuum energy or dark energy. Dark energy can explain the current accelerating expansion of the Universe. According to the general relativity, when expansion of the Universe is accelerating, the cosmological constant acts as a negative pressure, $p=-\rho c$, where $c$ is speed of light and $\rho$ is dark energy density. $\Lambda$ cold dark matter model is based on at least 6 parameters [143]:

\begin{tabular}{ccc}
\hline & $g<0$ & $g>0$ \\
\hline Physical baryon density parameter & $\omega_{b} h^{2}$ & $0.02230 \pm 0.00014$ \\
Physical dark matter density parameter & $\omega_{D M} h^{2}$ & $0.1188 \pm 0.0010$ \\
Reionization optical depth & $\tau$ & $0.066 \pm 0.012$ \\
The age of the universe & $t_{0}$ & $13.799 \pm 0.021 * 10^{9}$ years \\
Scalar spectral index & $n_{S}$ & $0.9667 \pm 0.0040$ \\
Curvature fluctuation amplitude & $\triangle_{R}^{2}$ & $2.441_{-0.092}^{+0.088} * 10^{-9}$ \\
\hline
\end{tabular}

Cold dark matter is a dark matter model. "Cold" indicates that the speed of dark matter is very small compared to the speed of light. "Dark" refers to its weak interactions with photons. The reason of "cold" is that cold dark matter can form early, gravitationally bound clumps. After that, the baryonic matter falls into the gravitational potential of these clumps. So the first stars and galaxies can be formed. Numerical simulations of galaxy formation matches the observation of the $\mathrm{CMB}$ and structure observed by galactic surveys [170].

\subsubsection{Cold dark matter halo}

Cold dark matter halos are the main form into which dark matter collapses. They are hypothetical structures that have decoupled from cosmic expansion and contains matter bounded together by gravitational forces. Dark matter halos played a major role in the process of formation of galaxies at early stage. At that time, the temperature of the baryonic matter was too high so that they cannot form gravitationally self-bound objects. Therefore, additional gravity from prior formation of structure of dark matter is necessary.

The formation of dark matter halos began from small overdensities in early Universe. These overdensities grew linearly due to gravitational instability, increasing in size and amplitude, until they reached a maximum radius, the so-called turnaround radius. Then the gravity dominated over the expansion of the Universes. The overdensities started to collapse and finally virialized. Afterwards, they form gravitationally bound dark matter halos and stopped collapsing. 


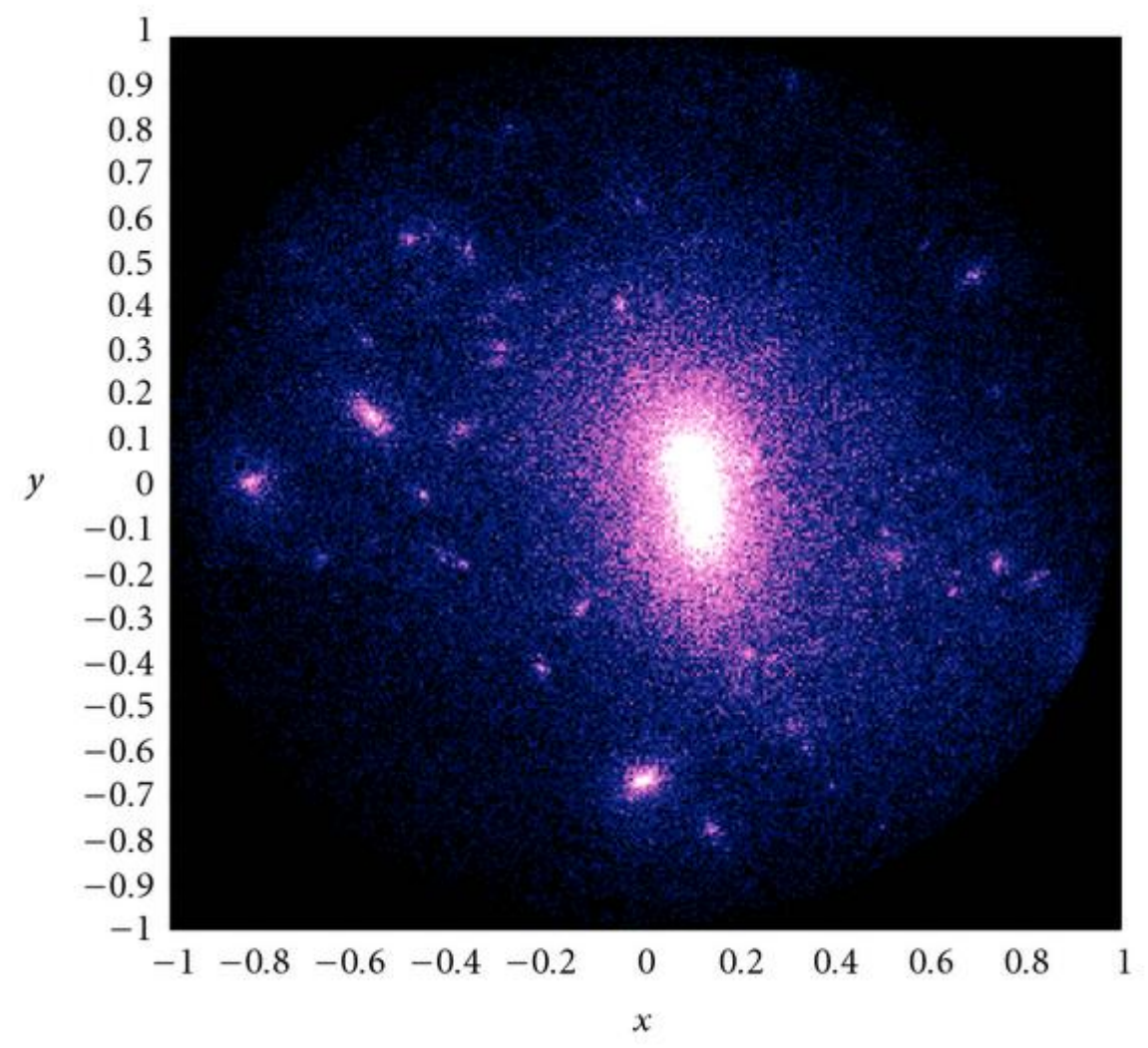

Figure 1.4: Dark matter halo from cosmological N-body simulations. Reproduced from [174]. 
A dark matter halo is a 3-dimensional and nearly spherical object in contrast to 1-dimensional sheet or 2-dimensional filament structure. In this thesis, I will mainly focus on dark matter halos. But it should be noted that dark matter in the Universe form much more complex structure with the halos connected by filaments, the so-called cosmic web.

In cold dark matter model, the dark matter particles are collisionless. A simple solution for a stationary, spherical and isotropic halo is the isothermal model, in which case the halo has a uniform temperature at different radii and the gravity is balanced by the motion of particles. The isothermal model predicts a radial density profile $\propto r^{-2}$. A slightly modified version is the pseudo-isothermal model [74],

$$
\rho(r)=\rho_{0}\left(1+\left(\frac{r}{r_{c}}\right)^{2}\right)^{-1}
$$

where the singular density at the center is replaced by a flat core with density $\rho_{0}$ and radius $r_{c}$. This model has a good fit to many observation data on rotation curves, but in the realistic case a halo may deviate from the isothermal assumption. For example, in the outer areas of dark matter halos, collapse perhaps never reach an equilibrium state.

In contrast, large N-body cosmological simulations with cold dark matter have shown that dark matter halos have a universal density profile, the Navarro-FrenkWhite (NFW) profile [128],

$$
\rho(r)=\frac{\rho_{c}}{\frac{r}{R_{s}}\left(1+\frac{r}{R_{s}}\right)^{2}}
$$

where $\rho_{c}$ and $R_{s}$ are the characteristic density and scale radius of the halo, respectively. Unlike the isothermal model, NFW profile fall off faster at larger radii. The NFW profile is one of the most popular models for dark matter halos since it is suitable for a large range of halo masses, from individual galaxies to whole galaxy clusters.

These results are of great significance to the study of cosmology. In fact, the observation illustrates a larger, approximately spherical dark matter halo embed in the visible disk of the Milky Way Galaxy. And the density of the dark matter drops with distance from the center of Milky Way Galaxy. The total mass of visible matter is roughly $9 \times 10^{10} \mathrm{M}_{\odot}$. On the other hand, the total mass of dark matter is approximately $0.6-3 \times 10^{12} M_{\odot}[86]$. 


\subsection{Candidates particles of cold dark matter}

The model of cold dark matter alone provides very little information about the properties information about the properties of the dark matter particles. We only know it is electromagnetically neutral and it is stable on cosmological time scales. Even its mass remains unknown. Scientists have proposed many potential particles as candidates for dark matter.

\subsubsection{Weakly interacting massive particles}

One popular cold dark matter candidate is the WIMPs. The main theoretical characteristics of a WIMPs are:

- Large mass compared to standard model particles.

- Interacts either only through the gravity and weak nuclear force or the other interaction with cross sections much smaller than that of the weak nuclear force [87].

WIMPs can solve many cosmological and astrophysical problems. For example, they also are well tested on large scales ( $\gtrsim 10 \mathrm{kpc}$ ) by current observations. However, as cold dark matter, WIMPs face a number of potential problems on small scales $(\lesssim 10 \mathrm{kpc})$. These problems include [186]:

- Missing Satellites Problem: the N-body simulations predict that the number of satellite galaxies in the Milky Way from N-body simulations of cold dark matter is significantly more than the observation.

- Cusp-Core Problem, cold dark matter simulations shows that dark matter halos have "cuspy" distributions, which means density increase steeply at small radii. But most of observation of dwarf galaxies find that they have flat central density profiles.

- Too-Big-To-Fail Problem, the most massive dark matter subhalos from cold dark matter simulations are too dense to host the brightest satellite galaxies of the Milky Way.

These problems inspire various alternative models such as warm dark matter and hot dark matter, which can be looked as variants of WIMPs dark matter paradigm. 
The warm dark matter model is one of the popular dark matter solutions for solving small scale problems. Through a cut-off in the matter power and freestreaming, the formation of structure is suppressed on small scales. Therefore, warm dark matter can address the too-big-To-fail problem and missing satellites problem [103]. On the other hand, cores can be formed from warm dark matter due to thermal velocities and Fermion degeneracy pressure [178]. Fig. 1.5 shows that the relationship of core size $r_{c}$ and mass of warm dark matter particle $m_{v}$ given by $r_{c} \sim m_{v}^{-1 / 2}$. However, one problem arises: the core sizes in galaxies is too small when we consider constraints from satellite abundance. For example, in 2012, numerical simulations by Macciò et al. found that when satisfying the constraints from large scale structure formation, the size of core $r_{c} \sim 10(20)$ pc for warm dark matter halo with mass $10^{10} M_{\odot}\left(10^{8} M_{\odot}\right)$. It is much smaller than the size of core from the observation, e.g. $r_{c} \sim \mathrm{kpc}$ in Fornax or Sculptor [104, 185]. This is so the called Catch 22 problem. Similarly, there are problems with hot dark matter models as well. For example, the large-scale structure of galaxies would be smeared out in hot dark matter model [153].

\subsubsection{Ultralight bosons in cosmology}

To solve the small-scale problems mentioned in last section, we introduce ultralight bosons, which could be composed of a scalar field with two parameters: selfinteraction strength and particle mass [119]. As a candidate particle of cold dark matter, light (sub-eV), bosons show new phenomenology on small scales, $<10 \mathrm{kpc}$ but have similar behaviours as WIMPs on large scales, $>10 \mathrm{kpc}$ [116,117]. The deviation from cold dark matter happens at the scale of the virial velocity de Broglie wavelength of a halo. Based on this, particles with a mass of $10^{-22} \mathrm{eV}$ and velocity of $100 \mathrm{~km} / \mathrm{s}$ will present new features on kpc scales [117, 152]. For example, for Fornax dwarf, using Jeans analysis, the mass of ultra-light boson is inferred to be $m_{a}=8.1_{-1.7}^{+1.6} \times 10^{-23} \mathrm{eV}[156]$.

\subsubsection{Axions}

Ultra-light axions is one possible composition of ultra-light bosons. Axions are hypothetical elementary particles postulated in the PQ theory when Roberto Peccei and Helen Quinn tried to solve the strong CP problem in quantum chromodynamics (QCD) in 1977 [135].

There are four production mechanism of axion population: 


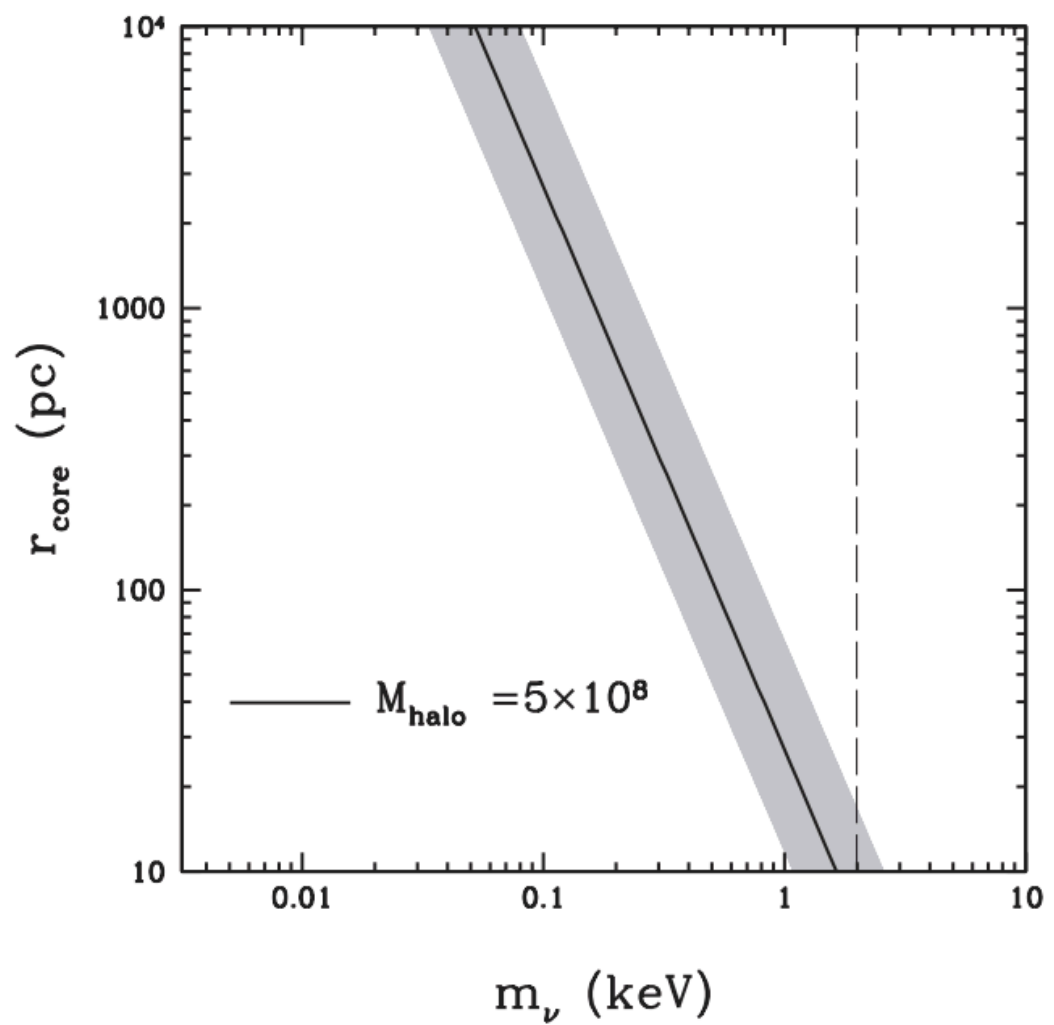

Figure 1.5: The size of core in a warm dark matter halo of mass $M=5 * 10^{8} M_{\odot}$ as a function of warm dark matter thermal relic mass. Reproduced from Ref. [104. 105]. 

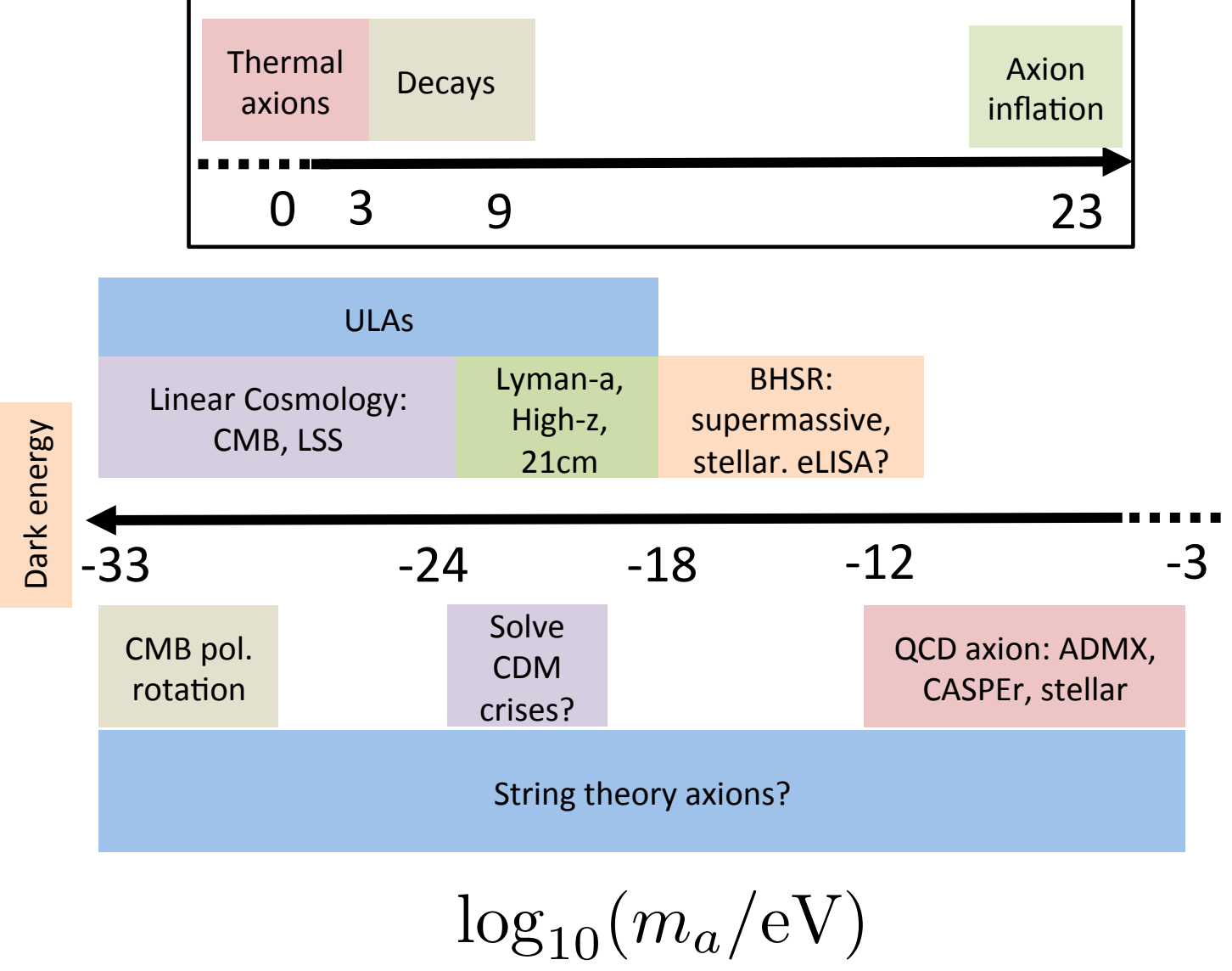

Figure 1.6: Summary of probes and constraints of axion cosmology. Reproduced from Ref. [112].

- Decay product of topological defect;

- Decay product of parent particle;

- Vacuum Realignment;

- Thermal population from the radiation bath.

Depending on the detailed models, the mass of different types axions have different values, see Fig, 1.6 .

Combined with astronomical observations, the axions are required to have a very small mass and either an extremely weak electromagnetic interaction or electromagnetic interaction at all. Theories show that if the mass of axions is very low, our Universe will be filled with Bose-Einstein condensate of axions. This makes axions a very good candidate for dark matter [162]. 


\subsubsection{QCD axions}

As the first axion model, the QCD axion is the historical starting point to the study of axion cosmology. This specific axion is distinct from ultralight bosons. While it is possible to use the quantum effects of the QCD axion in solve the small scale problems of cold dark matter. the relevant mass range $\left(10^{-10}-10^{-4} \mathrm{eV}\right)$ is heavily constrained. However, the QCD axion is a key component of the wider context into which the study of ultralight bosons in embedded. It is therefore worthwhile spending some time introducing this model here.

Within the QCD Lagrangian there exists a natural term which violates the combination of charge conjugation symmetry and parity symmetry.

$$
\mathcal{L}_{\theta \mathrm{QCD}}=\frac{\theta_{\mathrm{QCD}}}{32 \pi^{2}} \operatorname{Tr} G_{\mu \nu} \tilde{G}^{\mu \nu},
$$

where $G_{\mu \nu}$ is the gluon field strength tensor, $\tilde{G}^{\mu \nu}=\epsilon^{\mu v \alpha \beta} G_{\alpha \beta} / 2, \epsilon^{\mu v \alpha \beta}$ is LeviCivita symbol. The term of $\mathcal{L}_{\theta \mathrm{QCD}}$ predicts a non-vanishing neutron electric dipole moment $d_{n}$ when $\theta_{\mathrm{QCD}} \neq 0$ [50],

$$
d_{n} \approx 3.6 \times 10^{-16} \theta_{\mathrm{QCD}} e \mathrm{~cm} .
$$

Naturalness arguments suggest we should expect $\theta_{\mathrm{QCD}} \sim \mathcal{O}(1)$. However, experiments show that $d_{n}$ is extremely small. This "fine tuning" problem is the essence of the strong CP problem. Increasingly precise measurements have constrained $d_{n}$ to be smaller than $2.9 \times 10^{-26} e \mathrm{~cm}$ " [20]. This then gives us a limit on the QCD parameter $\theta_{\mathrm{QCD}}<10^{-10}$.

In 1977, Peccei and Quinn found that the strong CP problem could be solved via the introduction of a global chiral U(1) symmetry. After replacing the parameter $\theta_{\mathrm{QCD}}$ with dynamical field $\varphi$, the Goldstone boson of the spontaneously broken PQ symmetry can be described by

$$
\tilde{\theta}_{\mathrm{QCD}}=\left(-\theta_{\mathrm{QCD}}+\frac{\xi \varphi}{f_{a}}\right) \frac{1}{32 \pi^{2}} \operatorname{Tr} G_{\mu \nu} \tilde{G}^{\mu \nu},
$$

where $f_{a}$ and $\xi$ are the axion decay constant and the colour anomaly respectively. At the minimum of the effective potential $\varphi=\frac{\theta_{\mathrm{QCD}} f_{a}}{\tilde{\zeta}}$, thus making the term of $\mathrm{CP}$ violating be 0 . This then explains the absence of the neutron electric dipole moment and therefore solves the strong CP problem.

It was later realised that doing this also introduces a new particle that we now know as the QCD axion. The mass of QCD axion is given by

$$
m_{a}=6 \times 10^{-6} \mathrm{eV} \frac{10^{12} \mathrm{GeV}}{\mathrm{f}_{\mathrm{a}}}
$$


This equation is independent of the specific model for the QCD axion. When the decay constant $f_{a}$ is large, the QCD axion is both light and stable on cosmological timescales. Therefore, it is a very promising candidate for dark matter.

At present, the most popular models for the $\mathrm{QCD}$ axion are:

- The Peccei-Quinn-Weinberg-Wilczek (PQWW) axion [187, 189];

- The Kim-Shifman-Vainshtein-Zakharov (KSVZ) axion [91, 160];

- The Dine-Fischler-Srednicki-Zhitnitsky (DFSZ) [55, 198].

\subsubsection{Axions in string theory}

String theory needs more than the standard four space-time dimensions. For instance, the critical superstring requires 10 space-time dimensions, and M-theory even needs 11 space-time dimensions [24,73].

We usually need to roll up and make compact the extra dimensions to connect the 10 or 11 dimensional string theory with the 4 dimensional real world. This is process is known as compactification. In order to produce suitable phenomenology including some chiral matter and unbroken SUSY, the compact manifold must be Calabi-Yau [35]. The antisymmetric tensor fields exist in the supergravity characterization of string theory. One possible case is that the antisymmetric partner of the metric exist in all string theories. The Kaluza-Klein (KK) zero modes of antisymmetric tensors of Calabi-Yau lead the emergence of axions [191]. The Hodge numbers of the compact manifold determine the number of axions.

\subsection{Detection of dark matter particles}

As we have seen, there are many astronomical evidence for the existence of dark matter. Therefore, it is highly pressing that we detect this dark matter particle. In fact, attempts to do so are already underway.

One of prediction is that dark matter can recoil off of the nuclei of atoms and emit photons as they pass through the earth. This provides us with a possible method to detect the dark matter. In order to decrease the interference from cosmic rays, such experiments need to operate deep underground to maintain a low background. There already exist several such underground laboratories. These include as Laboratory Nazionali del Gran Sasso(LNGS) [122] (currently the largest 
underground research center in the world), China Jinping Underground Laboratory [194] and Sanford Underground Research Facility [9] as well as many others.

Most of these experiments use noble liquid or cryogenic detector technologies. Cryogenic detectors can detect the heat from particles colliding atoms in a crystal absorber. Noble liquid detectors can detect scintillation produced when particles collide with the nuclei of the liquid of argon or xenon. Both of these techniques focus on distinguishing between background particles and dark matter particles.

In contrast with laboratories built underground, many attempt instead to detect the decay or self-annihilation of dark matter [44, 68, 82]. For example, in areas with high dark matter density, two particles of dark matter can annihilate to produce Standard Model particle-antiparticle pairs or gamma rays [120]. This provides a good way to search for the presence of of dark matter in outer space. Many experiments try to look for such emission. Examples of such experiments include the Fermi Gamma-ray Space Telescope (GLAST) and the Alpha Magnetic Spectrometer(AMS-02) [17]. GLAST is a space observatory constructed to perform gamma-ray astronomy. AMS-02 is a particle physics experiment module installed on the International Space Station [8].

On the other hand, directly producing the dark matter particles is an alternative method to detect dark matter. Large Hadron Collider (LHC), the Large Electron-Positron Collider (LEP) and some other largest particle accelerators can be used to detect possible dark matter particles.

\subsubsection{Detection of Axion dark matter}

Significant efforts are also being made to detect axion dark matter. Two of the main types of direct detection experiments employed are haloscopes and helioscopes [16]. Haloscopes attempt to detect the axions which make up the dark matter in our local neighbourhood, while helioscopes attempt to detect axions that have been produced in stars, namely the Sun, via the Primakoff effect. Both of these types of experiment primarily measure the axions coupling to photons $g_{\phi \gamma}$, however experiments measuring the couplings to other SM particles also exist.

One of the most famous haloscopes is the Axion Dark Matter eXperiment (ADMX) [16]. The main mechanism is to detect photons converted from axions in a microwave cavity. The power conversion of cavity is

$$
P=g_{\phi \gamma}^{2} \frac{V B_{0} \rho_{a} C}{m_{a}} \min \left(Q, Q_{a}\right),
$$


where $\rho_{a}$ is the local density of axions, $Q_{a}$ is the ratio of spread axion energy to energy, $B_{0}$ is the strength of magnetic field and $V, Q$ and $C$ are the volume, quality factor and the mode dependent form factors of cavity respectively. As an axion dark matter detector, ADMX requires a large local density of axion dark matter, $\rho_{a}$. ADMX achieves its high sensitivities by tuning their cavity to be in resonance with specific axion masses. However, this makes constraining a wide range of masses very slow and so far ADMX has only been able to rule out a comparatively small range in the mass-coupling plane, $\mathrm{QCD}$ axion with $10^{-6} \mathrm{eV} \lesssim \mathrm{m}_{\mathrm{a}} \lesssim 10^{-5} \mathrm{eV}$, i.e. decay constant $f_{a} \sim 10^{12} \mathrm{GeV}$.

In response to this situation, other haloscopes have been proposed including the ORPHEUS experiment [151], MADMAX [110] and TOORAD [114] to name a few. There are also experiments proposing the use of novel technologies such as SQUIDs [115], and LC-circuits [163]. Additionally, ADMX have plans to extend their current constraints [16]. These experiments all try to detect QCD axion dark matter via the two-photon coupling. A summary of some of the experiments mentioned are presented in Fig. 1.7. Combined, these experiments are ability to detect the $\mathrm{QCD}$ axions with wider mass range, $10^{-8} \mathrm{eV} \lesssim \mathrm{m}_{\mathrm{a}} \lesssim 10^{-2} \mathrm{eV}$.

One other particularly interesting proposed haloscope is the Cosmic Axion Spin Precession Experiment (CASPEr) [32], which employ two new strategies. These experiments don't depend on the "standard" two-photon coupling. The mechanism CASPEr experiments will measure the spin dependence of the coupling between axions and nucleons. Through spin-polarizing samples in the applied magnetic field and looking for spin-precession by techniques of nuclear magnetic resonance, we can detect the interactions.

CASPEr-Electric exploits the coupling of axions and $\left(\phi / f_{a}\right) \operatorname{Tr} G \tilde{G}$, which promote the electric dipole moment (EDM) coupling. Therefore, CASPEr-Electric explores the defining characters of QCD axions. In phase 2 CASPEr-Electric can detect the QCD axion with $f_{a} \gtrsim 10^{16} \mathrm{GeV}$.

On the other hand, as our Earth moves through the dark matter halo, the dark matter "wind" can pass through the Earth. This also provide us a good method to search axions. CASPEr-Wind can detect the coupling of the axial nuclear current and axions, or the coupling of the induced spin-dependent force and axions at this time. The projected sensitivities of CASPEr-Wind show that it will not be sensitive to the $\mathrm{QCD}$ axions but, more interestingly for us, may be able to detect ultra-light axions. 


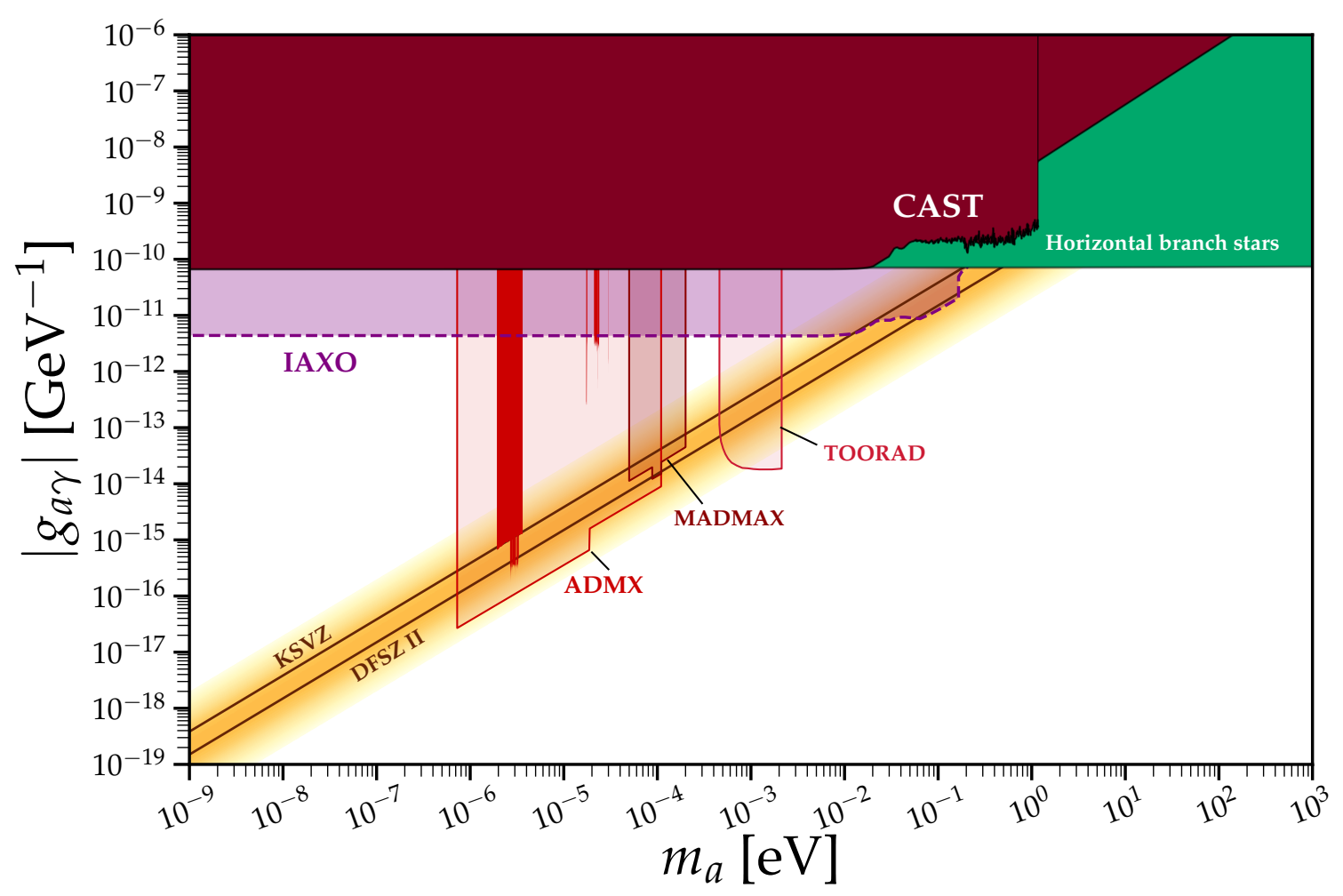

Figure 1.7: A small sample of some of the existing (solid) and proposed (transparent) experimental constraints on the axion-photon coupling. The yellow band indicates the range of parameter space in which different models of the QCD axion predict the particle to be. Produced using the public code provided by Ref. [132] 
Unlike most haloscopes, helioscopes are broadband searches and therefore apply constraints on the ultra-light axions we are interested in. To date, the most sensitive helioscope is the CERN Axion Space Telescope (CAST) [12, 14, 22]. This experiment uses a 9T refurbished test magnet from the LHC to try to convert axions produced in the sun into detectable photons. For axion masses $m_{a} \lesssim 10^{-2} \mathrm{eV}$, CAST constrains the axion-photon coupling to $g_{a \gamma} \lesssim 0.66 \times 10^{-10} \mathrm{GeV}$. This only just about reaches the constraints derived from considering the cooling of horizontal branch stars [76]. However, these constraints will soon by surpassed by the International AXion Observatory (IAXO) as shown in Fig 1.7 .

\subsubsection{Thesis Overview}

In this dissertation, we start in Chapter. 2 with mainly introducing boson stars and our numerical methods. In Sec. 2.1. we show some properties of boson stars. In Sec. 2.2, we introduce the solutions of boson stars of Gross-Pitaevskii-Poisson (GPP) equations. After that, we introduce the relationship between boson stars and dark matter halos in Sec. 2.4. Our numerical method and simulations are introduced in Sec 2.5 and Sec 2.6 .3

Chapter. 3 studies the formation and evolution of boson stars and halos. We firstly introduce theory of condensation of bosons in Sec. 3.1. In Sec. 3.2.3 we study the formation and saturation of boson stars for bosons without self-interaction. In Sec. 3.3.1 and Sec. 3.3.2 we study the evolution of bosons with both attractive and repulsive self-interactions. In Sec 3.4, the strong attractive self interaction has been considered. This chapter primarily is based on our publication [41].

The study of turbulence creation in the merger of boson stars is outlined in Chapter.4. In Sec. 4.1. I give a general introduction to the mechanism of quantum turbulence. After that, I perform numerical simulations of the mergers of multiple boson stars and analyze the turbulence creation and their properties in systems, see Sec. 4.2 .

In Chapter 5. I summarize my dissertation, and provide an outlook about the extension of my current work. 


\section{Chapter 2}

\section{Boson stars and numerical methods}

\subsection{Boson stars}

Boson stars are hypothetical astronomical objects which are composed of scalar particles in their ground state. For this type of star to form, there must exist a stable boson with self-repulsive force that balances the gravity [96]. Ultra-light bosons are good candidates due to the quantum pressure. Since ultra-light bosons are prominent dark matter candidates, understanding the formation and evolution of these boson stars may be the key to understanding the nature of dark matter.

\subsubsection{Compact boson stars}

In 1967, by solving the coupled Einstein-Klein-Gordon equations, David J. Kaup demonstrated the possible existence of compact boson stars theoretically [88]. The dynamical Einstein-Klein-Gordon equation equations can be derived from the action,

$$
\mathcal{L}_{B S}=\sqrt{\operatorname{det}\left(g_{\mu v}\right)}\left(\frac{16 \pi G}{R}+\mathcal{L}_{M}\right)
$$

where $g_{\mu v}$ is metric tensor, $G$ is gravity constant, $R$ is $g^{\mu v} R_{\mu v}, R_{\mu v}$ Ricci tensor, and natural units, $\hbar=c=1$, are used. The matter is represented by $\mathcal{S}_{M}$. For a complex scalar field, $\phi$, we have

$$
\mathcal{L}_{M}=-\frac{1}{2} g^{\mu v} \partial_{\mu} \phi^{*} \partial_{v} \phi-V\left(|\phi|^{2}\right),
$$

where $\phi^{*}$ is the complex conjugate of $\phi$ and $V\left(|\phi|^{2}\right)$ is the potential of the scalar field. The metric tensor, $g_{\mu v}$ can be solved via the Einstein equations,

$$
R_{\mu v}-\frac{1}{2} g_{\mu v} R=-8 \pi G T_{\mu v}(\phi),
$$


and the stress-energy tensor

$$
T_{\mu v}=\frac{1}{2}\left(\partial_{\mu} \phi^{*} \partial_{v} \phi+\partial_{\mu} \phi \partial_{v} \phi^{*}\right)-\frac{1}{2} g_{\mu v}\left(g^{\alpha \beta} \partial_{\alpha} \phi^{*} \partial_{\beta} \phi+V\left(|\phi|^{2}\right)\right) .
$$

Boson stars may have condensed due to gravity during the primordial stages of the Big Bang [107]. The solution of $\phi$ shows that the strong gravity of a compact boson star could significantly bend the light passing by. This creates an empty region similar to the shadow of the event horizon of a black hole. On the other hand, compact boson stars would be transparent and invisible because there is no interaction between boson star and photons other than gravity. However, a compact boson star, as a invisible celestial object, would attract ordinary matter from its surroundings [121].

Some astronomical observations provide support for the existence of such objects. For instance, several observations imply the existence of a supermassive compact dark object at the center of Sgr A* [69]. Some have claimed that this supermassive compact dark object a supermassive black hole. However, it has been shown that if the compact object is a supermassive black hole, its luminosity would exceed $10^{40} \mathrm{erg} \mathrm{s}^{-1}$. However, after correcting the attenuation of gas and dust, observations find the luminosity to be less than $10^{37} \mathrm{erg} \mathrm{s}^{-1}$. Obviously, observations deviates from predictions. This is known as the 'blackness problem'. In contrast, the dynamical data and observed luminosity of the center of Sgr A* can be explained by the existence of a massive boson star [177, 183].

In addition, it is extremely difficult to observe low-brightness astrophysical compact objects by current electromagnetic telescopes. But gravitational wave astronomy allows us to detect these stars in Universe. The analysis indicates that the gravitational signature from merger of compact boson stars can be distinguished from other astrophysical objects such as black holes and neutron stars [27]. This provides a good way for us to search for boson stars as well as dark matter in the future.

\subsubsection{Non-compact boson stars}

Solving Einstein equation is always difficult. But for bosons in the non-relativistic limit, i.e. low density and low velocity, the metric tensor of weak gravity field can be approximated as

$$
g_{00}=-1+2 V, g_{i i}=1-2 V, g_{i j}=0(i \neq j) .
$$


Therefore, the metric tensor can be written as

$$
d s^{2}=-(1+2 V(\vec{x}, t)) d t^{2}+(1-2 V(\vec{x}, t))\left(d x^{2}+d y^{2}+d z^{2}\right) .
$$

Ultralight bosons can be described by a classical scalar field $\phi$, as discussed at length in the literature [3, 56, 75, 144, 172, 180, 188]. The potential of the scalar field can be expanded for small field values as

$$
V(\phi)=\frac{1}{2} m^{2} \phi^{2} \pm \frac{1}{4 !} \frac{m^{2}}{f_{a}^{2}} \phi^{4}+\ldots,
$$

where $m$ is the particle mass, $f_{a}$ is the decay constant, and natural units, $\hbar=c=1$, are used. The plus-minus sign before the $\phi^{4}$ term corresponds to repulsive ( + ) and attractive (-) self-interaction, respectively. In this paper, we define the dimensional self-coupling constant as $g \equiv \pm \frac{1}{8 f_{a}^{2}}$. [15, 98, 164].

If the bosons are very light, the occupation number must be very high to make up all of the dark matter in the Universe. Therefore, we can describe bosons as a classical field. The action of the classical field is given by

$$
\mathcal{S}=\int \mathcal{L}\left(\phi, \partial_{\mu} \phi\right) d^{4} x=\int \sqrt{\operatorname{det}\left(g_{\mu v}\right)}\left[\frac{1}{2}(\partial \phi)^{2}-\frac{1}{2} m^{2} \phi^{2}-\frac{1}{2} m^{2} g \phi^{4}\right] d^{4} x .
$$

Minimizing this action, we derive the Klein-Gordon equation

$$
\ddot{\phi}^{2}-m^{2} \phi-2 m^{2} g \phi^{3}=0
$$

In the non-relativistic limit we can rewrite the scalar field $\phi$ as

$$
\phi=\sqrt{\frac{2}{m}} \operatorname{Re}\left(\psi e^{-i m t}\right) .
$$

Combining the Eqs. 2.9, 2.6 and 2.10, we find that the complex wave function $\psi$ satisfies Gross-Pitaevskii-Poisson (GPP) equations, [39,59]

$$
\begin{array}{r}
i \frac{\partial}{\partial t} \psi=-\frac{1}{2 m} \nabla^{2} \psi+m V \psi+g|\psi|^{2} \psi, \\
\nabla^{2} V=4 \pi G m\left(|\psi|^{2}-n\right) .
\end{array}
$$

One peculiar feature of ultralight bosons is that they exhibit properties similar to that of a superfluid. To understand this, we can use Madulung transformation to convert the GPP equations into the Madelung equation,

$$
\begin{gathered}
\dot{\rho}+\nabla(\rho \mathbf{v})=0, \\
\dot{\mathbf{v}}+\mathbf{v} \nabla \mathbf{v}=-\nabla V+\frac{1}{2 m^{2}} \nabla\left(\frac{\nabla^{2} \sqrt{\rho}}{\sqrt{\rho}}\right)-\frac{g}{\rho} \nabla \frac{\rho^{2}}{2}, \\
\nabla^{2} V=4 \pi G m\left(\rho^{2}-n\right),
\end{gathered}
$$


where we set $\psi=\sqrt{\frac{\rho}{m}} e^{i S}$, and $\mathbf{v}=\nabla S / m$. The Madelung equations are written in terms of hydrodynamical variables, similar to the Navier-Stokes equations of fluid dynamics. The second term on the right hand side of Eq.2.1.2 can be interpreted as a quantum pressure.

For some bosons, such as fuzzy dark matter, the self-interaction is very weak compared to gravity, thus we can neglect the term of $g|\psi|^{2} \psi$, and GPP equation can be approximated by Schrödinger-Poisson (SP) equations, [39, 59]

$$
\begin{aligned}
i \frac{\partial}{\partial t} \psi & =-\frac{1}{2 m} \nabla^{2} \psi+m V \psi, \\
\nabla^{2} V & =4 \pi G m\left(|\psi|^{2}-n\right),
\end{aligned}
$$

Similarly, when self-interaction dominate systems, the GPP equation can be simplified to give the Gross-Pitaevski (GP) equation equations,

$$
i \frac{\partial}{\partial t} \psi=-\frac{1}{2 m} \nabla^{2} \psi+m V \psi+g|\psi|^{2} \psi
$$

Solving the time-independent SP, GP or GPP equations, we find that the finite energy ground state solution of these systems is a soliton: a localized lump of boson energy density held together by the competing forces of gravity, selfinteractions, and gradient energy [81, 113, 158, 188]. Such solitonic solutions are also known as non-compact boson stars.

To date, no analytical solution of solitions has been found. Therefore, to study solitions, we must employ numerical methods. In numerical simulations, we rewrite Eq. 2.11 in a dimensionless form by making the substitutions $x=\widetilde{x} /\left(m v_{0}\right), t=$ $\tilde{t} /\left(m v_{0}^{2}\right), V=\widetilde{V} v_{0}^{2}$ and $\psi=\widetilde{\psi} v_{0}^{2} \sqrt{m /(4 \pi G)}, g=\widetilde{g} 4 \pi G / v_{0}^{2}$, as given by Ref. [99], where $v_{0}$ is a reference velocity, e.g. the characteristic velocity of the initial state. Thus the dimensionless equations are given by

$$
\begin{aligned}
i \frac{\partial}{\partial \widetilde{t}} \widetilde{\psi} & =-\frac{1}{2} \widetilde{\nabla}^{2} \widetilde{\psi}+\widetilde{V} \widetilde{\psi}+\widetilde{g}|\widetilde{\psi}|^{2} \widetilde{\psi}, \\
\widetilde{\nabla}^{2} \widetilde{V} & =|\widetilde{\psi}|^{2}-\widetilde{n},
\end{aligned}
$$

\subsection{Soliton solutions to the GPP equations}

Boson stars with self-interaction exhibit different phenomena to those without [100]. For example, a strong attractive self-interaction can overcome the quantum pressure and lead to the collapse of boson stars. Alternatively, repulsive self-interaction 
can make the radius of boson star larger [108]. Here, we obtain numerical solutions of boson stars with attractive and repulsive self-interaction by solving timeindependent GPP equations and give their best-fitting function.

Our ansatz for stationary solution is given by

$$
\psi(\mathbf{r}, t)=\psi(\mathbf{r}) e^{-i E t} .
$$

Substituting this into Eq. 2.11, we obtain the time-independent GPP equations.

$$
\begin{aligned}
-\frac{1}{2} \nabla^{2} \psi(\mathbf{r}) & =\left[E-V(\mathbf{r})-g|\psi(\mathbf{r})|^{2}\right] \psi(\mathbf{r}), \\
\nabla^{2} V(\mathbf{r}) & =|\psi(\mathbf{r})|^{2} .
\end{aligned}
$$

Here we have written the equations in dimensionless form as Eq.2.14 and 2.15 and dropped the tildes over the dimensionless quantities for simplicity. The soliton solution is the eigenstate of Eqs. 2.17 and 2.18 with the lowest eigenenergy under the boundary conditions

$$
\begin{aligned}
\psi(0) & =\psi_{0} \\
\psi^{\prime}(0) & =0 \\
\psi(\infty) & =0 \\
V^{\prime}(0) & =0 \\
V(\infty) & =0 .
\end{aligned}
$$

In practice, Eqs. (2.17) and (2.18) can be solved numerically using the shooting method. To do this we first let $V(0)=V_{0}, E=E_{0}$ and integrate the equations outward from $r=0$; Next, we adjust the values of $V_{0}$ and $E_{0}$ and repeat the integration until the boundary conditions given by Eqs. (2.21) and (2.23) are satisfied.

It should be noted that the GPP equations have the following scaling symmetry

$$
\{\mathbf{r}, t, \psi, E, V, g\} \rightarrow\left\{\lambda^{-1} \mathbf{r}, \lambda^{-2} t, \lambda^{2} \psi, \lambda^{2} E, \lambda^{2} V, \lambda^{-2} g\right\},
$$

where $\lambda$ is an arbitrary none-zero parameter. Using this scaling symmetry, we can transform one soliton solution to another solution that has a different central density $\rho_{0}=\left|\psi_{0}\right|^{2}$ but the same $g^{2} \rho_{0}$.

For a scalar field without self-interaction, i.e. $g=0$, it has been shown that the density profile of a soliton can be well fit by [113, 155]

$$
\rho_{\text {soliton }}(r)=\rho_{0}\left[1+0.091\left(\frac{r}{r_{\text {core }}}\right)^{2}\right]^{-8},
$$


where $\rho_{0}$ is the central density and core radius $r_{\text {core }}$ is defined as the radius where the density drops to half of the central density, $r_{\text {core }}=1.308 \rho_{0}^{-1 / 4}$. As such only one of the parameters, $\rho_{0}$ or $r_{\text {core, }}$ is required to determine the core profile

However, when the self-interaction is non-negligible, we will need an additional parameter $g^{2} \rho_{0}$ to determine the soliton profile. As $g^{2} \rho_{0}$ approaches 0 , we expect that the soliton has the same density profile as Eq. 2.25. Therefore, assume in the general case the soliton density profile has a form of

$$
\rho_{\text {soliton }}(r)=\rho_{0}\left[1+\left(-1+2^{1 / \beta}\right)\left(\frac{r}{r_{\text {core }}}\right)^{\alpha}\right]^{-\beta},
$$

where $\alpha$ and $\beta$ are functions of $g^{2} \rho_{0}$ only. When $g \rho_{0}^{2} \rightarrow 0$, we require that $\alpha \rightarrow 2$, and $\beta \rightarrow 8$.

We first consider the case with attractive self-interactions, i.e. $g<0$. As is well known, there exists a critical mass above which a boson star with attractive selfinteractions is unstable [38, 39]. In Fig. 2.1, we show the total mass of the boson star, $\sqrt{|g|} M_{\text {total }}$, with respect to its central density, $g^{2} \rho_{0}$. As expected, $\sqrt{|g|} M_{\text {total }}$ increases with $g^{2} \rho_{0}$ and reached a maximum value, 12.72 , at $g^{2} \rho_{0}=0.52$. When the central density increases further, the soliton solution becomes unstable and its total mass decreases as the central density increases.

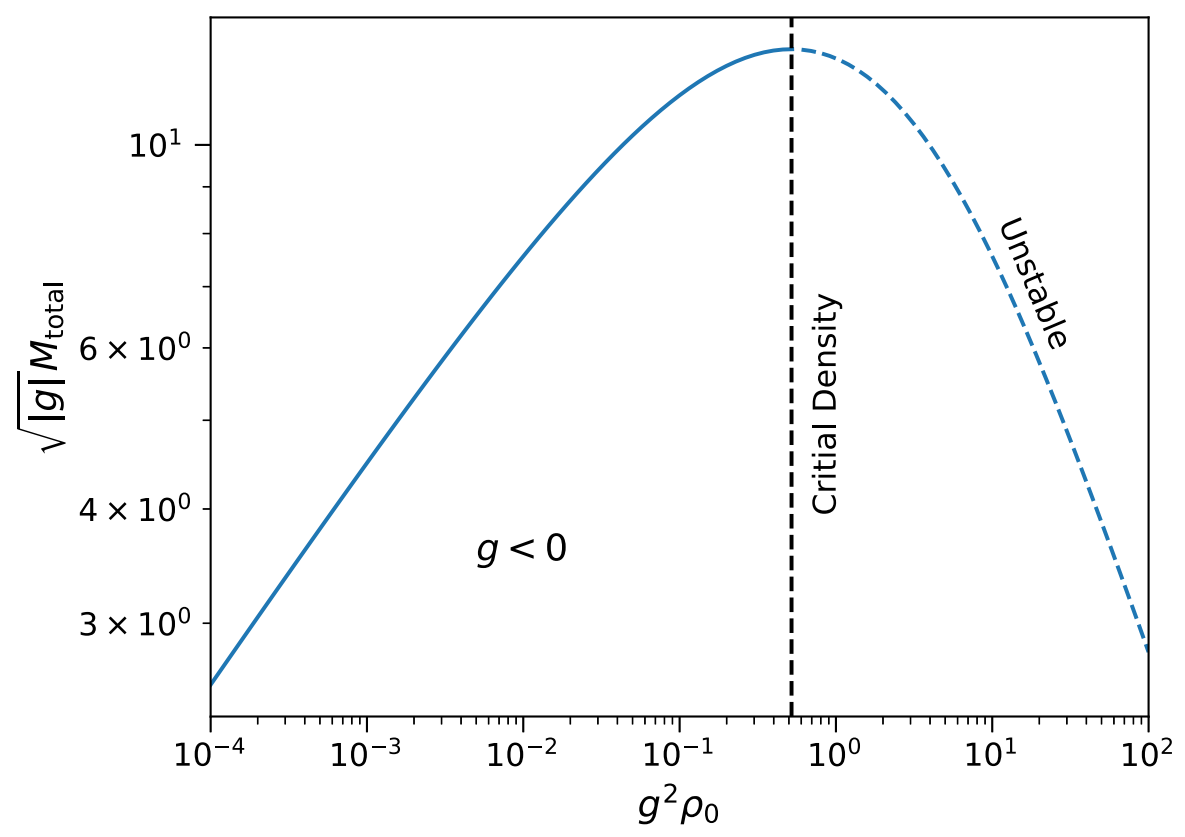

Figure 2.1: Total mass of boson star with attractive self-interactions $(g<0)$ as a function of the central density. 
In order to obtain the fitting formula for the density profile, we also need to know how the core radius depends on $g$ and $\rho_{0}$. Figure 2.2 shows the core radii of boson stars with different central densities. As can been seen, when $g^{2} \rho_{0} \ll 1$, the core radius $r_{\text {core }} \propto \rho_{0}^{-1 / 4}$, recovering the relation seen in the case without selfinteractions. When $g^{2} \rho_{0} \gg 1, r_{\text {core }} \propto \rho_{0}^{-1 / 2}$. Therefore, we assume the core radius has the form

$$
r_{\text {core }}=1.308 \sqrt{|g|}\left[-\frac{a}{2}+\sqrt{\left(\frac{a}{2}\right)^{2}+\frac{1}{g^{2} \rho_{0}}}\right]^{1 / 2},
$$

where $a$ is a free parameter needed which we determine by fitting the numerical results. We find the best-fit value of $a$ is 1.375. Note that the solution with $g^{2} \rho>0.52$ is unstable as discussed previously, but we include all the solutions with $10^{-4}<g^{2} \rho_{0}<10^{2}$ in the fitting process so that we can correctly get the transition between two limits: $r_{\text {core }} \propto \rho_{0}^{-1 / 4}$ and $r_{\text {core }} \propto \rho_{0}^{-1 / 2}$.

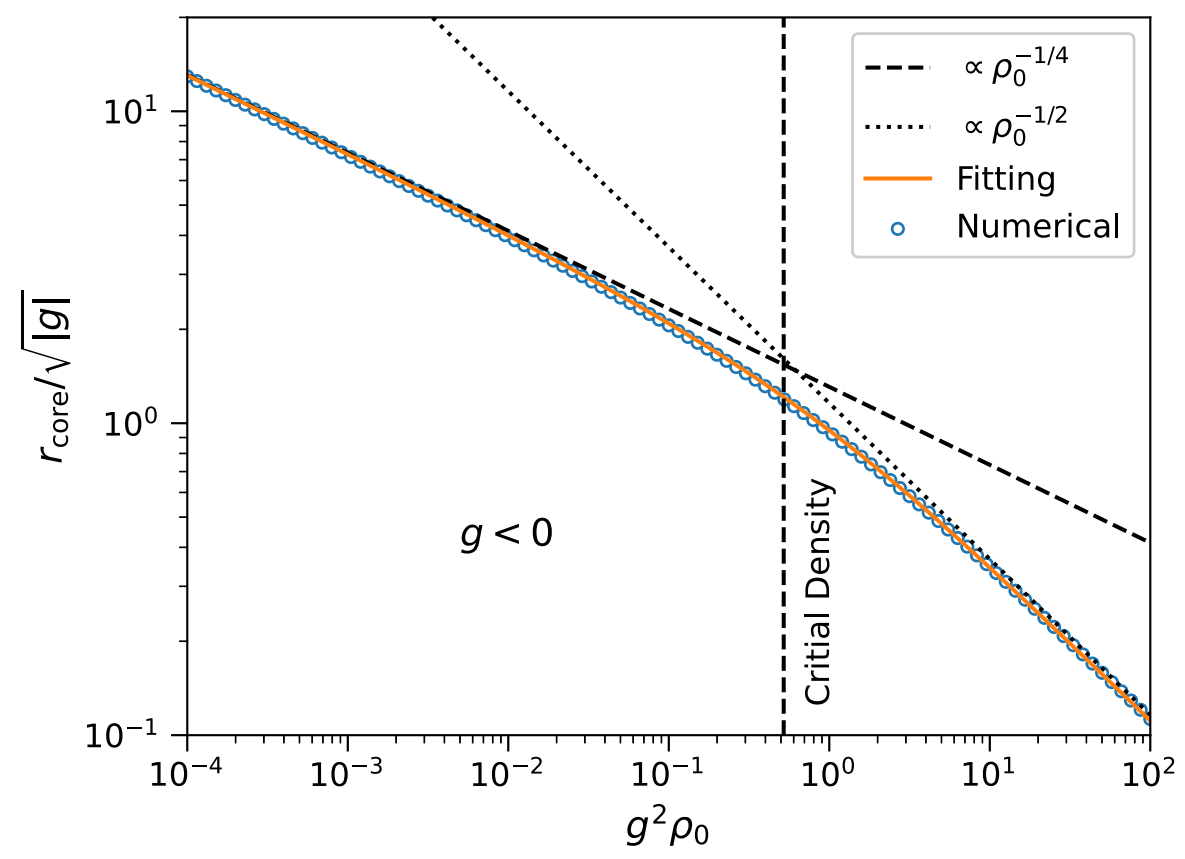

Figure 2.2: Core radius of boson star with attractive self-interactions $(g<0)$ as a function of the central density. Circles: numeric results. Solid line: fitting function, Eq.2.27

To determine the parameter $\alpha$ and $\beta$ for each solution, we first fix the core radius using Eq. 2.27. Then we fit $r^{2} \rho(r)$ within the range $0.01 r_{\text {core }}<r<5 r_{\text {core }}$. The bestfit $\alpha$ and $\beta$ for different $g^{2} \rho_{0}$ are shown in Figs. 2.3 and 2.4 respectively. We find 
that the dependence of $\alpha$ and $\beta$ on $g^{2} \rho_{0}$ can be well fit by

$$
\begin{aligned}
\alpha & =\alpha_{a}+\left(2-\alpha_{a}\right) \tanh ^{8}\left[\alpha_{b}\left(g^{2} \rho_{0}\right)^{-\alpha_{c}}\right], \\
\beta & =\beta_{a}+\left(8-\beta_{a}\right) \tanh ^{8}\left[\beta_{b}\left(g^{2} \rho_{0}\right)^{-\beta_{c}}\right] .
\end{aligned}
$$

The best-fit values for $\alpha_{i}$ and $\beta_{i}(i=a, b, c)$ are listed in Table 2.1.

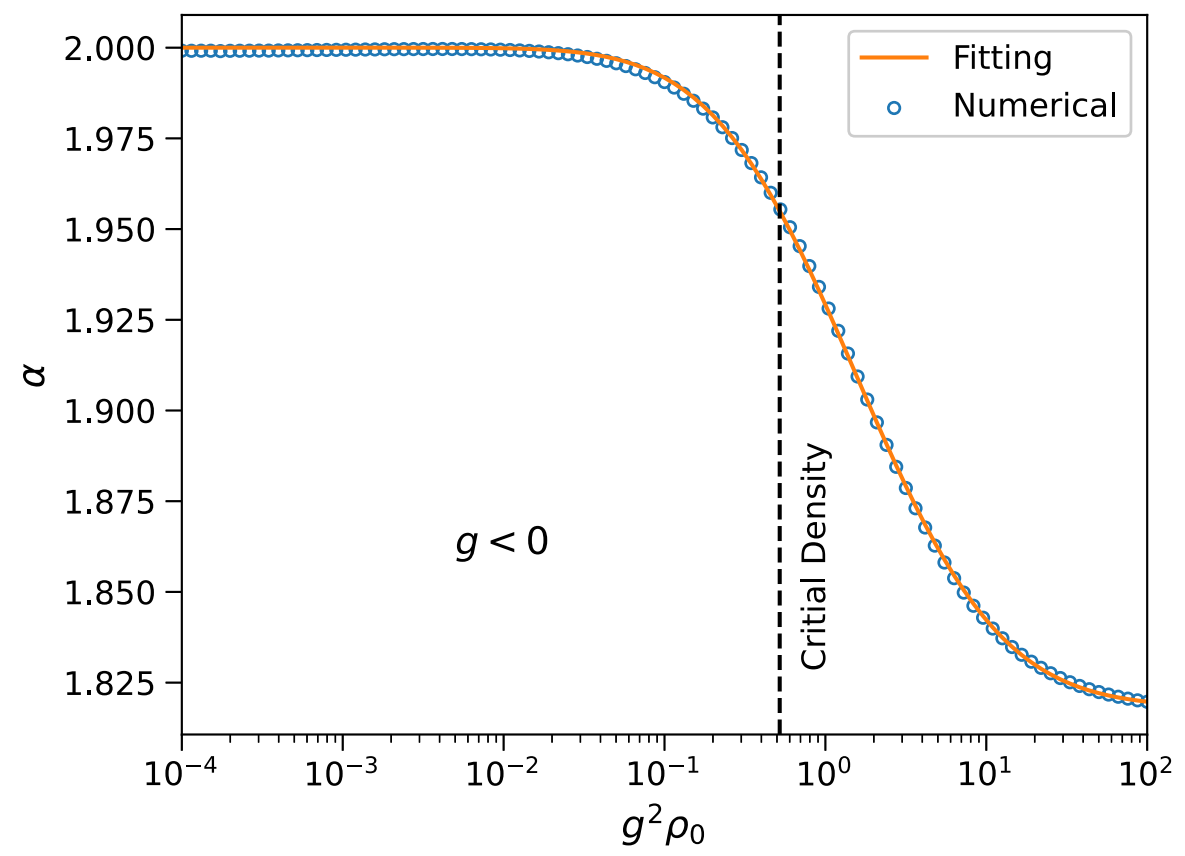

Figure 2.3: Parameter $\alpha$ in the fitting formula Eq. 2.26 for the case with attractive self-interactions $(g<0)$. Circles: numeric results. Solid line: fitting function, Eq. 2.28.

Similarly, we can find the relation between $r_{\text {core }}$ and $\rho_{0}$ for the case with repulsive self-interactions $(g>0)$. We assume

$$
r_{\text {core }}=\left\{\begin{aligned}
1.308 \sqrt{|g|}\left[2+\left(g^{2} \rho_{0}\right)^{-b}\right]^{\frac{1}{4 b}}, & g^{2} \rho_{0} \leq 1.5 \\
c, & g^{2} \rho_{0}>1.5
\end{aligned}\right.
$$

considering that when $g^{2} \rho \gg 1$, we have Thomas-Fermi-like solution at small radii

$$
\rho(r)=\rho_{0} \frac{\sin r}{r},
$$

which gives a constant core radius. We find the best-fit $b=0.710752$, and $c=$ 1.86543. Fig. 2.5 shows the fitting formula of $r_{\text {core }}$ (solid line) compared with the numerical results (circles). 


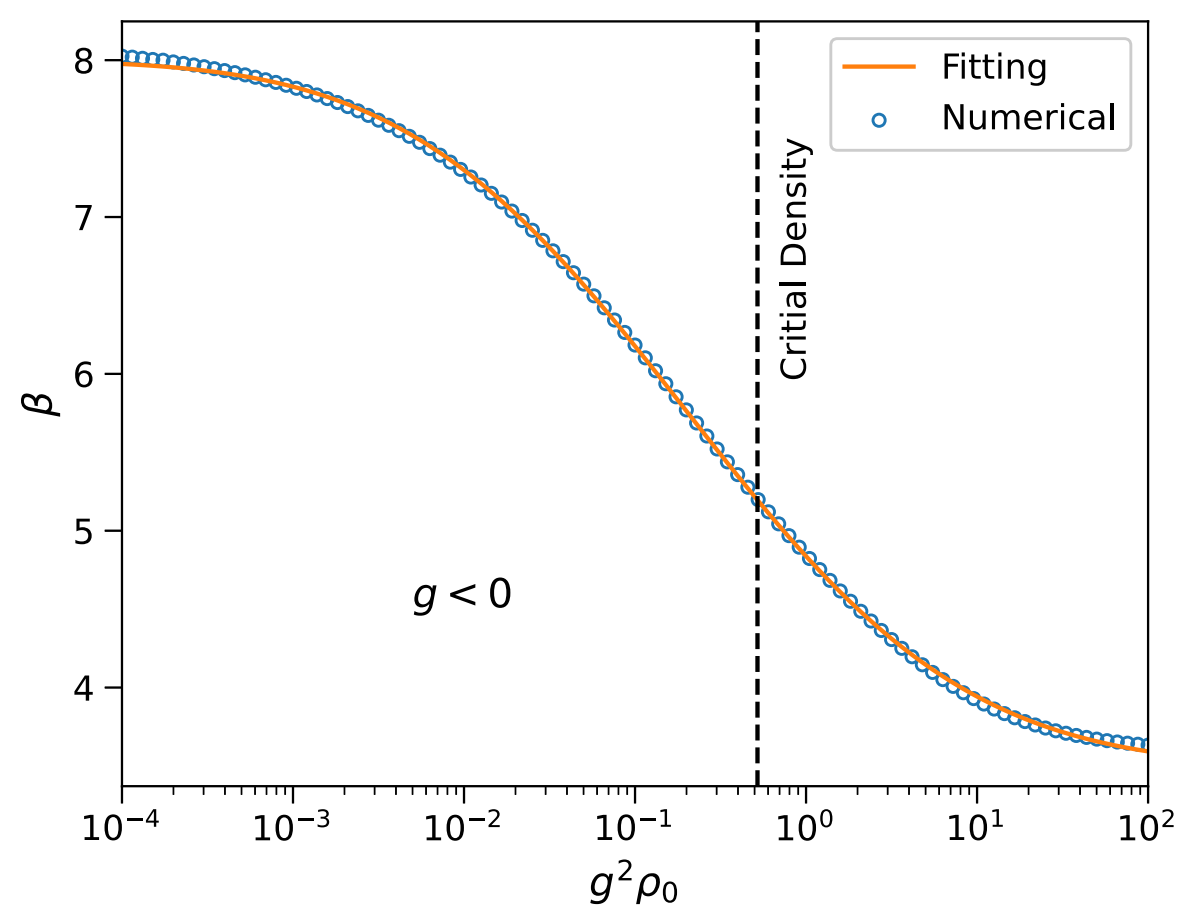

Figure 2.4: Parameter $\beta$ in the fitting formula Eq. 2.26 for the case with attractive interactions $(g<0)$ as a function of the central density. Solid line: fitting function, Eq. 2.29

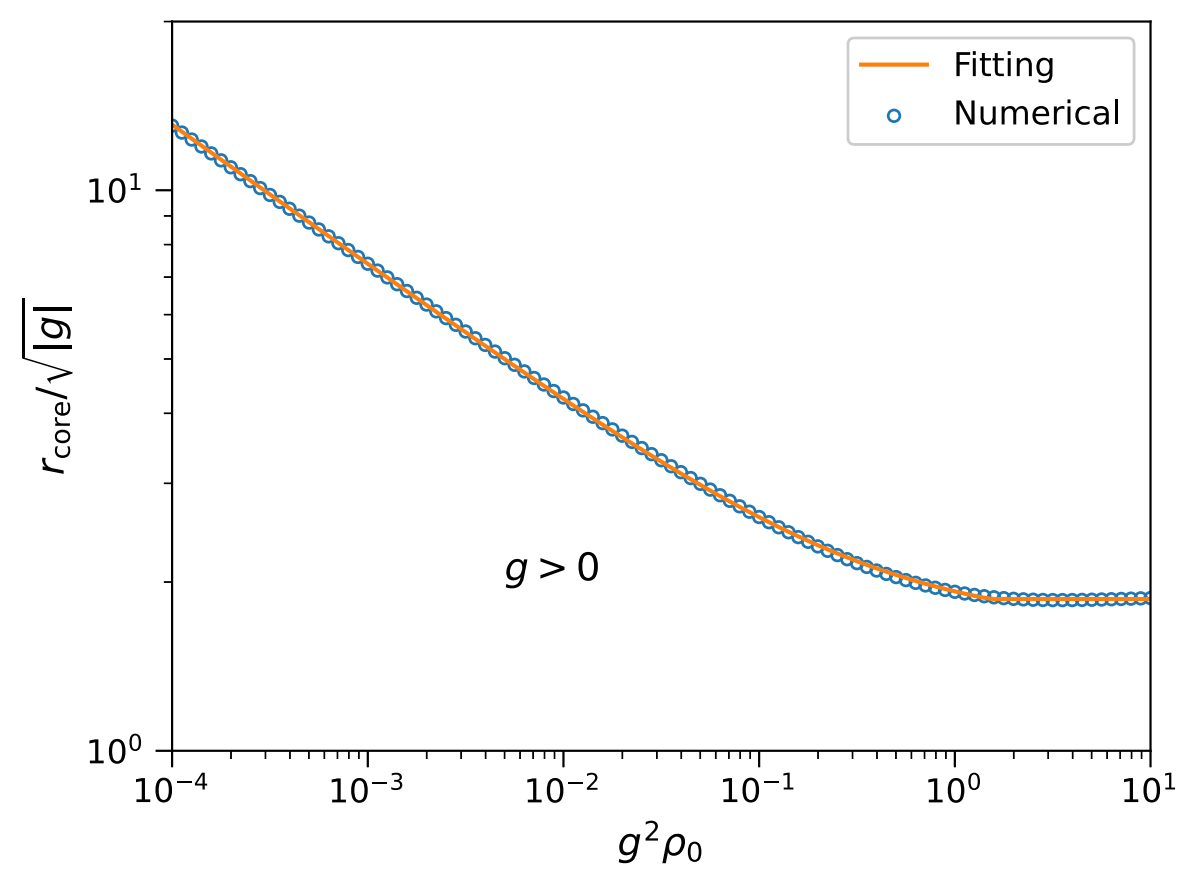

Figure 2.5: Core radius of boson star with repulsive self-interactions $(g>0)$ as a function of the central density. Circles: numeric results. Solid line: fitting function, Eq. 2.30 
As in the $g<0$ case, we also fit $r^{2} \rho(r)$ within the range $0.01 r_{\text {core }}<r<5 r_{\text {core }}$ to the the results obtained from numerical wave function. However, here we have fixed $\beta$ at 8 as allowing $\beta$ to be a free parameter only marginally improves the fit. For the dependence of $\alpha$ on $g^{2} \rho$ we take the same form as in Eq. 2.28. The best-fit values $\alpha_{i}$ are listed in Table 2.1. A comparison between the fitting function of $\alpha$ and the one obtained from numerical results is shown in Fig. 2.6 We have fitted the soliton density for $10^{-4}<g^{2} \rho<10$, but we note that Eq. 2.26 can not well describe the soliton density at very small radii when $g^{2} \rho \gtrsim 1$. In those cases, the Thomas-Fermi-like solution Eq. 2.31 is more accurate at $r<r_{\text {core. }}$

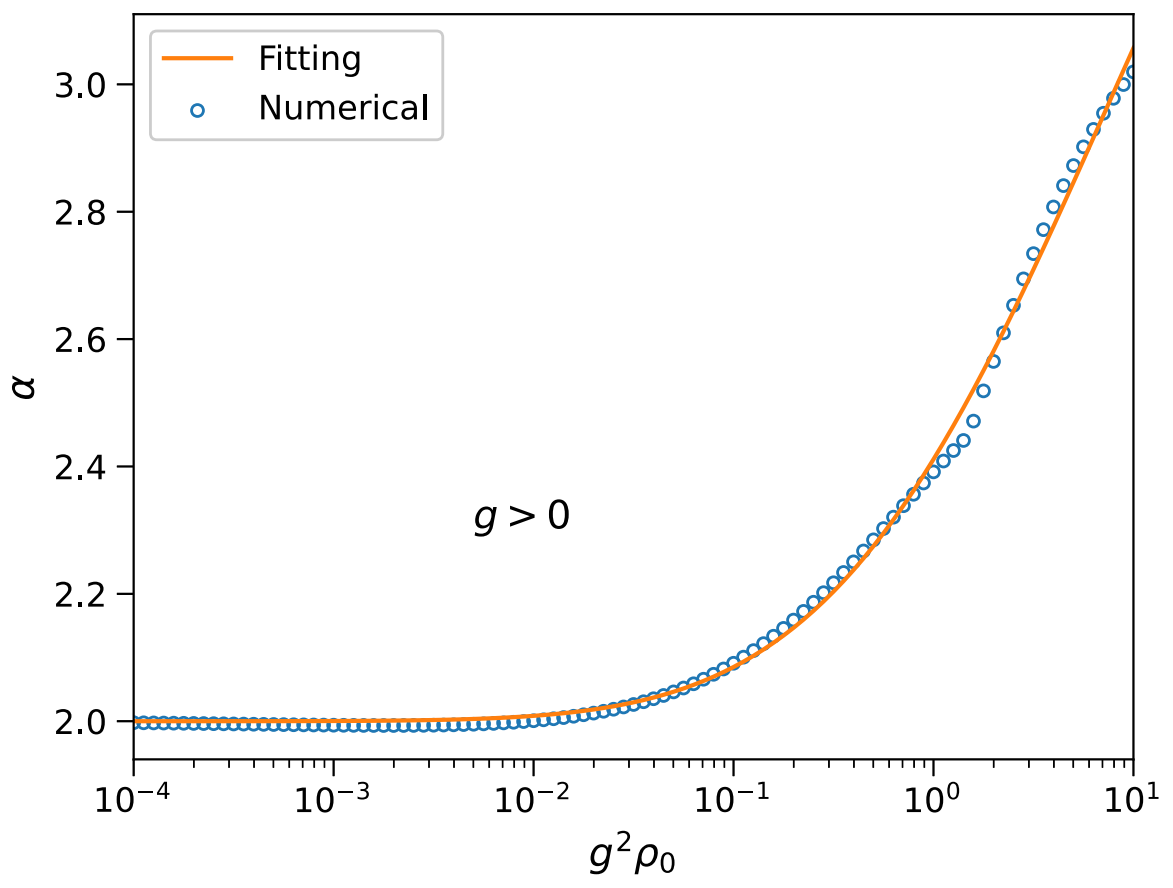

Figure 2.6: Parameter $\alpha$ in the fitting formula Eq. 2.26 for the case with repulsive self-interactions $(g>0)$ as a function of the central density. Circles: numeric results. Solid line: fitting function, Eq. 2.28.

For other approximate soliton solutions with and without self-interactions, see Refs. [94, 95].

\footnotetext{
${ }^{1}$ When we derive $\alpha$ for each soliton solution by fitting Eq. 2.26 to the numerical results, we fix the core radius using Eq. 2.30 which is not smooth at $g^{2} \rho_{0}=1.5$. So the values of $\alpha$ with respect to $g^{2} \rho_{0}$ we obtain (circles) has a small fluctuation around that density.
} 


\begin{tabular}{lcc}
\hline & $g<0$ & $g>0$ \\
\hline$\alpha_{a}$ & 1.81823 & 3.96849 \\
$\alpha_{b}$ & 1.73925 & 2.11238 \\
$\alpha_{c}$ & 0.22478 & 0.143883 \\
\hline$\beta_{a}$ & 3.48601 & \\
$\beta_{b}$ & 1.29355 & $\beta=8$ \\
$\beta_{c}$ & 0.122718 & \\
\hline
\end{tabular}

Table 2.1: Best-fit parameters for the soliton density with attractive $(g<0)$ and repulsive $(g>0)$ self-interactions.

\subsection{Dynamical numerical simulations of ultralight bosons}

Obtaining analytical solutions of Eq.2.11 always is very difficult. Thus dynamical numerical simulations provide a useful way to solve Eq. 2.11. In fact, in addition to ultralight bosons models, numerical simulations help us to know the formation and evolution of galaxies for any other dark matter models from now on. Two properties define the simulations of ultralight bosons: initial conditions and dynamics. Modified initial conditions or dynamics help us understand the evolution of different situations for cold dark matter models. A large number of algorithms have been used in cosmological simulations. Two examples are spectral method and finite difference [41, 99, 124, 154, 155, 157]. The adaptive mesh refinement is widely used as well since the higher resolutions required.

Current research typically focus on large scales, $\sim \lambda$. However, the size of boson stars in halos, $\sim 1 /(m v)$, is much small than the $\lambda$, which therefore requires simulations to have very high resolution. This is a significant challenge for solving Eq. 2.11. For example, for the ultralight boson with mass, $m \sim 10^{-22} \mathrm{eV}$ and a velocity, $v \sim 100 \mathrm{~km} / \mathrm{s}$. The de Broglie wavelength is roughly equal to $1 \mathrm{kpc}$, which is much smaller than the large scale structure we tend to focus on, $\lambda>1 \mathrm{Mpc}$. For this reason, much of the research so far has involved simulations being limited to very small box sizes. Another method is to numerical solve the fluid formulation, Eq. 4.2. The variables of Eq. 4.2 are $\mathbf{v}$ and $\rho$ [123, 182]. Here, only the large scale flows need to be captured. This makes high spatial resolution unnecessary.

\subsection{Relationship of halos and non-compact boson stars}

We have known that both Dark matter halos and boson stars could be important forms of cold dark matter in the Universe. In 2014, Hsi-Yu Schive et al. studied 
the mass relationship between boson stars, $M_{c}$, and the mass of the surrounding haloes, $M_{h}$, in their cosmological simulations of the bosonic matter.

The dimensionless SP equation [188] with cosmological background can be written as

$$
\begin{aligned}
i \frac{\partial}{\partial \widetilde{\tau}} \widetilde{\psi} & =-\frac{1}{2} \nabla^{2} \widetilde{\psi}+a \widetilde{V} \widetilde{\psi}, \\
\nabla^{2} \widetilde{V} & =4 \pi m\left(|\widetilde{\psi}|^{2}-\widetilde{n}\right),
\end{aligned}
$$

where the time is normalized to $d \tau=\left(\frac{3}{8 \pi} H_{0}^{2} \Omega_{m 0}\right)^{1 / 2} a^{-2} d t$, and $\psi$ is normalized to $\left(\rho_{m 0} / m_{\psi}\right)^{1 / 2}$, the comoving length is normalized such that

$$
x_{\mathrm{comv}}=\left(\frac{3}{8 \pi} H_{0}^{2} \Omega_{m 0}\right)^{-1 / 4} m_{\psi}^{-1 / 2} .
$$

Here $\Omega_{m 0}$ is matter density parameter, $H_{0}$ is Hubble parameter, $\rho_{m 0}$ is background mass density, and $V$ the gravitational potential.

Through numerical simulations, Hsi-Yu Schive et al. found that all collapsed haloes form a soliton at their center. The solitions are the ground state solution of the SP equations, see Fig. 2.7][155]. The halo contains a central soliton of radius $r_{c} \sim \lambda_{\mathrm{dB}}$ and an outer profile coinciding with cold dark matter haloes, i.e. NFW profile. The task of halo modelling is to find transition radius $r_{\alpha}$, where the soliton profile (Eq. 2.25) transits to an NFW profile (Eq. 1.2). Hsi-Yu Schive et al. find the following relationship between solitons and their surrounding halos,

$$
M_{*}=a^{-1 / 3} M_{\text {halo' }}^{1 / 3}
$$

where they define $M_{*}$ to be the mass bound within a sphere of radius $r_{\text {core }}$ where the density drops to half of the central density, see Fig. 2.8

However, the transition from the solitons to the halos don't occurs at exactly $r_{\text {core, }}$ as shown in Fig 2.9. In fact, halos in simulations show the radii of solitons to be several times larger than $r_{\text {core, }}$ see Fig. 2.8 and 2.9. In 2018, Victor H. Robles et al. found that the halo of ultra-light bosons transitions from soliton to NFW at $r_{\alpha}=\alpha r_{\text {core. }}$. They found the value of $\alpha$ to be dependent on the mass of the halo with values of 2 and 3 for halos of masses $10^{10} M_{\odot}$ and $10^{12} M_{\odot}$ respectively [147].

In order to obtain the value of $\alpha$, we define the total density profile,

$$
\rho(r)= \begin{cases}\rho_{\mathrm{sol}}(r) & 0 \leq r \leq r_{\alpha} \\ \rho_{\mathrm{NFW}}(r) & r_{\alpha}<r \leq r_{\mathrm{vir}}\end{cases}
$$



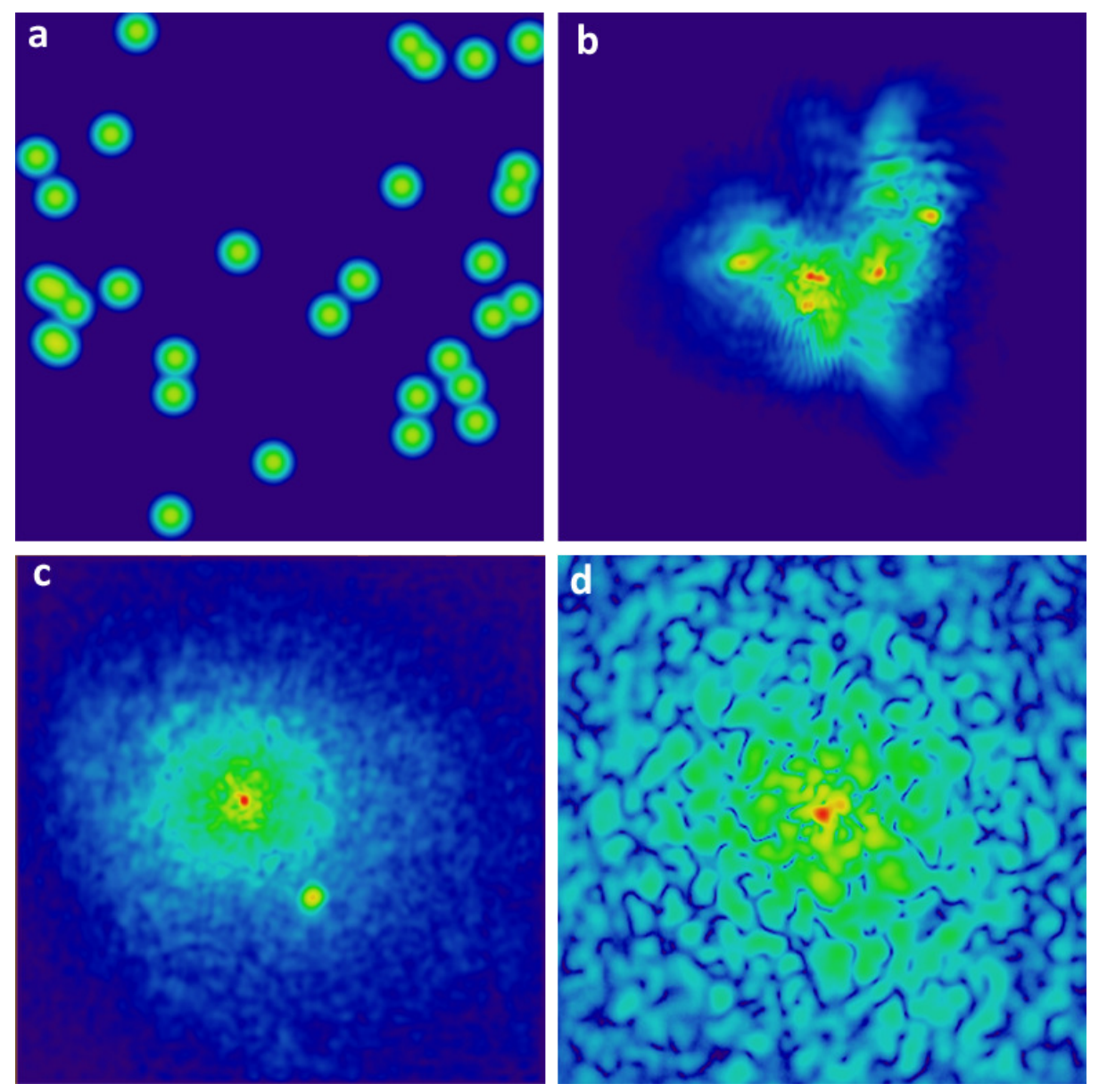

Figure 2.7: Snapshots of boson stars collision simulation. Panel (a) shows the density projection at the initial stages. Panels (b),(c) show the density projection at the intermediate stages. Panel (d) shows a dense core stay at the center of the halo. Reproduced from [155].

We impose continuity of density at $r_{\alpha}$

$$
\rho_{\mathrm{sol}}\left(r_{\alpha}\right)=\rho_{\mathrm{NFW}}\left(r_{\alpha}\right),
$$

and mass of halo within $r_{\text {vir }}$

$$
\begin{aligned}
M_{\text {halo }} & =4 \pi \int_{0}^{r_{\mathrm{vir}}} \rho(r) r^{2} d r \\
& =4 \pi \int_{0}^{r_{\alpha}} \rho_{\mathrm{sol}}(r) r^{2} d r+\int_{r_{\alpha}}^{r_{\mathrm{vir}}} \rho_{\mathrm{NFW}}(r) r^{2} d r .
\end{aligned}
$$

Cosmological simulations suggest values of $\alpha \sim 3$, see Fig 2.10. In addition, simulations illustrate that the transition occurs when the total quantum kinetic energy density is roughly equal to the potential energy density. 

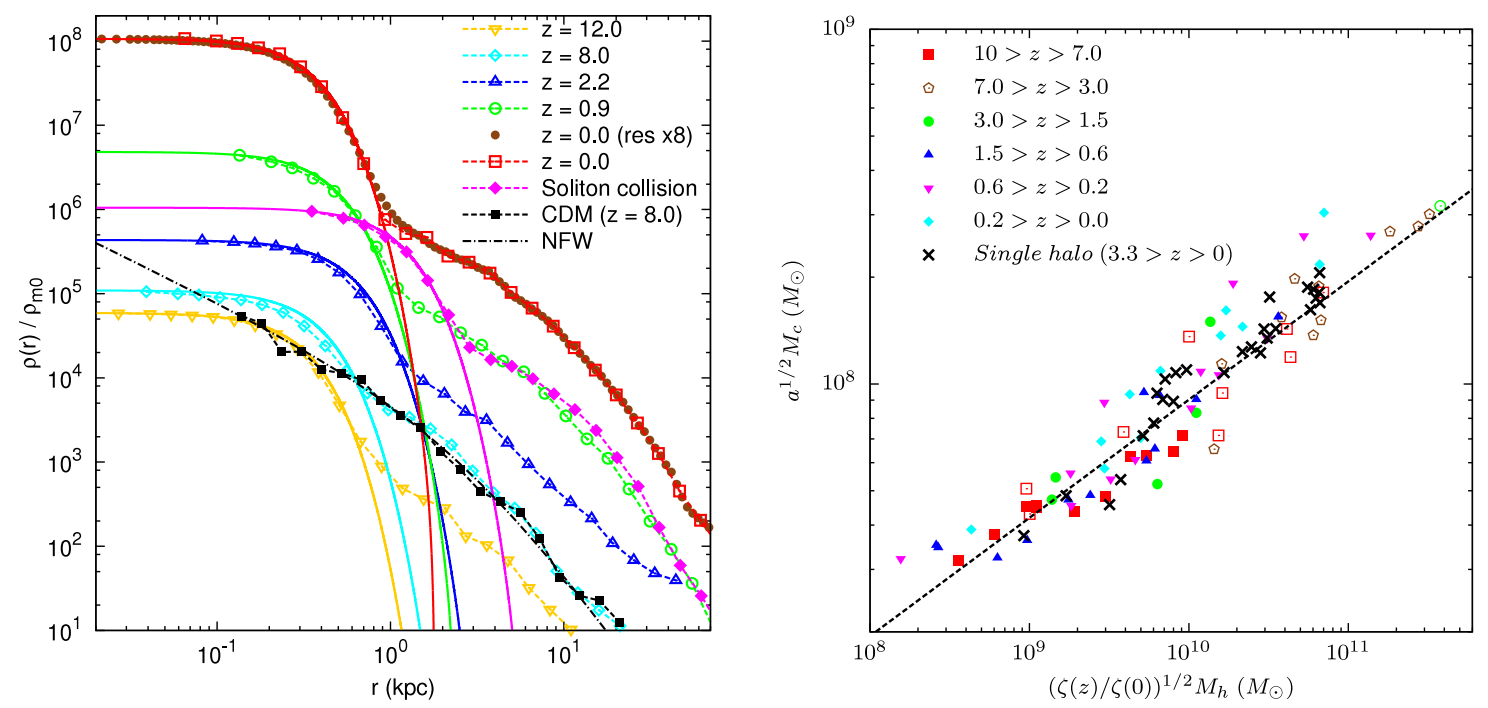

Figure 2.8: The left panel shows the density profile of halos. Dashed lines are the examples at different redshifts at $0<z<12$. The solid lines are soliton solution, see Eq 2.25. Dot-dashed line is the NFW profile given by Eq. 1.2 the right panel shows core-halo mass relation. Reproduced from Ref. [155]
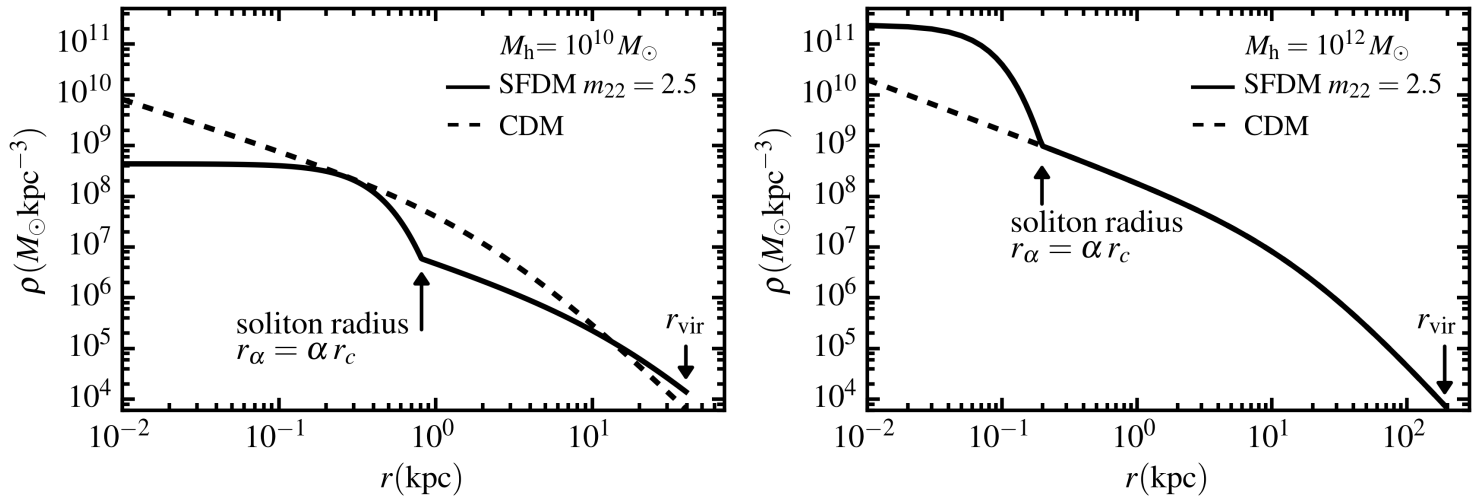

Figure 2.9: Solid line is the radial density profile of halo with ultra-light bosons. Dashed line is the profile of halo with no ultra-light bosons, i.e. not quantum effect. The left panel is a dwarf-size halo, $M_{\text {halo }}=10^{10} M_{\odot}$, and the right panel is a Milky-Way size halo, $M_{\text {halo }}=10^{12} M_{\odot}$. Reproduced from Ref. [147] 


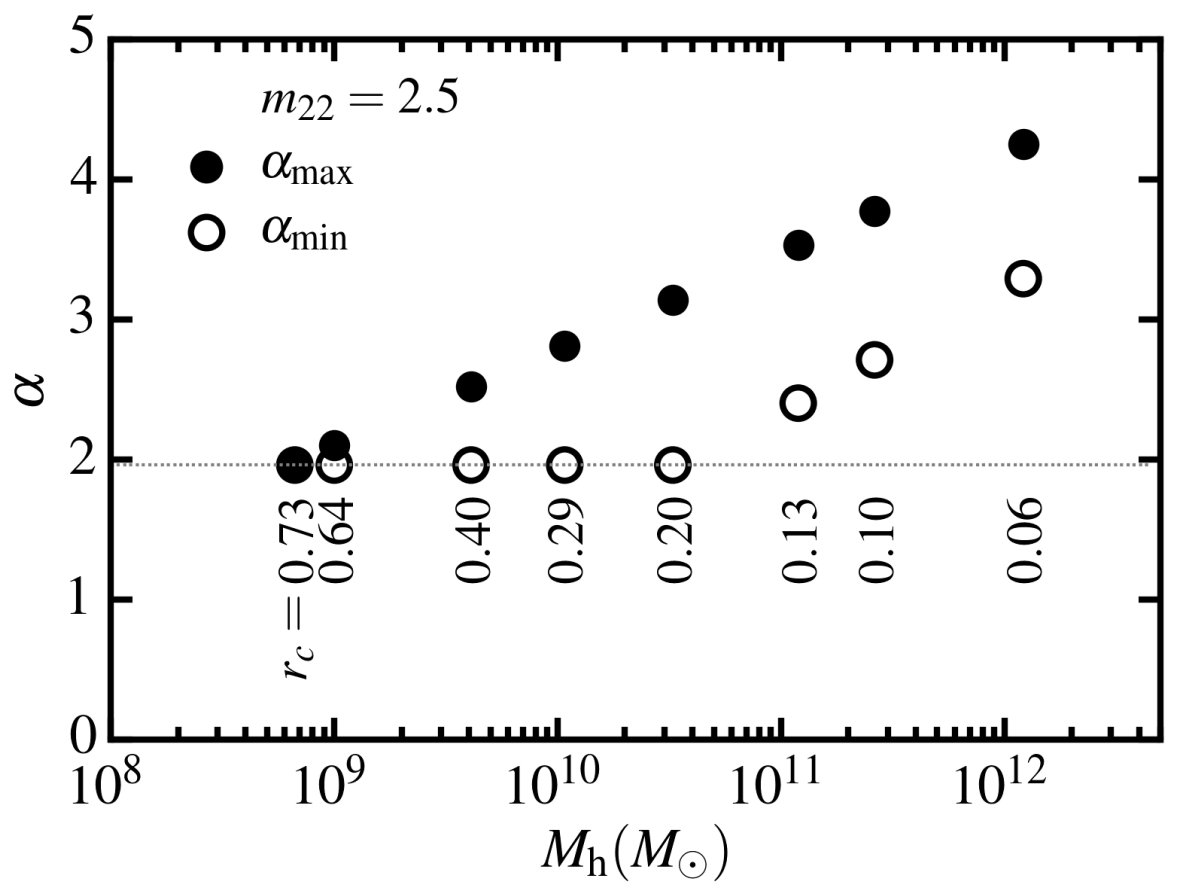

Figure 2.10: Range of $\alpha$. Reproduced from Ref. [147].

\subsection{Pseudo-Spectral Method}

The Pseudo-Spectral method is a good method to numerically solve time-dependent SP, GP or GPP equations since it converges faster and is more accurate that other numerical methods [65].

Pseudo-Spectral methods were first proposed by Kreiss and Oliger in 1972, which are numerical methods closely related to the spectral methods. Spectral methods are used to solve partial differential equations in applied mathematics and scientific computing, in which the solution is represent by a combination of orthogonal basis functions. In comparison, the pseudo-spectral method represent functions on a quadrature grid. The evaluation of certain operators are simplified. The speed of calculation can increase greatly as well.

The process of using Pseudo-spectral methods to solve Schrödinger equation is as follows. We write the kinetic operator as $K=-\frac{1}{2} \nabla^{2}$, potential operator as $W=V(x, t)$, and Hamiltonian as $H=K+W$. The evolution of $\psi$ is described by

$$
i \frac{\partial \psi(\vec{x}, t)}{\partial t}=H \psi(\vec{x}, t) .
$$

This equation can be solved iteratively

$$
\psi(\vec{x}, t+d t)=e^{-i H d t} \psi(\vec{x}, t),
$$


where $d t$ is time step. Due to the canonical commutation relation of $K$ and $W$, we have the algorithms of the general form

$$
e^{-i(K+W) d t}=\prod_{j}^{N}\left(e^{-i k_{j} d t K} e^{-i v_{j} d t V}\right)
$$

where $k_{j}$ and $v_{j}$ are parameters. This equation has the general Campbell-BakerHausdorff expansion,

$$
\begin{aligned}
& \prod_{i}^{N}\left(e^{t_{i} d t K} e^{v_{i} d t V}\right)=\exp \left(d t \left(e_{K K}+e_{V} V+d t e_{K V}[K, V]+d t^{2} e_{K K V}[K,[K, V]]\right.\right. \\
& \left.\left.+d t^{2} e_{V K V}[V,[K, V]]+\ldots . .\right)\right)
\end{aligned}
$$

where all the error coefficients $e_{T}, e_{T V}, e_{V T V}$, etc, are calculable functions of $k_{i}, v_{i}$ where $e_{T}=\sum_{i=1}^{N} k_{i}, e_{V}=\sum_{i=1}^{N} v_{i}$.

\subsubsection{Second Order Algorithm}

For the second order algorithm, we can take a specific level of expansion

$$
\begin{aligned}
& e^{-i(K+W) d t} \approx \exp \left(-i \frac{1}{2} K d t\right) \exp (-i W d t) \exp \left(-i \frac{1}{2} K d t\right) \\
& =\exp \left(d t\left(e_{T} K+e_{V} W-\frac{1}{24} d t^{2}[K,[K, W]]+\frac{1}{12} d t^{2}[W,[W, K]] \ldots . .\right)\right)
\end{aligned}
$$

This relationship can be proven easily when we Taylor expand left term of this equation,

$$
e^{-i H d t}=e^{-i(K+W) d t}=1-i(K+W) d t-\frac{1}{2}\left(K^{2}+K W+W K+W^{2}\right) d t^{2}+\widetilde{O}\left(d t^{3}\right) .
$$

Combing the equations

$$
\begin{gathered}
e^{-i K d t}=1-i K d t-\frac{1}{2} K^{2} d t^{2}+\widetilde{O}\left(d t^{3}\right) \\
e^{-i \frac{1}{2} W d t}=1-i \frac{1}{2} W d t-\frac{1}{8} W^{2} d t^{2}+\widetilde{O}\left(d t^{3}\right),
\end{gathered}
$$

we obtain

$$
\begin{aligned}
& \exp \left(-i \frac{1}{2} K d t\right) \exp (-i W d t) \exp \left(-i \frac{1}{2} K d t\right) \\
& =1-i(K+W) d t-\frac{1}{2}\left(K^{2}+K W+W K+W^{2}\right) d t^{2}+\widetilde{O}\left(d t^{3}\right)
\end{aligned}
$$

Thus we can confirm that this equation is accurate to second order on $d t$. We can calculate $K$ in Fourier space, and $W$ in real space. 


\subsubsection{Fourth Order Algorithm}

Compared with the second order algorithm, fourth order algorithm shows better accuracy. Similarly, we expand $e^{-i(K+W) d t}$ in forth order

$$
\Gamma_{M 1}=\ldots \exp \left(d t t_{0} W\right) \exp \left(d t v_{1} K\right) \exp \left(d t t_{1} W\right) \exp \left(d t v_{2} K\right) \exp \left(d t t_{2} W\right)
$$

where $t_{0}, v_{1}, t_{1}, v_{2}, t_{2}$ are parameters, and "..." means that it is symmetric with respect to the right hand term of the equation. The order condition requires that [42]

$$
\begin{aligned}
w & =\sqrt{3-12 v_{1}+9 v_{1}^{2}} \\
t_{1} & =\frac{1}{2}-t_{2} \\
t_{2} & =\frac{1}{4}\left(1-\sqrt{\frac{9 v_{1}-4+2 w}{3 v_{1}}}\right), \\
v_{0} & =1-2\left(v_{1}+v_{2}\right), \\
v_{1} & =\frac{121}{3924}(12-\sqrt{471}) \\
v_{2} & =\frac{1}{6}-4 v_{1} t_{1}^{2} .
\end{aligned}
$$

As before, we calculate $K$ in Fourier space, and calculate the $W$ in real space.

\subsubsection{Fast Fourier transform}

$K=-\frac{1}{2} \nabla^{2}=\frac{1}{2} p^{2}$ needs to be calculated in Fourier space, but $W=V(x, t)$ is computed in real space. Thus we use the Fourier transform $\psi(x)$ to $\psi(p)$, and inverse Fourier transform $\psi(p)$ to $\psi(x)$. Fast Fourier transform (FFT) provide a good way to do these processes efficiently.

FFT was first derived in 1805, but was not widely used until 1965. FFT is a method of fast calculating the discrete Fourier transform of a sequence or similarly inverse discrete Fourier Transform (IFFT). In addition to computational cosmology, FFT is widely used in music, engineering as well as many other fields. One of the most popular fast Fourier transform algorithms is the Cooley-Tukey algorithm. We can regroup the items in the DFT formula on the time (spatial) domain, which 
is decimation-in-time,

$$
\begin{aligned}
& X[p]=\sum_{n=0}^{N-1} x[n] e^{-j(2 \pi n p / N)}, \quad p=0,1, \ldots, N-1 \\
& =\sum_{n=\operatorname{even}} x[n] W_{N}^{n p}+\sum_{n=\text { odd }} x[n] W_{N}^{n p} \\
& =\sum_{n=0}^{N / 2-1} x[2 r] W_{N}^{2 r p}+\sum_{n=0}^{N / 2-1} x[2 r+1] W_{N}^{(2 r+1) p} \\
& =\sum_{n=0}^{N / 2-1} x[2 r] W_{N}^{2 r p}+W_{N}^{p} \sum_{n=0}^{N / 2-1} x[2 r+1] W_{N}^{(2 r) p} \\
& =\sum_{n=0}^{N / 2-1} x[r] W_{N / 2}^{2 r p}+W_{N}^{p} \sum_{n=0}^{N / 2-1} x[2 r+1] W_{N / 2^{\prime}}^{(r) p}
\end{aligned}
$$

where we set $W_{N}^{n p}=e^{-j(2 \pi n p / N)}$. This method can reduce the amount of computation cost from $O\left(n^{2}\right)$ to $\mathcal{O}(n \log n)$, where $n$ is the data size. Of course, This is just an example of the Fastest Fourier Transform, we still can regroup the items in the DFT formula in the frequency domain. There are many other algorithms such as Prime-factor FFT algorithm. Each of these have their own advantages and can be applied to different situations. Therefore, it is very important to choose a suitable algorithms for our purposes. We use the Fastest Fourier Transform in the West (FFTW) which supports a variety of algorithms and choose the best one [67]. FFTW is a software library developed by Matteo Frigo and Steven G. Johnson.

\subsection{Application of codes}

Based on previous mentioned numerical methods, we have developed a code to simulate the bosonic system with self-gravity and possible self-interactions. The code is highly stable thus very suitable for studying the long-time evolution of the system. We have also added the support for GPU acceleration and optimize the GPU memory cost. This provides a $6-7 \times$ speed-up compared to the pure CPU code.

\subsubsection{Quantum harmonic oscillator}

Firstly, we use a single quantum harmonic oscillator in one dimension to test the accuracy of our codes in solving the dimensionless Schrödinger equation,

$$
i \frac{\partial}{\partial \widetilde{t}} \widetilde{\psi}=-\frac{1}{2} \frac{\partial}{\partial \widetilde{x}} \widetilde{\psi}+\frac{1}{2} \widetilde{\omega}^{2} \widetilde{x}^{2}
$$


where $\frac{1}{2} \widetilde{\omega}^{2} \widetilde{x}^{2}$ is the potential energy of the quantum harmonic oscillator and $\widetilde{\omega}$ is its angular frequency. We choose one of the bound solution as our initial condition,

$$
\widetilde{\psi}(x, 0)=\left(\frac{1}{\pi}\right)^{\frac{1}{4}} \exp \left(-\frac{\widetilde{x}^{2}}{2}\right) .
$$

Numerically solving Eq. 2.55, we find the simulation results $|\widetilde{\psi}(\widetilde{x}, \widetilde{t} \sim 100)|$ coincide with the initial condition $|\widetilde{\psi}(\widetilde{x}, \widetilde{t} \sim 0)|$, as shown in Fig 2.11. Thus we can confirm our codes is reliable since the $|\psi(x, t)|=\psi(x, 0)$ for this bound solution.

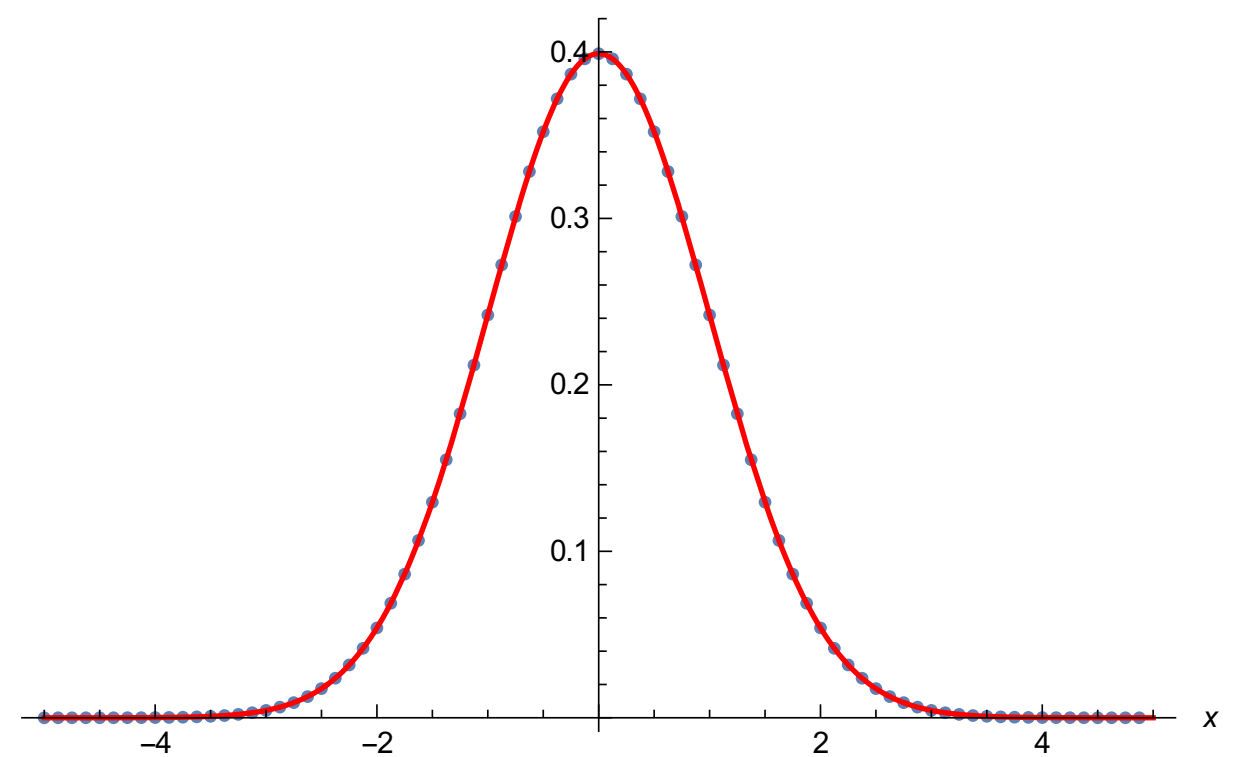

Figure 2.11: Comparison of the initial condition $|\widetilde{\psi}(\widetilde{x}, \widetilde{t} \sim 0)|$ (black dotted line) and simulation results $|\widetilde{\psi}(\widetilde{x}, \widetilde{t} \sim 100)|$ (red solid line)

\subsubsection{Colliding of two boson stars}

The process of the collision of two equal mass boson stars with certain velocities has been studied by Argelia Bernal et al in 2006 [25]. They found that as two boson stars move toward each other, they form interference pattern where they overlap. After a while, if the two boson stars are not bound, they will pass through each other completely and continue to travel apart independently. This is a very credible conclusion. It was also confirmed in several subsequent studies [92, 157]. This therefore provides us with a very good way to verify the code.

Argelia Bernal et al. consider two boson stars with gravity. Thus we consider Schrödinger-Poisson equation in our simulation. The dimensionless equation of 
SP equations are

$$
\begin{gathered}
\left(-\frac{1}{2} \widetilde{\nabla}^{2}+\widetilde{V}(\widetilde{\vec{x}}, \widetilde{t})\right) \widetilde{\psi}(\widetilde{\vec{x}}, \widetilde{t})=i \frac{\partial}{\partial \widetilde{t}} \widetilde{\psi}(\widetilde{\vec{x}}, \widetilde{t}), \\
4 \pi \delta \widetilde{\rho}=\nabla^{2} \widetilde{V}
\end{gathered}
$$

We use the same parameters as Bernal et al. in Ref [25]. Setting the distance between two solitons in $\widetilde{x}$ axis to 30 , and the speed to 3.0 in $\widetilde{x}$ axis respectively, we have

$$
\widetilde{\psi}(\widetilde{\vec{x}}, \widetilde{t})=\sqrt{\widetilde{\rho}} e^{i \widetilde{S}}
$$

The wavefunction of left boson star is given by

$$
\psi(\overrightarrow{\vec{x}}, 0)=\frac{1}{\left(1+\left(0.230\left(\sqrt{(\widetilde{x}+15)^{2}+\widetilde{y}^{2}+\widetilde{z}^{2}}+15\right)\right)^{2}\right)^{4}} e^{3 i \widetilde{x}} .
$$

The wavefunction of right boson star is given by

$$
\psi(\overrightarrow{\widetilde{x}}, 0)=\frac{1}{\left(1+\left(0.230\left(\sqrt{(\widetilde{x}-15)^{2}+\widetilde{y}^{2}+\widetilde{z}^{2}}-15\right)\right)^{2}\right)^{4}} e^{-3 i \widetilde{x}}
$$

Here the velocity, $\widetilde{v}=\nabla \widetilde{S} / m$.

Fig. 2.12 shows the process of collision of the two boson stars. We can see two boson stars start with an initial separation of of 30 at $\widetilde{t}=0$. Then they gradually move towards each other until they eventually collide. At time $=5$, the two boson stars overlap completely and produce a clear interference pattern. Subsequently, they pass through and move away from each other. We also compare the interference pattern with theoretical prediction, and find them to be in close agreement, as shown in Fig. 2.13, All of these simulation results match the results of Argelia Bernal et al. [25]

\subsubsection{Code accuracy}

In the previous section, we introduced second-order and fourth-order algorithms. We can use the relative error of the total energy conservation during the collision of two boson stars to compare their accuracy. In all runs, the energy is computed as

$$
\begin{aligned}
E[\psi] & =\int\left[\frac{1}{2 m^{2}}|\nabla \psi|^{2}+\frac{1}{2} V|\psi|^{2}\right] \mathrm{d}^{3} x \\
& =\int \frac{1}{2 m^{2}}(\nabla \sqrt{\rho})^{2} \mathrm{~d}^{3} x+\int \frac{\rho}{2} v^{2} \mathrm{~d}^{3} x+\int \frac{\rho}{2} V \mathrm{~d}^{3} x+\int \frac{\rho}{2} g|\psi|^{2} \mathrm{~d}^{3} x \\
& =K_{\rho}+K_{v}+W_{\text {gravity }}+W_{\text {self }},
\end{aligned}
$$



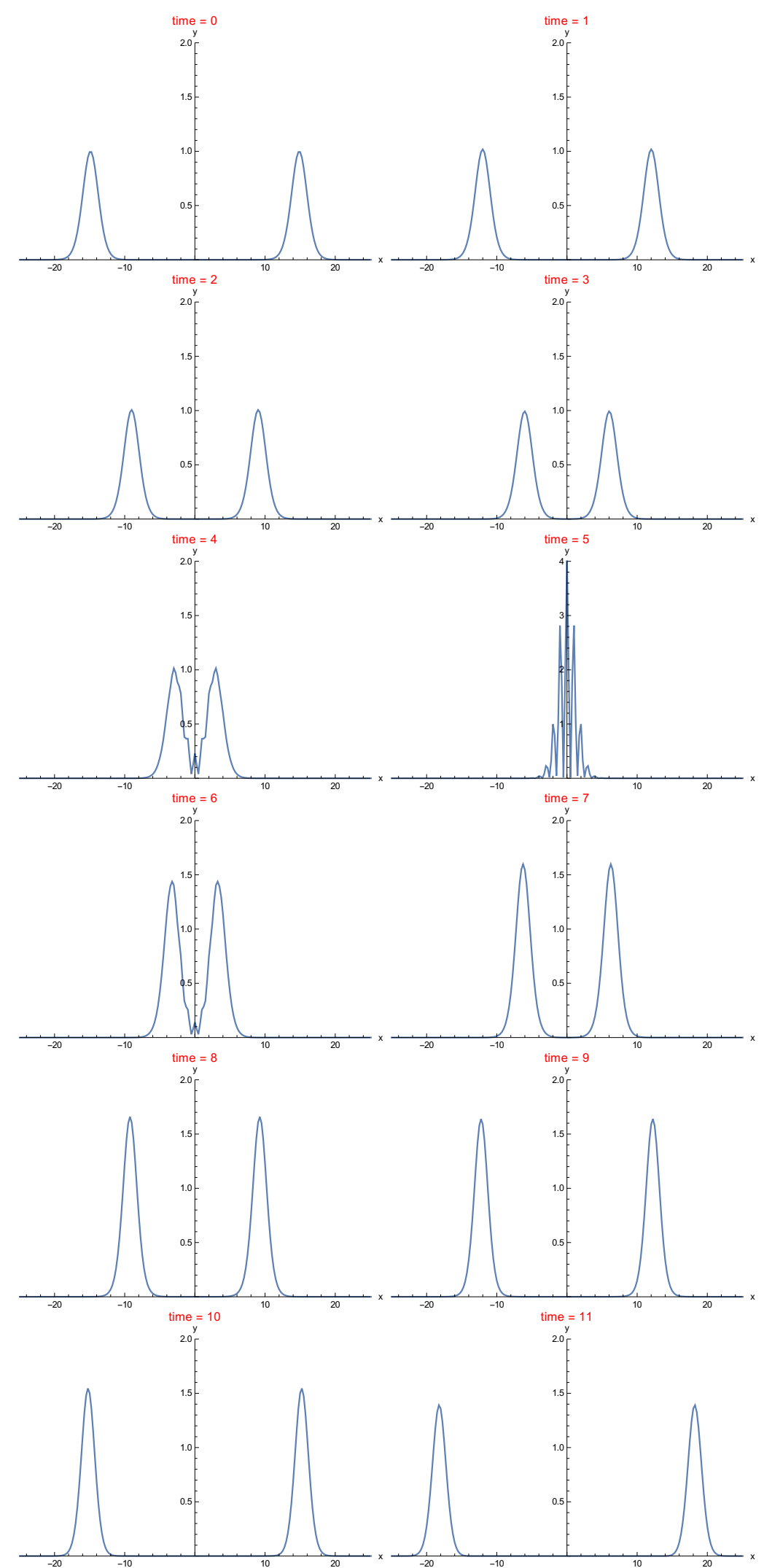

Figure 2.12: Collision of two boson stars. This shows the density evolution $|\widetilde{\psi}(\widetilde{x}, 0,0, \widetilde{t})|^{2}$. 


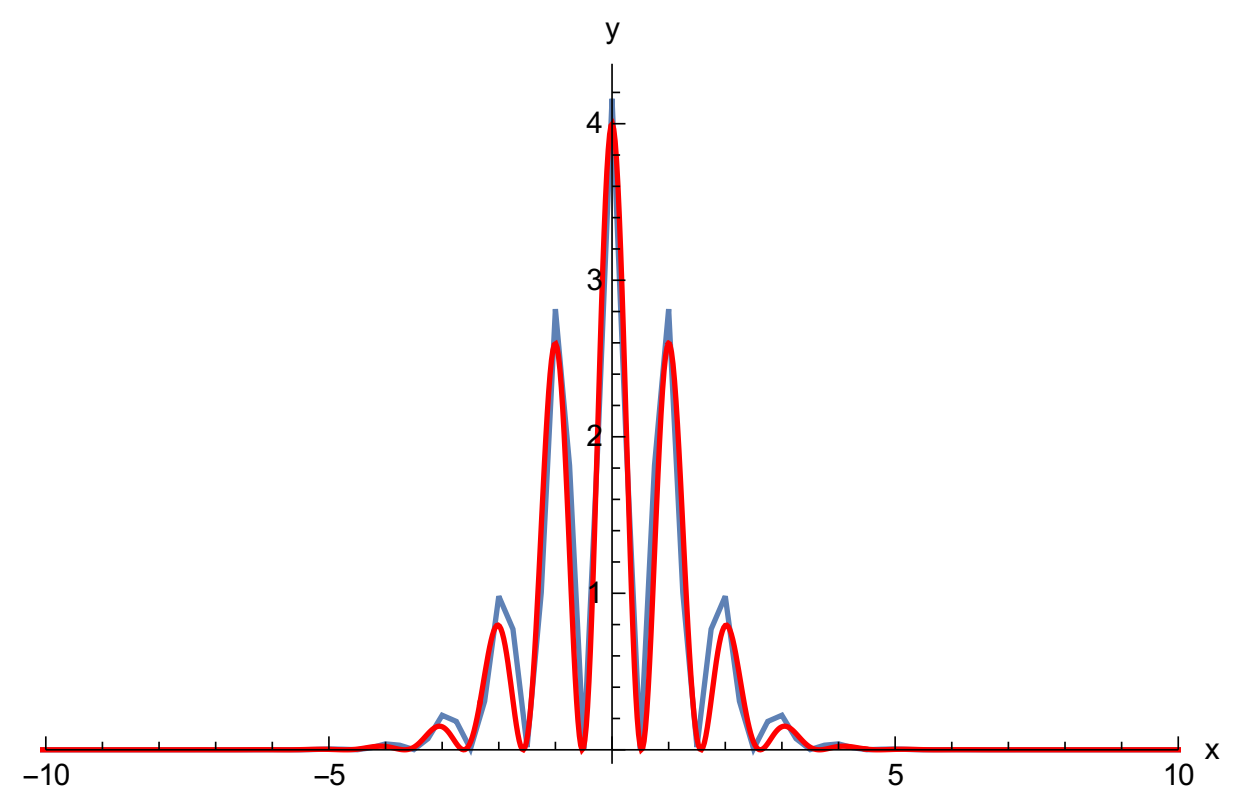

Figure 2.13: Comparison of the theoretical and simulation results of $|\widetilde{\psi}(\widetilde{x}, 0,0, \widetilde{t})|^{2}$ at $\widetilde{t} \sim 5$. The red line is the theoretical results and blue line is simulation results.

where $K_{\rho}$ is the quantum pressure, $K_{v}$ is the 'classical' kinetic energy, $W_{\text {gravity }}$ the gravity energy, and $W_{\text {self }}$ is the internal energy. The internal energy is zero in this test since we consider the case with no self-interactions $(g=0)$.

Fig. 2.14 shows the relative error of the total energy conservation with respect to time for the second order algorithm and the fourth order algorithm. We can see that the fourth order algorithm is more accurate compared with the second order algorithm despite the fact that the time step size is $\pi$ times larger.

\subsection{Conclusion}

As hypothetical astronomical objects, the study of boson stars is a good way to understand the nature of ultra-light bosons as a candidates of dark matter. For compact boson stars, we have the possibility to detect their unique feature from observations. One example is the possibility of the existence of a boson star in Sgr A* which fits the dynamical data and observed luminosity of the center of the Galaxy [177, 183]. In addition, current electromagnetic telescopes can not detect dark matter. However, gravitational wave astronomy may provide us a method for detecting dark matter since the gravitational signature from merger of compact boson stars would be distinct from other astrophysical invisible objects such as black holes [27]. 


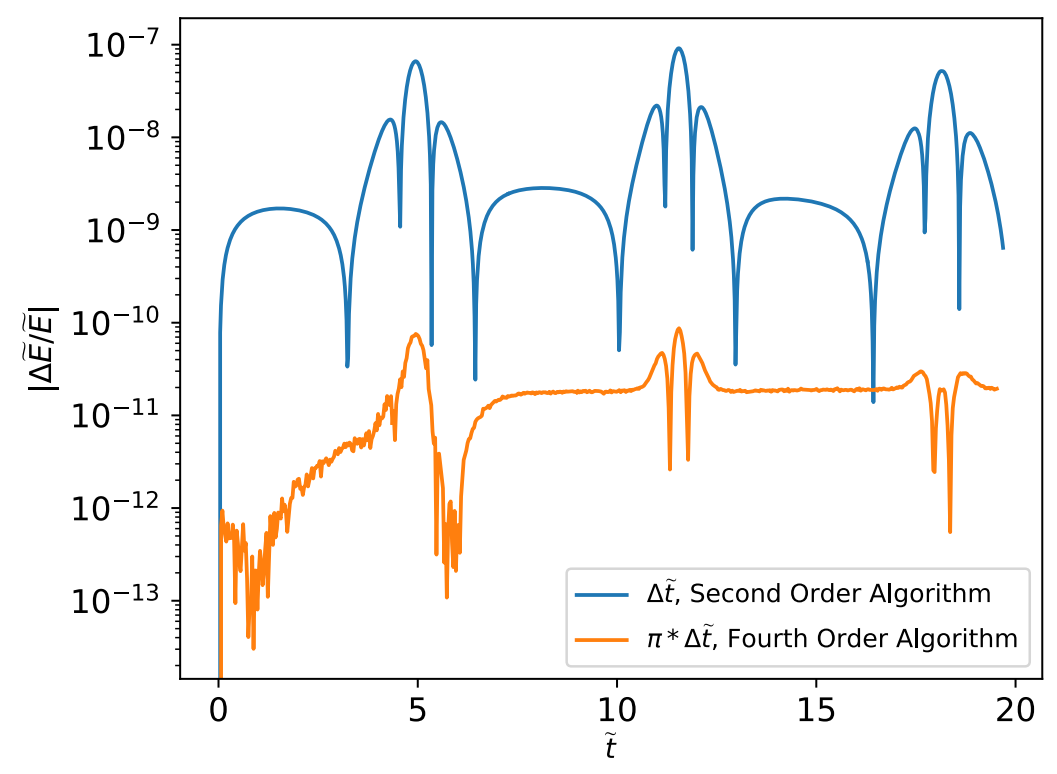

Figure 2.14: Relative errors of the total energy with respect to time for different order algorithm.

The study of non-compact boson stars is currently a very popular and active area of research. Here, we try to obtain numerical solutions of boson stars with different self-interaction by solving time-independent GPP equations and derive their best-fit functions. These results have many applications. We have shown the relationship between the masses of dark matter halos and their central boson stars to be $M_{*} \propto \mathrm{M}_{\text {halo }}^{1 / 3}$. Additionally, we have shown that the transition radius, where the density profile of the halo transits from a soliton profile to a NFW-like profile, $r_{\alpha} \sim 3 r_{\text {core. }}$

In order to solve the time-dependent GPP equations, we introduce the pseudospectral method. In fact, as partial differential equations, the analytical solutions of the various time-dependent GPP equations, are always very difficult to obtained. In order to solve this problem, several numerical methods have been proposed, such as finite-difference and pseudo-spectral methods. However, the latter has been shown to provide both higher accuracy and stability [65, 141]. This is one reason why it is widely used in dynamical simulation of ultralight bosons in cosmology [25, 60, 71, 99]. Here, we implement this method with high-order time integration. Using GPU acceleration and optimizing the GPU memory cost, we confirm that the code produces more accurate results using less computing time compared 
to previous second-order time integration algorithm.

Firstly, we solve the simplest model, evolving the bound solution of quantum harmonic oscillator in one dimension. We find that the evolution of bound solution in numerical simulations almost perfectly fit the theoretical predictions, $|\psi(x, t)|=$ $\psi(x, 0)$.

Secondly, we test the classical simulations in the study of boson stars, colliding of two boson stars with certain velocity, which was first studied in 2006 [25]. By comparing to existing results, including theoretical prediction and simulation results, we find that in all of our simulations the numerical code we developed works as expected. As shown in Fig. 2.12, the two solitons travel towards each other, producing a distinctive interference pattern, before passing through each other completely and traveling apart in opposite directions. This interference pattern also fits the theoretical prediction very well. Furthermore, we also find our code produces more accurate results by studying the energy conservation.

These tests provide us confidence to repeat and verify more research with higher accuracy and efficiency. Of course, we have more chances to obtain reliable and interesting new research results in the future. 


\section{Chapter 3}

\section{Formation and evolution of boson stars and halos/miniclusters}

\subsection{Theory of condensation of bosons}

In 2018, Levkov et al. found that the gravitational interactions between distant bosons are important for the condensation of bosons [99]. A Boltzmann collision process isn't suitable to describe the evolution of a statistical ensemble of bosons with gravity when they are too dense. The reason is that we cannot treat the collision as a process only involving two bosons for this situation.

Based on this theory, Kay Kirkpatrick et al. argue that for bosons with the short range self-interaction, we still can not use the Boltzmann collision process to describe it as well [93]. This is because the de Broglie wavelength is not small than cube-root of the bosons number density $n^{1 / 3}$ despite the fact that the mean free path of self-interactions small compared to $n^{1 / 3}$. Thus we have ratio of length scales,

$$
\lambda_{\mathrm{dB}} n^{1 / 3} \gg 1 \text {. }
$$

To deal with this situation, we describe the systems by the Wigner function, $f_{W}(\mathbf{x}, \mathbf{p})$,

$$
f_{W}(\mathbf{x}, \mathbf{p})=\int d^{3} \xi \exp (-i \mathbf{p} \xi)\left\langle\psi(\mathbf{x}+\xi / 2) \psi^{*}(\mathbf{x}-\xi / 2)\right\rangle .
$$

Taking the time derivative of Eq. 3.2 and using Eq. 2.11, we obtains dynamical equation for $f_{W}(x)$,

$$
\begin{aligned}
\frac{D f}{D t}= & \partial_{t} f_{W}+\frac{\mathbf{p}}{m} \nabla_{\mathbf{x}} f_{W} \\
= & 2 \operatorname{Im} \int d^{3} \xi e^{-i \mathbf{p} \xi}\left\langle\psi(\mathbf{x}+\xi / 2) \psi^{*}(\mathbf{x}-\xi / 2)\right\rangle \\
& {[V(\mathbf{x}+\xi / 2)-V(\mathbf{x}-\xi / 2)] }
\end{aligned}
$$


Note that the gravitational potential is given by

$$
V \mathbf{x}=4 \pi G m \int d^{3} \mathbf{x}^{\prime} \Delta_{\mathbf{x}-\mathbf{x}^{\prime}}^{-1}\left(\left|\psi_{\mathbf{x}^{\prime}}\right|^{2}-n\right),
$$

where $\Delta^{-1}$ is the Green's function of the poisson equation in Eq.2.11. This can be expanded by Wick's theorem since we will set initial distribution $\psi$ as a Gaussian random field, see Sec 3.2.1. The non-Gaussianities occur later due to weak gravity or self-interactions. Thus the Wick theorem is still approximately true,

$$
\left\langle\psi_{\mathbf{x}_{1}} \psi_{\mathbf{x}_{2}}^{*} \psi_{\mathbf{x}_{3}} \psi_{\mathbf{x}_{4}}^{*}\right\rangle=\left\langle\psi_{\mathbf{x}_{1}} \psi_{\mathbf{x}_{2}}^{*}\right\rangle\left\langle\psi_{\mathbf{x}_{3}} \psi_{\mathbf{x}_{4}}^{*}\right\rangle+\left\langle\psi_{\mathbf{x}_{1}} \psi_{\mathbf{x}_{4}}^{*}\right\rangle\left\langle\psi_{\mathbf{x}_{3}} \psi_{\mathbf{x}_{2}}^{*}\right\rangle+\left\langle\psi_{\mathbf{x}_{1}} \psi_{\mathbf{x}_{2}}^{*} \psi_{\mathbf{x}_{3}} \psi_{\mathbf{x}_{4}}^{*}\right\rangle_{\text {conn }}
$$

where the connected part $\langle\cdot\rangle_{\text {conn }} \sim O(G)$ is small and gives $O\left(G^{2}\right)$ contribution to Eq. 3.3

We substitute Eq. 3.3 and 3.4 into Eq. 3.3 and express all two-point functions via $f_{W}$, Eq. 3.2. This gives non-local equation,

$$
\begin{aligned}
\frac{D f}{D t}= & \partial_{t} f_{W}+\frac{\mathbf{p}}{m} \nabla_{\mathbf{x}} f_{W} \\
= & \operatorname{St} f_{w}+2 m \operatorname{Im} \bar{V}\left(x+\frac{i}{2} \nabla_{\mathbf{p}}\right) f_{W}+\frac{2 \pi G m^{2}}{(2 \pi)^{6}} \operatorname{Im} \int d^{3} \xi \xi^{\prime} \mathbf{p} \mathbf{p}^{\prime} \\
& \Delta^{-1} \xi^{i \xi^{\prime} q-i \xi^{\prime} q^{\prime}} \times f_{W}(\mathbf{p}+\mathbf{q})(x+\xi / 2) f_{W}\left(\mathbf{p}+\mathbf{q}^{\prime}\right)\left(x+\xi^{\prime} / 2\right),
\end{aligned}
$$

where $\bar{V}=\langle V\rangle$ is the mean potential, which satisfies

$$
\Delta \bar{V}=4 \pi G m\left(\int \frac{d^{3} \mathbf{p}}{(2 \pi)^{3}} f_{W}(\mathbf{x})-n\right) .
$$

We note that Eq. 3.5 are T-symmetry if $\operatorname{St} f_{w}=0$. Therefore, the two-point functions are time-reversal symmetric, which make them unable to dominate the relaxation of bosons in the system. Thus only the scattering integral St $f_{W} \sim O\left(G^{2}\right)$ contributes to the relaxation of bosons. Eq. 3.5 takes the form of

$$
\partial_{t} f_{W}=\operatorname{St} f_{W}
$$

Thus, the scattering integral determines the condensation time of bosons in the system.

For a halo with radius, $R$, we can Taylor expand $f_{w}$ in $\Delta x / R \ll 1$ and $\Delta p \lambda_{d B} \ll$ 1 if $\delta x \ll R$. Finally, the term St $f_{W}$ can be find as

$$
\partial_{t} f_{W}+\nabla_{p} \mathcal{H} \nabla_{x} f_{W}-\nabla_{x} \mathcal{H} \nabla_{p} f_{W}+O\left(\epsilon^{2}\right)=\operatorname{St} f_{W},
$$


where

$$
\mathcal{H}=\frac{p^{2}}{2 a^{2} m}+m\langle V\rangle
$$

is the one-particle Hamiltonian with the averaged gravitational potential $\langle V\rangle$, the coherence length, $\lambda_{d B}=1 / v_{\text {vir }}$ with $v_{\text {vir }}$ the virial velocity. For $\langle V\rangle$, we have $\nabla^{2}\langle V\rangle=4 \pi G m\left(\int d^{3} p f_{W}-n\right)$. In addition, $O\left(\epsilon^{3}\right)$ terms are time-reversal symmetric as well. This means that $\mathrm{St} f_{W}$ is the only term which can cause the condensation of bosons. The remaining terms, apart from $O\left(\epsilon^{2}\right)$, in Eq. 3.7. are the same as the Vlasov-Poisson (VP) equations. The gravitational scattering time scale dominates the scattering integral,

$$
\text { St } f_{W} \simeq f_{W} / \sigma,
$$

where $f_{W} \approx \frac{6 \pi^{2} n}{m v^{3}}$ and $\sigma$ is the transport Rutherford cross section of gravity or self-interaction scattering.

\subsubsection{Transport Rutherford cross section}

Calculating the transport Rutherford cross section of gravitational interaction is not a difficult task. Using the Born-Oppenheimer approximation, we can obtain the differential cross section

$$
\frac{d \sigma_{\text {gravity }}}{d \Omega}=-\frac{2 m^{2}}{q^{2}}\left|\int_{0}^{L} r V_{\text {gravity }}(r) \sin (q r) d r\right|^{2},
$$

where $q=4 k^{2} \sin ^{2} \frac{\theta}{2}, m$ is the mass of the particles, $k=m v$ is the particles momentum, $V_{\text {gravity }}(r)=-G m / r$ is the gravitational potential, $\sigma_{\text {gravity }}$ is transport Rutherford cross section, $\Omega$ is the solid angle [30]. Finally, we can obtain $\sigma_{\text {gravity }}=$ $8 \pi(m G)^{2} \log (m v R) / v^{4}$. The condensation time of bosons is given by

$$
\tau_{\text {gravity }}=\frac{4 b \sqrt{2}}{\sigma_{\text {gravity }} v n f}=\frac{b \sqrt{2}}{12 \pi^{3}} \frac{m v^{6}}{G^{2} n^{2} \log (m v R)^{\prime}},
$$

which is given in terms of the radius of miniclusters, $R$, characteristic velocity, $v$, and density, $n$, of the minicluster, $f=6 \pi^{2} n /(m v)^{3}$ is the phase-space density, and $b$ is a fitting parameter. The value of $b$ is related to the types of initial mass and momentum distribution [99.155].

However, there is currently some disagreements regarding the transport Rutherford cross section for self-interaction. Levkov et al. believe that $\sigma_{\text {self }}=m^{2} g^{2} /(2 \pi)$ [99]. However, Kay Kirkpatrick et al. claim that $\sigma_{\text {self }}$ is proportional to $g$ [92]. To date, 
no one has verified their results through theory or numerical simulations. This dispute is not the focus of our research topic. The details of this disagreement are therefore beyond the scope of this thesis.

If we assume that $\sigma_{\text {self }}$ is proportional to $g^{2}$, the ratio of condensation time of self-interaction to gravity can be written as

$$
\frac{\tau_{\text {gravity }}}{\tau_{\text {self }}} \sim \frac{\sigma_{\text {self }}}{\sigma_{\text {gravity }}} \sim \frac{g^{2} v^{4}}{16 \pi^{2} G^{2} \log (m v L)},
$$

where $\tau_{\text {self }}$ is the condensation time due to self-interaction. Using Eq. 3.12, we can estimate the effect of self-interaction and gravity on the condensation of boson stars.

For example, for a system of typical QCD axions with velocity, $v \approx 10^{-9}$, and decay constant $f_{a} \approx 10^{11} \mathrm{GeV}$ [164], we have $\tau_{\text {gravity }} / \tau_{\text {self }} \ll 1$, thus gravity plays a much more important role in the condensation process.

On the other hand, if the claim by Kay Kirkpatrick et al. [92] is correct, i.e. the relaxation rate due to self-interaction is proportional to $|g|$ rather than $g^{2}$, a much shorter condensation time for self-interaction is expected. However, for typical QCD axions gravity still dominates the condensation process.

\subsection{Simulation of condensation of Bosons}

\subsubsection{Initial conditions}

We set up two isotropic initial conditions in the momentum space:

- Gaussian-distributed bosons $[99],\left|\psi_{\vec{p}}\right|^{2}=\widetilde{N} e^{-\tilde{p}^{2}}$ in a periodic box of size $L$, with random phases $\arg \tilde{\psi}_{\tilde{p}}$, where $N \equiv n L^{3}$ is number of non-relativistic bosons in the box. Performing an inverse Fourier-transforming $\psi_{\vec{p}}$, we obtain an isotropic and homogeneous initial distribution $\psi(\vec{x}, 0)$.

- Delta-distribution bosons [99], $\left|\psi_{\vec{p}}\right|^{2}=N \delta\left(|\vec{p}|-m v_{0}\right)$ in a periodic box of size $L$. Performing an inverse Fourier transform on $\psi_{\vec{p}} e^{i S}$ with $S$ a random phase, we also obtain an isotropic initial distribution in position space, $\psi(\vec{x}, 0)$.

\subsubsection{Formation of miniclusters and condensation of bosons stars with only gravity}

For the bosons with only gravity $(\widetilde{g}=0)$, the GPP equation can be simplified to SP equations. Using Gaussian-distributed bosons as initial conditions, we nu- 
merically evolve the SP equation using Pseudo-spectral method. The formation of Boson stars is shown in Fig. 3.1. We can see a dense object occur at $\widetilde{t}=1.1 * 10^{6}$, see the right panel of Fig. 3.1. The left panel of Fig. 3.2 shows the radial density profile of this object from this most dense point. It can be seen that the profile coincides with the density profile described in Eq. 2.25. Thus we confirm that a boson star has been condensed within the box. The evolution of maximum $\widetilde{\psi}$ is shown in the right panel of Fig. 3.2. We see that its value remains approximately constant until $\widetilde{t}=1.1 * 10^{6}$. Furthermore, after nucleation, boson stars start to acquire more mass from the surrounding field, with an initial mass growth rate $\propto t^{1 / 2}$.
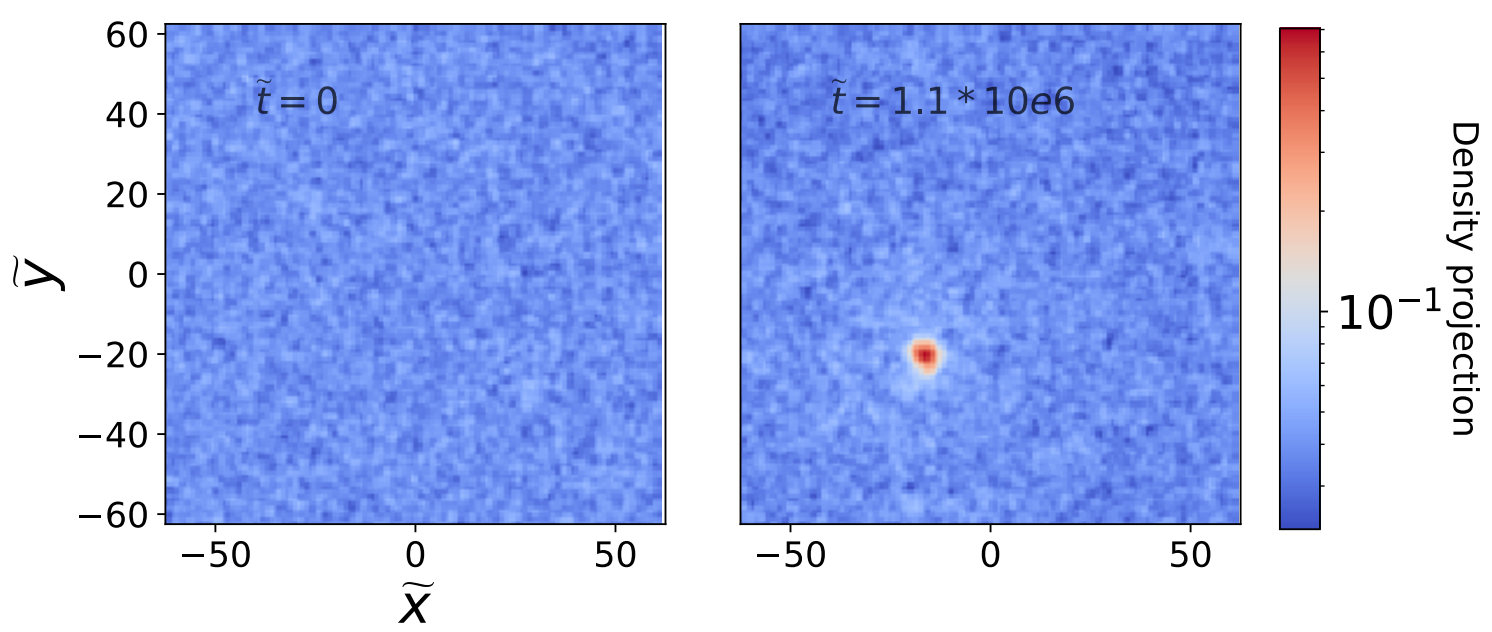

Figure 3.1: Formation of Bose star from random field with initial distribution from $\psi(\vec{x}, 0) \propto e^{-\widetilde{p}^{2}}$. Total mass $\widetilde{N}=628.3$, box size $\widetilde{L}=125$. Left: Projection of density at $\widetilde{t}=0$. Right: Projection of density at $\widetilde{t}=1.1 * 10^{6}$.

So far we have assumed that a homogeneous ensemble in the box correctly describes central parts of dark matter halos. Now, we study the isolated halos/miniclusters themselves to verify this assumption. In order to study isolated halos/miniclusters, we run simulations in a box of size $\widetilde{L}>2 \pi / \widetilde{k}_{J}$ where $\widetilde{k}_{J}=(4 \widetilde{n})^{1 / 4}$ is the dimensionless Jeans wavenumber, since non-relativistic boson gas forms clumps at scales larger than $2 \pi / \widetilde{k}_{J}$ due to Jeans instability [90]. This initial distribution follows from the uncertainty principle: exact knowledge of $\vec{p}$ gives complete uncertainty in $\vec{x}$. Numerically solving the SP equations, we observe the formation of of a boson star and its surrounding halo/minicluster. One example is shown in Fig. 3.3 with a box size $\widetilde{L}=18$ and total mass $\widetilde{N}=1005.3$. We can see a minicluster forming gradually from $\widetilde{t} \approx 10$ to $\widetilde{t} \approx 30$. After that, a dense and nearly spherically symmetric object 

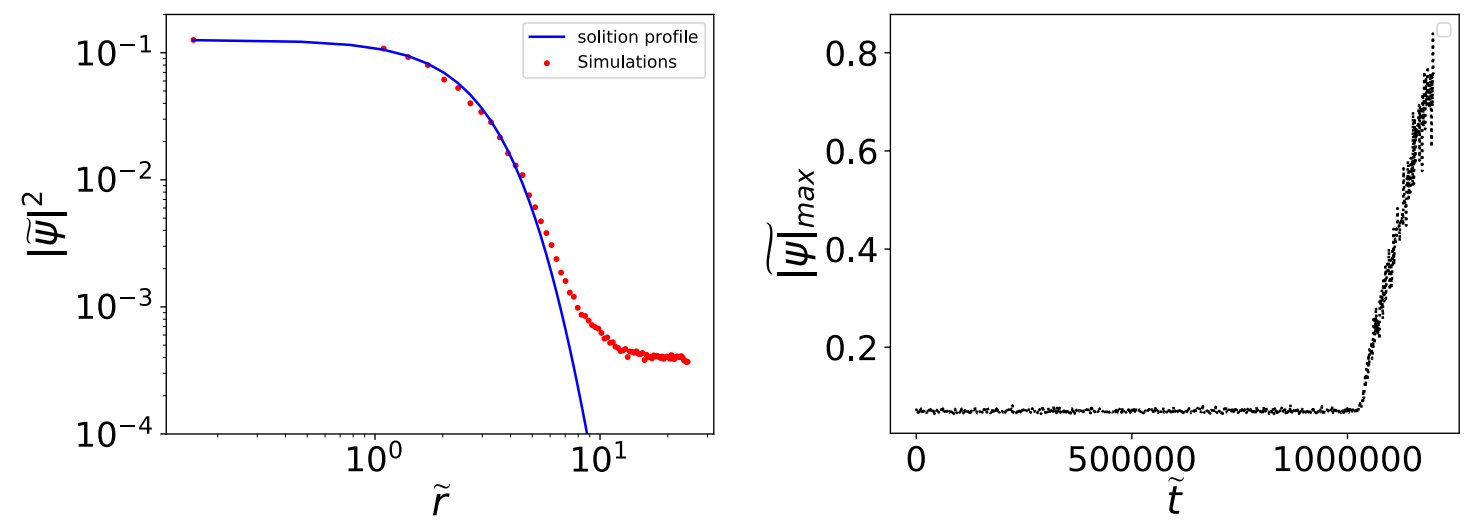

Figure 3.2: Formation and growth of Bose star from random field with initial distribution from $\psi(\vec{x}, 0) \propto e^{-\widetilde{p}^{2}}$. Total mass $\widetilde{N}=628.3$, box size $\widetilde{L}=125$. Left: Radial profile of the object (colored dots) in Fig. 3.1 solitonic profiles (solid lines) as given by Eq. 2.25 with the same central densities at $\widetilde{t}=1.1 * 10^{6}$. Right: Maximum of $\widetilde{\psi}$ over the box as a function of time.

appears and grows in the center of the minicluster. We find that the radial density profile of the minicluster from this most dense point coincides with the density profile of a soliton solution at $0<\widetilde{r}<1$ (soliton density profiles are described in Eq. 2.25), and a power law at $\widetilde{r}>1$ (see Fig. 3.4). We also find that there is always one, and only one, boson star formed in each minicluster ${ }^{1}$. The region outside the boson star has a radial density profile consistent with cold dark matter on scales larger than the de Broglie wavelength, and with granular structure below it. These results are fully consistent with results of Refs. [60, 99, 155].

By repeating such simulations many times with unique randomly generated initial conditions, we also verify the condensation time is fit to Eq.3.11, see Fig. 3.5 (a,b), and $b=0.7$ for Delta-distribution bosons, $b=0.9$ for Gaussian-distributed bosons.

\subsubsection{Saturation of boson stars in axion miniclusters}

After condensation, boson stars have been shown to acquire mass from the surrounding gas of particles, with the subsequent growth rate

$$
M_{*}(t) \simeq M_{*, 0}\left(\frac{t-\tau_{\text {gravity }}}{\tau_{\text {gravity }}}\right)^{1 / 2},
$$

where $M_{*}$ is the mass of the boson star, $M_{*, 0}$ is the mass of boson star at $t=$ $\tau_{\text {gravity }}[99]$.

\footnotetext{
${ }^{1}$ We will see later that there are situations in which mutliple boson stars are formed.
} 

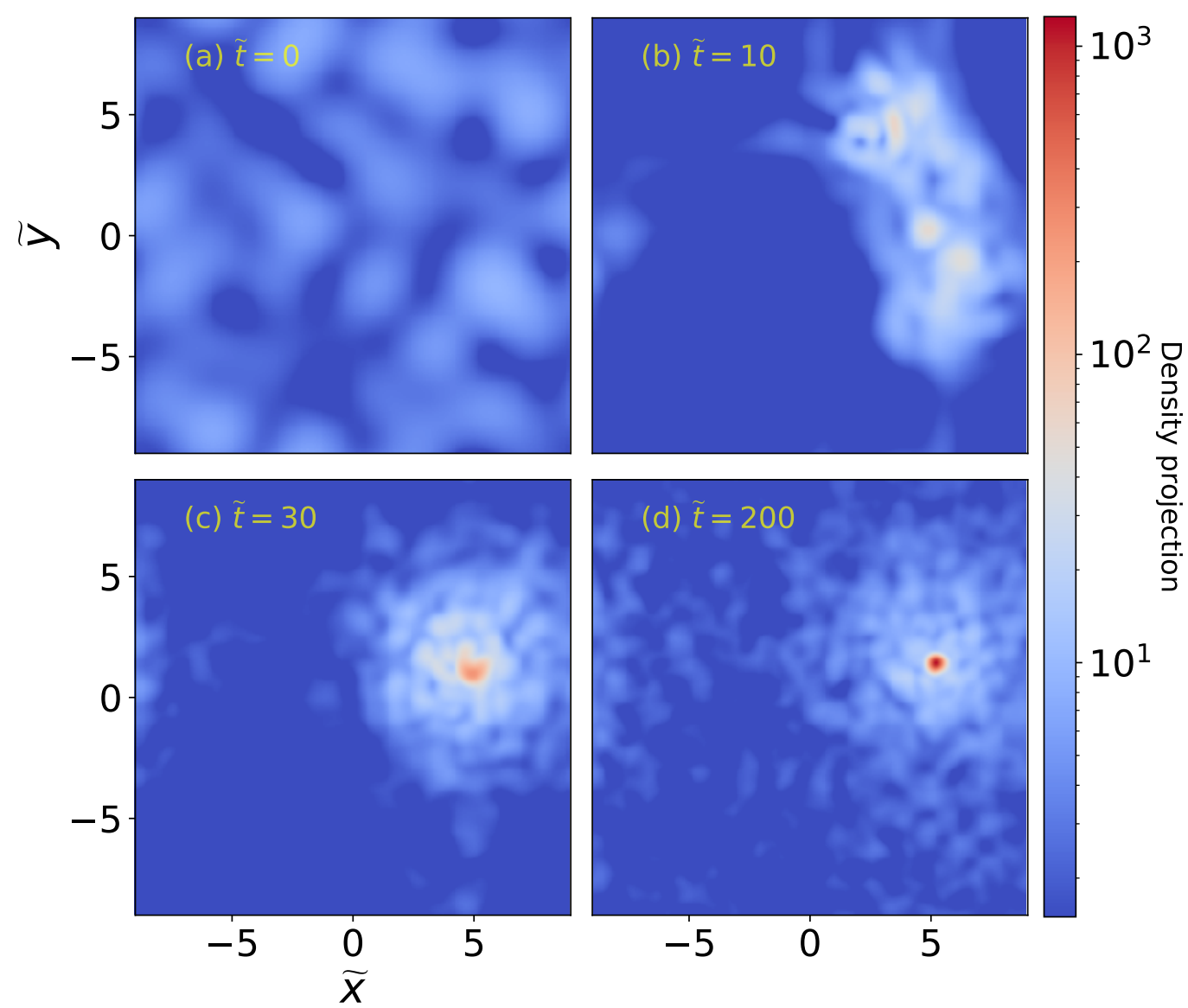

Figure 3.3: Snapshots of the density field from one simulation with $\widetilde{N}=1005.3$, $\widetilde{L}=18$. (a) Projected density at the initial time. (b) Projected density at $\widetilde{t}=10$, which shows that minicluster is forming in the box. (c) Projected density at $\widetilde{t}=30$. (d) Projected density at $\widetilde{t}=200$. A single dense object is visible at the centre of the minicluster. 


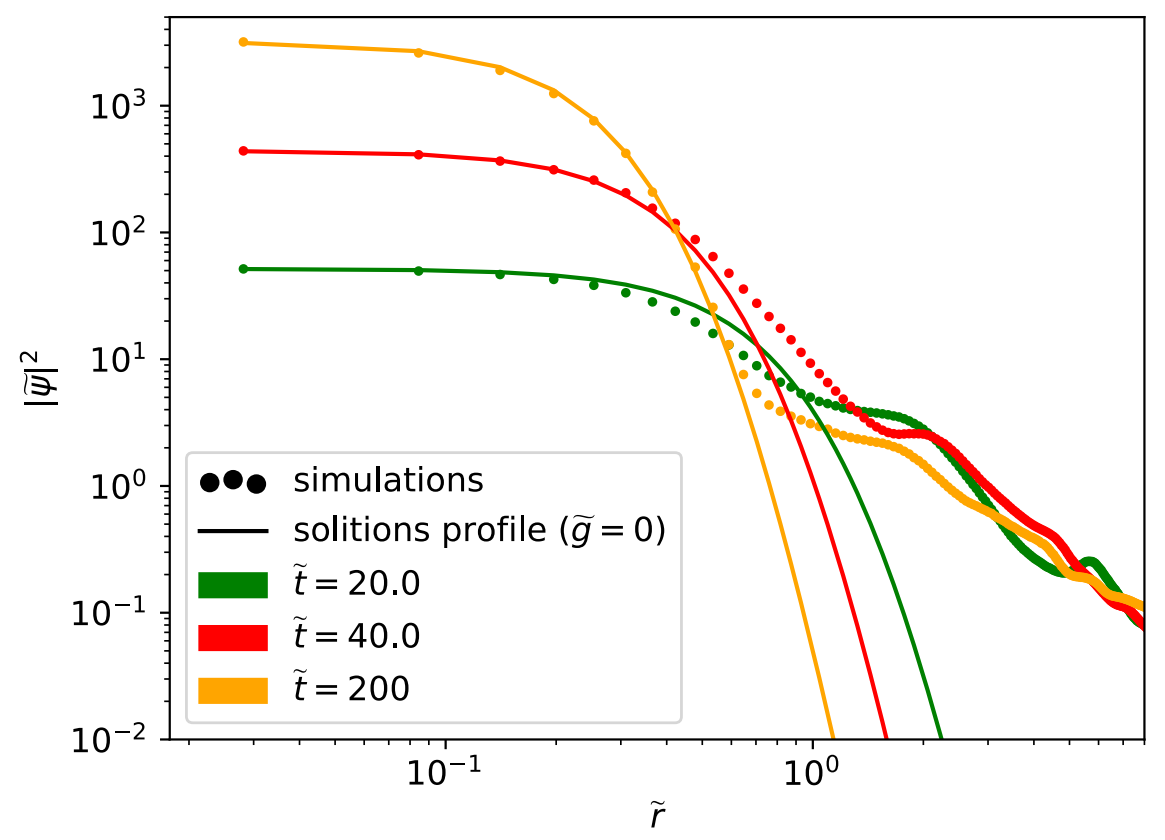

Figure 3.4: Density profiles of the minicluster at different times (colored dots) compared with solitonic profiles (solid lines) as given by Eq. 2.25 with the same central densities.

The question arises as to whether the growth in Eq. 3.13 continues forever or saturates. We know immediately after a boson star has been formed, its growth rate is in accordance with Eq. 3.13. As this boson star grows, surrounding bosons become gravitationally bound to it in a halo or atmosphere (the minicluster surrounding the star). The halo surrounding the boson star contains granular structure on the scale of the de Broglie wavelength, which can be modelled as consisting of transient "quasi particles" [81, 155]. As the boson star grows in mass, its radius

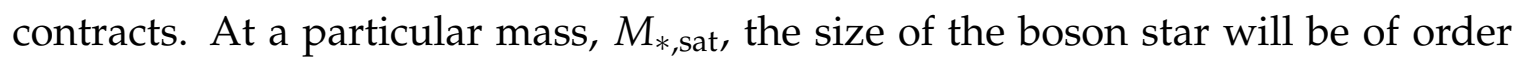
of the granular structure. At this time, it has been conjectured that the hot atmosphere will reach virial equilibrium with the star, causing the mass growth to slow down [60]. The transition has been predicted occur at $v_{\text {vir } *} \approx v_{\text {halo }}$ [60], where $v_{\text {vir* }}$ and $v_{\text {halo }}$ are the viral velocity of the boson star and minicluster respectively. We call this time the saturation time, $\tau_{\text {sat }}$. The saturation time is estimated by considering the viral velocity in the gravitational potential of the soliton, which is approximately given by. [81]

$$
v_{\text {vir* }}\left(M_{*}\right) \simeq G M_{*} m .
$$




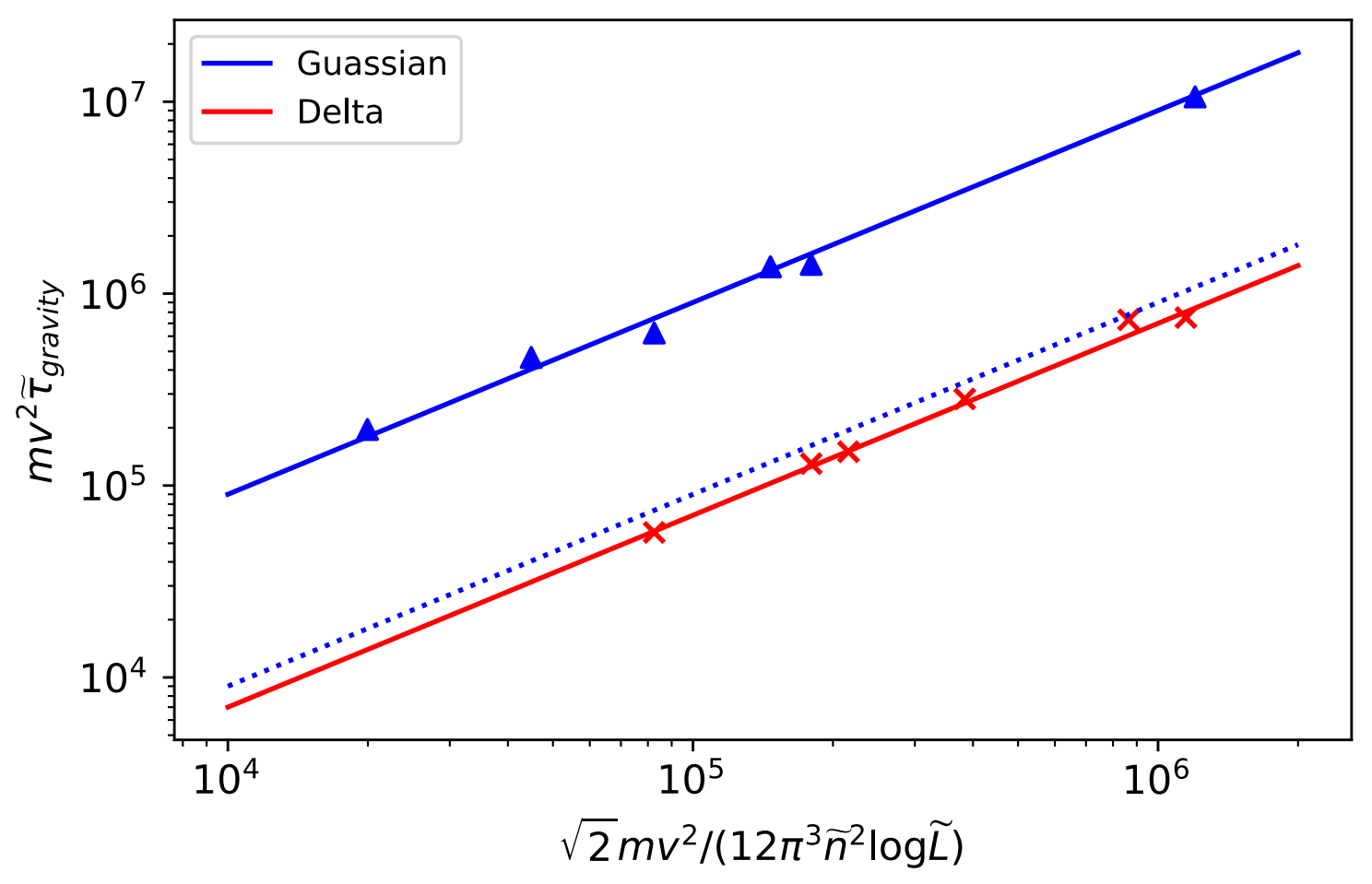

Figure 3.5: Time to Bose star formation in the cases of Gaussian and Delta initial distributions. Note that the $\tau_{g r}$ of Gaussian initial distributions are shifted upwards $\left(\tau_{g r} \rightarrow \tau_{g r} * 10\right)$ for clearly purposes. The dotted line is real $\tau_{g r}$ obtained by Eq. 3.11 
Exploiting the fact that $\left(M_{*, \text { sat }} / v_{\text {sat }, *}\right)^{3 / 4}=(m G)^{-3 / 4}$, and combining this with Eq. 3.13 gives

$$
M_{*}\left(t_{\text {star }}\right) \simeq M_{* \text {,sat }}\left(\frac{t_{\text {star }}}{\tau_{\text {sat }}}\right)^{1 / 8}
$$

with

$$
\tau_{\text {sat }}=\frac{b \sqrt{2}}{12 \pi^{3}} \frac{m v_{*, \text { sat }}^{6}}{G^{2} n^{2} \log \left(m v_{\text {halo }} R_{\text {halo }}\right)},
$$

where $M_{* \text {,sat }}$ is the boson star mass at the saturation time, $v_{* \text {,sat }}=v_{\text {vir } *}\left(M_{* \text {,sat }}\right)$, $R_{\text {halo }}$ is the radius of the halo and $v_{\text {halo }}$ is the virial velocity of the halo [60].

Due to computational limitations, the prediction of the saturation of boson stars has not been verified previouly [60,99]. In the rest of this subsection, by running a large number of numerical simulations past the estimated saturation time $t>\tau_{\text {sat }}$, we are able to demonstrate that the growth of boson stars in miniclusters indeed saturates as predicted.

Fig. 3.6 shows the evolution of mean, normalised and stacked mass of boson star for our ensemble of simulations. The boson stars form at $t \approx \tau_{\text {gravity }}$. We find

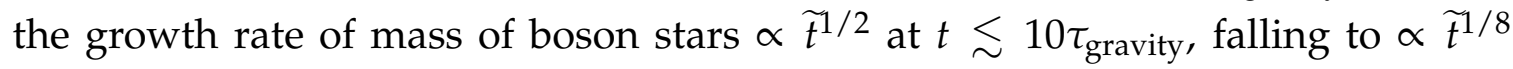
at the the saturation time. Therefore, we know when the size of the boson star becomes smaller than the granular structure in the surrounding halo, the boson star growth saturates and drops to $\propto \widetilde{t}^{1 / 8}$ at the transition time, as predicted by Eq. 3.15. Therefore, the saturation of boson star growth indeed occurs in our system, and the asymptotic mass growth rate of the boson star matches the theoretical prediction [60].

Furthermore, we find that during the final stages of evolution, the mass of boson star at saturation time is proportional to $\widetilde{N}^{\alpha}$, where $\widetilde{N}$ is the total mass in the simulation box. To find the best-fit parameter $\alpha$, we define the mean fractional deviation $\sigma_{\alpha}$ as

$$
\sigma_{\alpha}^{2}=\frac{1}{N_{\text {bin }}} \sum_{i}\left(\frac{\widetilde{M}_{*, i}(\alpha)-\widetilde{M}_{* \text { mean }}(\alpha)}{\widetilde{M}_{*, \text { mean }}(\alpha)}\right)^{2} .
$$

Here $\widetilde{M}_{*, i}(\alpha)$ is the boson star mass normalized by $\widetilde{N}^{\alpha}, \widetilde{M}_{*, \text { mean }}(\alpha)$ is the mean value within a specific time bin (see Fig. 3.6 for more explanations). We only include the data at $t / \tau_{\text {gravity }}>20$. Figure 3.7 shows $\sigma_{\alpha}$ calculated for each set of simulations with the same box size (colored lines) and the combined $\sigma_{\alpha}$ including all simulations (black line). We find that the mean fractional deviation is minimized at $\alpha \sim 0.45$, which is close to the value we would expect from the core mass-halo mass relation found in previous studies [155], i.e. $\tilde{M}_{*} \propto \widetilde{M}_{\text {halo }}^{1 / 3} \propto \widetilde{N}^{1 / 3}$. 


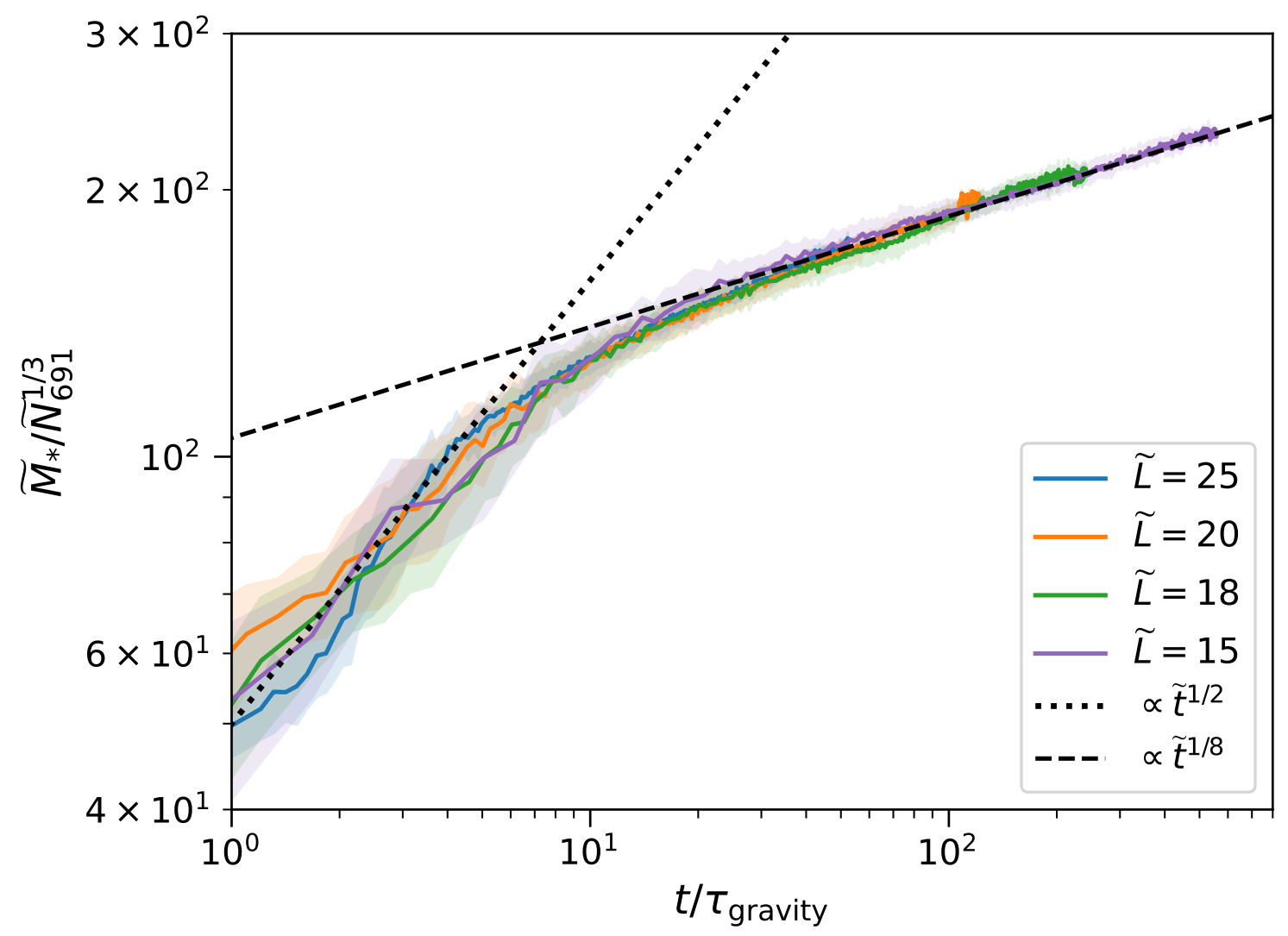

Figure 3.6: The mean stacked mass of boson stars evolution (solid lines) for different box sizes $\widetilde{L}=25,20,18,15$ and total mass $\widetilde{N}=691,754,817,880,942,1005,1131$. The data from simulation with the same box size $\widetilde{L}$ but different total mass $\widetilde{N}$ are divided into 500 time bins. The shaded regions show the $1-\sigma$ intervals. The time and mass of boson stars are normalized by the condensation time, $\tau_{\text {gravity }}$ and the total mass, $\widetilde{N}_{691}^{1 / 3}$, where $\widetilde{N}_{691}=\widetilde{N} / 691$. Note that here $\tau_{\text {gravity }}$ is computed using Eq. (3.11) for the initial configuration, i.e. $R=L, v=v_{0}$, and $n=N / L^{3}$, to avoid ambiguities in the definitions of halo radius and density. 


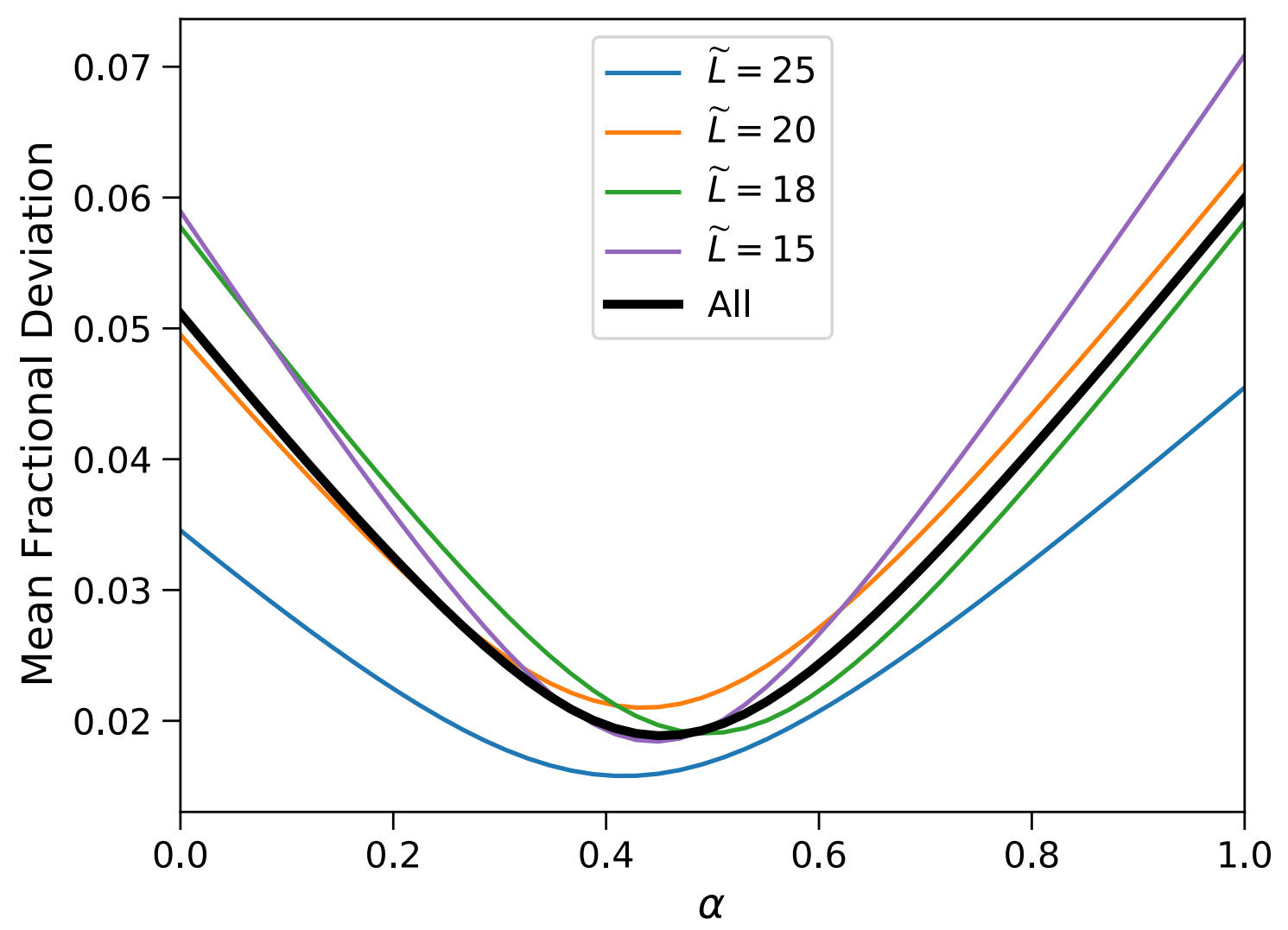

Figure 3.7: Mean fractional deviation from the fitting formula $\widetilde{M}_{*, \text { sat }} \propto \widetilde{N}^{\alpha}$. The colored lines show the results from simulations grouped by box size, while the black line shows the results combing all simulations. 


\subsection{Axions with self interaction}

\subsubsection{Axions with weak attractive self interaction}

Levkov et al. predict that sufficiently weak attractive self-interactions, like those of the QCD axion, have a negligible effect on boson star formation [99]. However, this prediction has not been directly demonstrated. For bosons with weak attractive self-interaction, such as QCD axions with $v \approx 10^{-9}$, and decay constant $f_{a} \approx 10^{11} \mathrm{GeV}$, where $f_{a}=-1 / \sqrt{-12 g}$, we obtain an estimate on the selfinteraction coupling of $\widetilde{g} \approx-10^{-2}$. We run some simulations at this range of $\widetilde{g}$. One of these simulations is shown in Fig. 3.8. We can see the process of formation of the minicluster and condensation of the boson star. This process is similar to the pure gravity case shown in Fig. 3.3. The radial density profiles of the minicluster and analytic profiles of soliton with and without self-interactions are given in Fig. 3.9 and ref Eq.2.26 and Eq.2.25, respectively. We discover that the radial density profile of the minicluster coincides with the density profile of a soliton solution at $0<\widetilde{r}<1$. We also see that the case with the correct value of $\widetilde{g}$ provides a better fit. The evolution of maximum density from simulations with different strength of self-interactions compared with the case without self-interactions is shown in Fig. 3.10. These results support the theoretical prediction of Ref. [99] that gravity dominates the system and the effect of self-interactions is negligible in the early stages of boson star evolution.

As the central density continues to grow, however, the effect of self-interactions becomes increasingly important. We thus find that at large values of $|\widetilde{g}|$, the boson stars collapse at a critical mass, see Fig. 3.10 [38-40, 101]. Above the critical mass, the boson star is unstable to perturbations. The attractive self-interaction in Eq. 2.17 overcomes the quantum pressure and boson stars shrink at an accelerated pace developing huge boson densities in the center when maximum density reach the critical value $\sim 0.43 / \widetilde{g}^{2}$. Combining the relationship of Eq. 2.24, we know the critical mass of collapse is inversely proportional to $\sqrt{\widetilde{\delta}}$, in accordance with the theoretical critical mass, $M_{c r} \propto 1 /(m \sqrt{g})$ [39.40] (see also in Sec. 2.2]. 

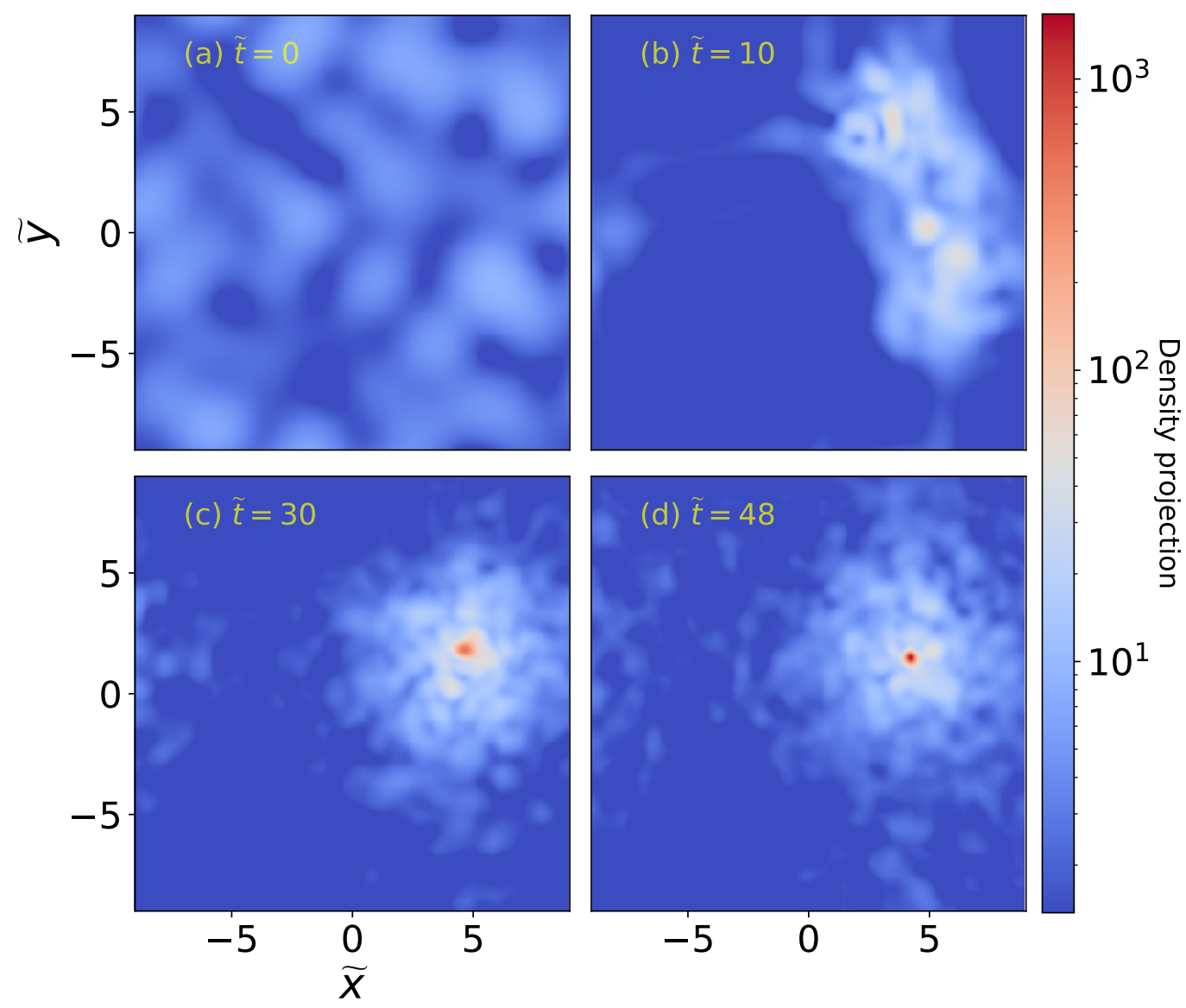

Figure 3.8: Snapshots of the density field from one simulation with $\widetilde{N}=1005.3$, $\widetilde{L}=18, \widetilde{g}=-0.007$. (a) Projected density at the initial time. (b) Projected density at $\widetilde{t}=10$, which shows that minicluster is forming in the box. (c) Projected density at $\widetilde{t}=30$. (d) Projected density at $\widetilde{t}=48$. Compared with the case without selfinteractions, the boson star formed at the center is denser. 


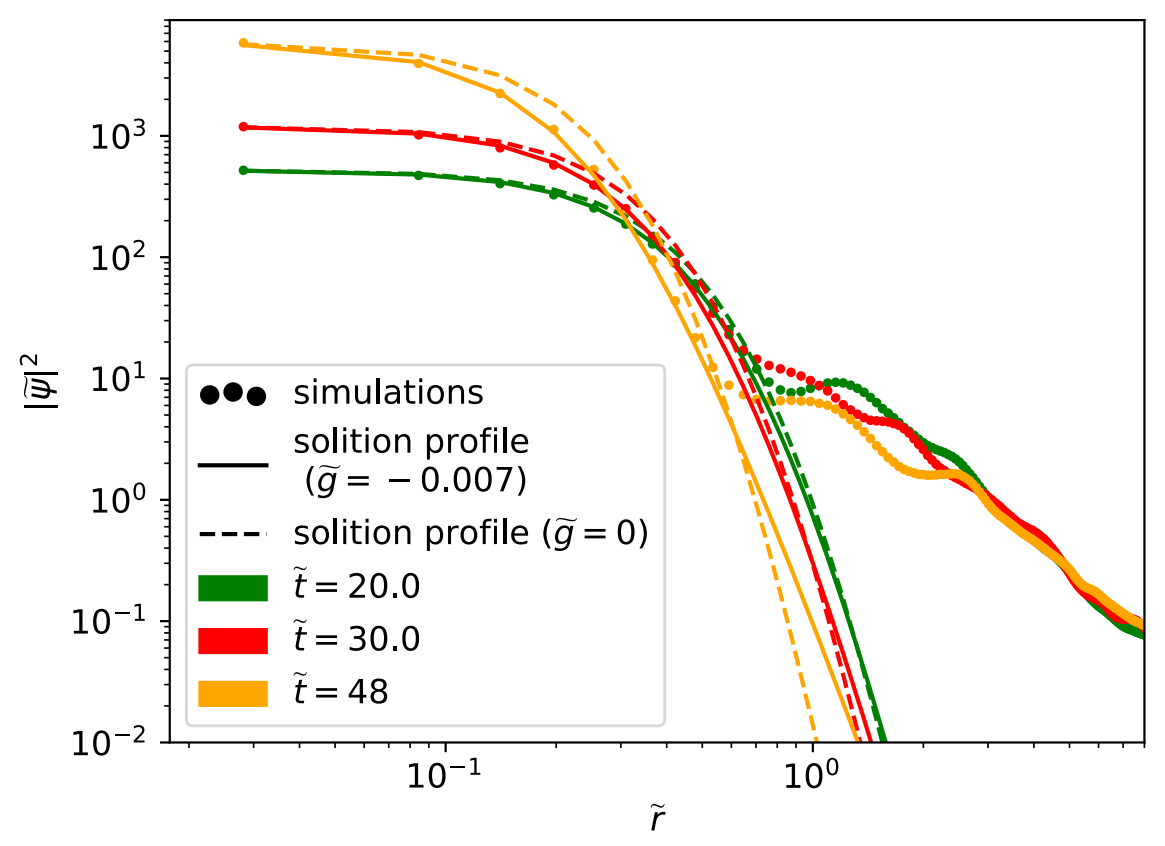

Figure 3.9: Density profiles of the minicluster at different times (colored dots) from simulations of the GPP equations with $\widetilde{g}=-0.007$. Solitonic profiles given by Eq. 2.26 and Eq. 2.25 are plotted in solid and dashed lines, respectively.

\subsubsection{Bosons with repulsive self-interactions}

In this subsection, we study the evolution of some other candidates for dark matter, bosons with repulsive self-interactions ${ }^{2}$.

By simulating the GPP equations with different positive values of $\widetilde{g}$ in a box of size $\widetilde{L}=18$ and total mass $\widetilde{N}=1005.3$, we find miniclusters form and dense objects appear in the center of the miniclusters for sufficiently weak $\tilde{g}$, see Fig. 3.11(a-c).

The density profiles of the dense objects in the cases with $\widetilde{g}=0.01$ and $\widetilde{g}=0.1$ are shown in Fig. 3.12, which can be well fit by the density profiles of solitons given by Eq.2.26. Thus, we confirm that solitons are condensed in the minicluster.

The evolution of maximum density with repulsive self-interactions is shown in Fig. ??. The evolution of the maximum density with $\widetilde{g}=0.01$ coincides with the case without self-interaction $(\widetilde{g}=0)$ at early times. This is similar to the case with weak attractive self-interactions. However, at later times when $\widetilde{t}>\widetilde{\tau}_{\text {sat }}$, the growth rate of the maximum density diverges from the case with no self interactions. The growth rate decreases with increasing $\widetilde{g}$ as expected.

\footnotetext{
${ }^{2}$ The linear theory of bosons with repulsive self-interactions, and constraints on the allowed interaction strength of dark matter, are studied in Ref. [36].
} 


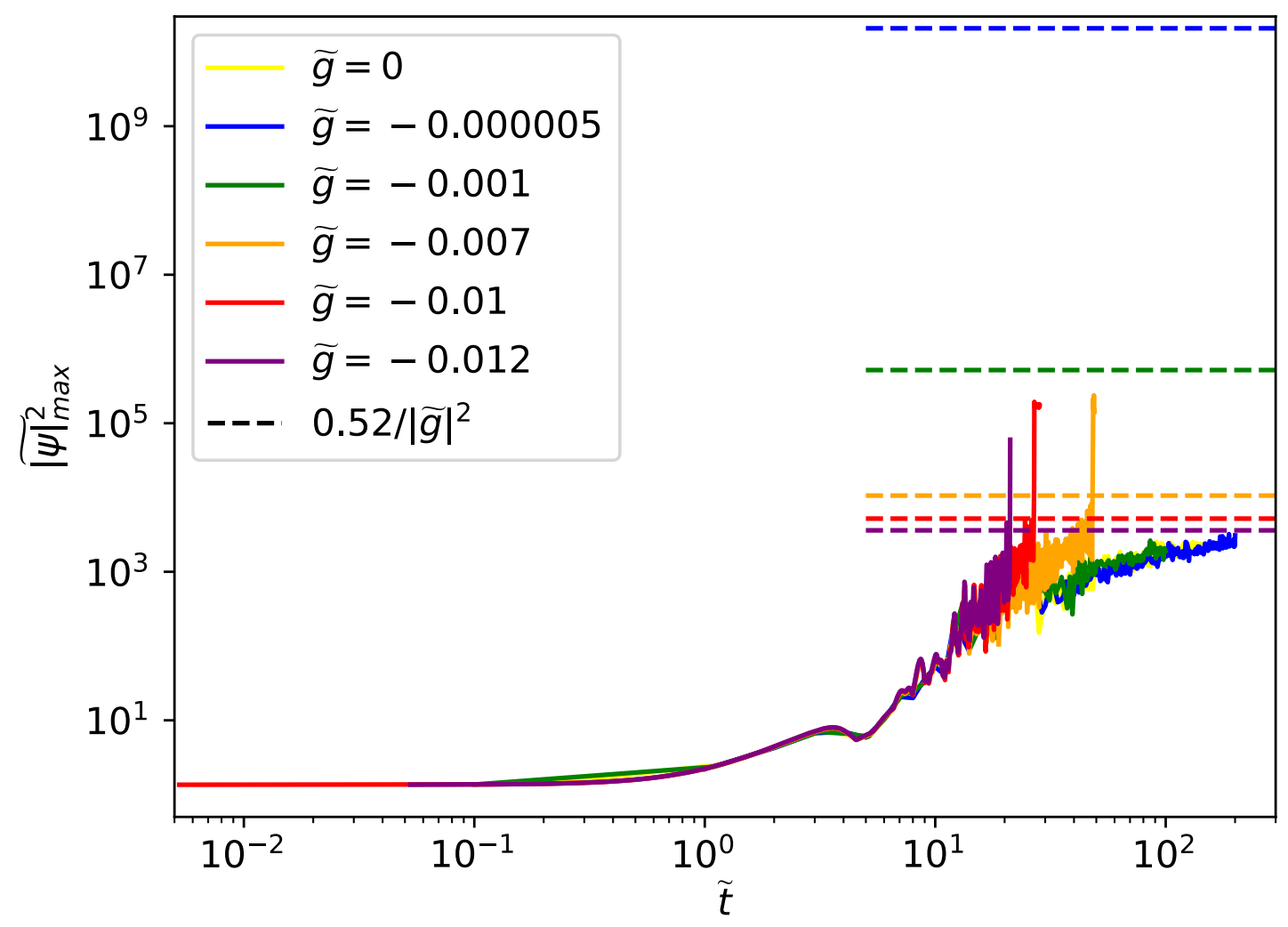

Figure 3.10: Maximum density growth with respect to time from simulations assuming different self-interaction couplings: $\widetilde{g}=0$ (without self-interactions), $\widetilde{g}=-0.000005, \widetilde{g}=-0.001, \widetilde{g}=-0.007, \widetilde{g}=-0.01$, and $\widetilde{g}=-0.012$. The box size $\widetilde{L}=18$ and the total mass $\widetilde{N}=1005.3$. The horizontal dashed lines mark the densities which satisfy $\widetilde{\rho} \widetilde{g}^{2}=0.52$, when the boson star will collapse. 

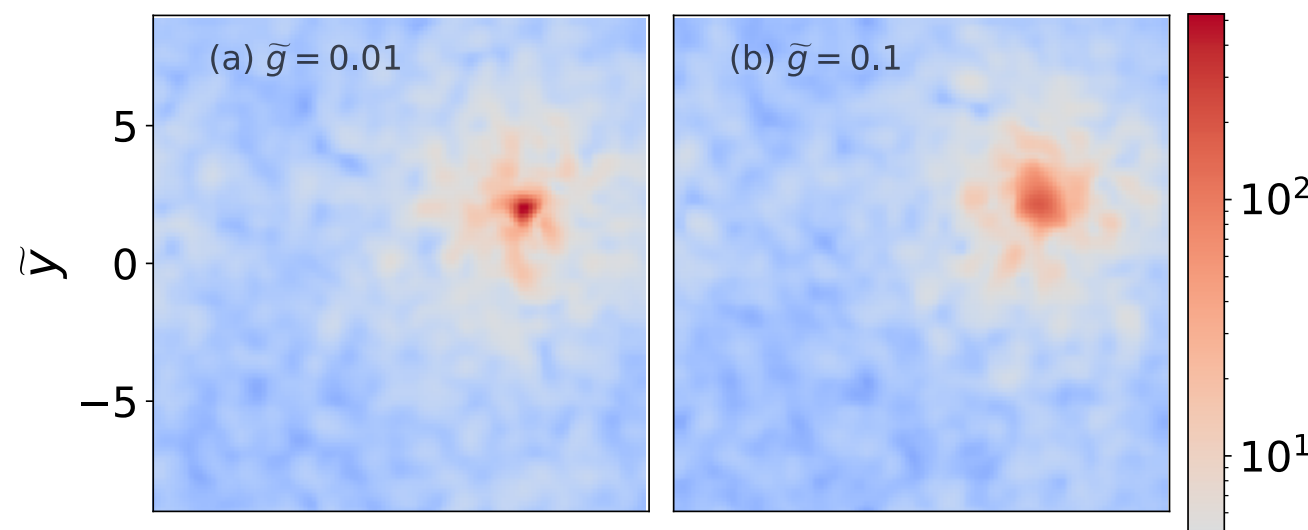

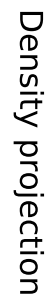

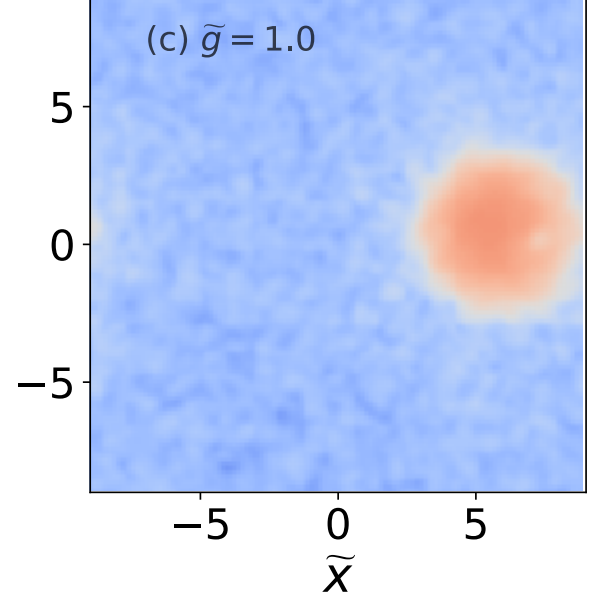

(d) $\widetilde{g}=10.0$

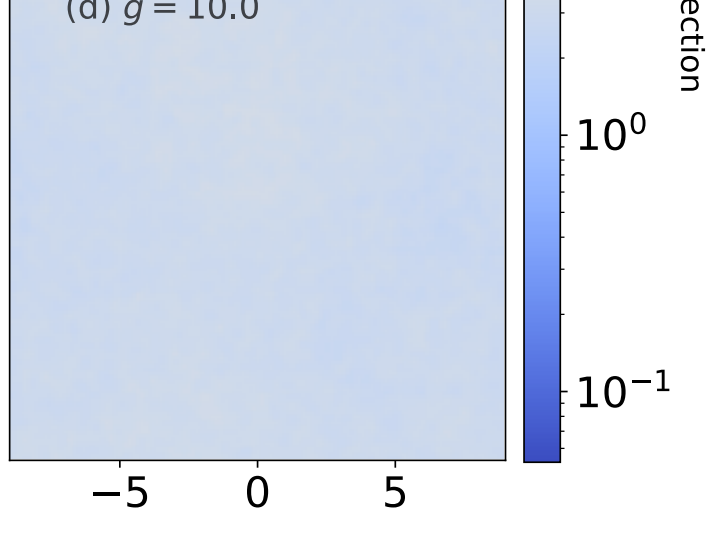

Figure 3.11: Snapshots of the density field at $\widetilde{t}=200$ from simulations in a box of size $\widetilde{L}=18$ and total mass $\widetilde{N}=1005.3$ assuming different $\widetilde{g}$. 


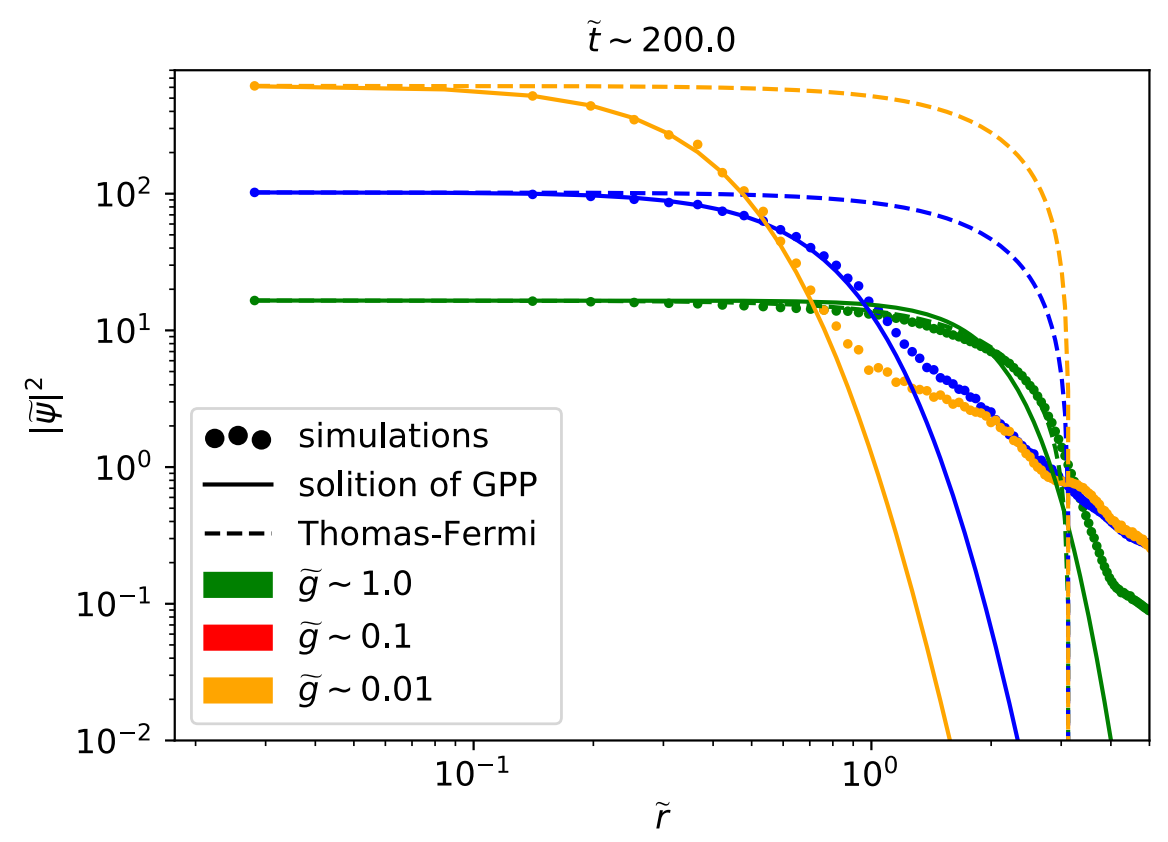

Figure 3.12: Density profiles of the miniclusters from simulations (colored dots), compared with solitonic profiles (solid lines) as given by Eq. 2.25 and ThomasFermi approximation [108] [Eq. (2.31)] with the same central densities.

However, with repulsive self-interactions, the radius of the boson star is larger compared to the case with no self-interactions (see Fig. 3.12). Thus for boson stars with the same central density, the mass of the ones with repulsive self-interactions is larger. To quantify how many particles condense in different cases, we look at the mass growth of boson stars, see the left panel of Fig. 3.13. We find that while the central density growth is slower for larger positive $g$ as shown in the right panel of Fig. 3.13, the mass growth of boson stars is actually faster with increasing $g$ indicating that repulsive self-interactions promote the condensation process.

\subsection{Formation of multiple boson stars}

It is possible that bosons can have even larger values of attractive self-coupling. Thus studying the evolution of these bosons are necessary as well. For bosons with attractive self-interactions, we have shown in Sec. 3.1 that when $|\widetilde{g}|$ is very small, gravity dominates the early stage evolution in systems, and leads to the 

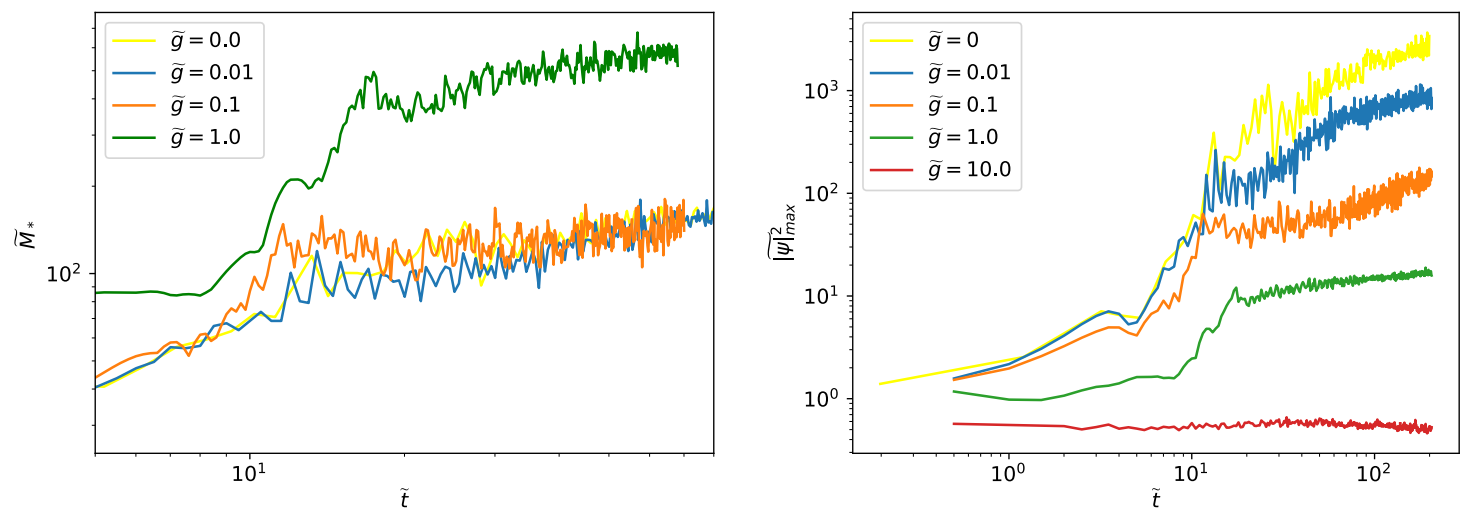

Figure 3.13: Left: Mass growth of boson stars with respect to time from simulations assuming different $\widetilde{g}$ (Note that the case with $g=10$ is not shown since no boson star was formed). Right: Maximum density growth with respect to time from simulations assuming different $\widetilde{g}$. The box size and total mass were $\widetilde{L}=18$ and $\widetilde{N}=1005.3$ respectively.

formation of a single boson star per box ${ }^{3}$ The situation can be very different if $|g|$ is very large, and self-interactions dominate the early stages of evolution [11]. In order to analyze these systems, we can use Eq. 4.2. Firstly, the quantum potential in Eq. 4.2 can be given by

$$
\begin{aligned}
Q & =-\frac{1}{2} \frac{\nabla^{2} \sqrt{\rho}}{\sqrt{\rho}} \\
& =-\frac{1}{2} \frac{\nabla^{2} \sqrt{1+\delta}}{\sqrt{1+\delta}} \approx-\frac{1}{4} \nabla \delta\left(\nabla^{2} \delta\right)
\end{aligned}
$$

and

$$
\nabla h=-\nabla\left(|\psi|^{2}-n\right)=-\rho \nabla_{x} \delta
$$

where $\rho$ is the mean density of systems, $\delta$ is overdensity of systems. Substituting these expressions into Eq. 4.2, we can introduce the governing equation for linear overdensity $\delta \equiv \delta \rho / \rho$. In Fourier space, the linear overdensity $\delta_{k}$ satisfies [54]

$$
\ddot{\delta}_{k}=-\left(\frac{k^{4}}{4 m^{2}}+\frac{g \rho k^{2}}{m^{2}}-4 \pi G \rho\right) \delta_{k} .
$$

Here we have neglected the Hubble friction term and assumed the cosmic scale factor varies slowly on time scales we are concerned with so that it can be treated

\footnotetext{
${ }^{3}$ In cosmological simulations [21, 155, 182], one boson star forms in each halo as it separates out from the cosmic expansion during gravitational collapse. We have verified that this occurs also in our simulations with an expanding background spacetime.
} 
as a constant. It is then easy to show that $\delta_{k}$ will grow exponentially when

$$
k_{J}^{2}<-2 \rho g+2 \sqrt{\rho\left(\rho g^{2}+4 \pi G m^{2}\right)},
$$

i.e. the growth of the linear perturbation is unstable, thus the overdense regions will quickly undergo nonlinear collapse.

The instability scale $k_{J}$ is determined by the strength of gravity and self-interactions. For different values of $g$ and $\rho$, we have:

- $\rho g^{2} \ll 4 m^{2} \pi G$. Gravity dominates, miniclusters form first. After that, one boson star forms in the center of each minicluster.

- $\rho g^{2} \approx 4 m^{2} \pi G$. Gravity and self-interactions both play important roles. A gravitational bound minicluster may contain multiple boson stars formed from local overdensities.

- $\rho g^{2} \gg 4 m^{2} \pi G$. Self-interactions dominate. The condensate can fragment and form multiple boson stars due to self-interactions before a gravitational bound object forms.

To test this hypothesis, we run simulations with very strong attractive selfcouplings. For comparison, we also simulate the GP equations ignoring gravity under the same initial conditions.

Fig. 3.14 shows the evolution of the system simulated using GPP equations with $\widetilde{g}=-0.04$. We can see the formation of a minicluster, Fig. 3.14 (a-c). After that, several boson stars form in the system, Fig. 3.14(d).

Fig. 3.15 (a) and (b) show the systems simulated using GPP equations and GP equations with $\widetilde{g}=-1.0$ at $\widetilde{t}=1.0$. We can see two boson stars condense in the dense areas in Fig. 3.15(a), but not in (b), suggesting that the gravity can promote the condensation of boson stars slightly even when self-interactions are strong.

Fig. 3.15 (c) and (d) show the cases with $\widetilde{g}=-80$. Comparing results from GPP equations with the ones from the GP equations, we don't find a big difference. Therefore, we conclude that the self-interactions dominate the evolution of boson stars alone in some extreme systems.

In fact, Eq. 3.12 shows the self-interactions can be ignored if $-0.53 \lesssim \widetilde{g}<0$ for a system with box size $\widetilde{L}=18$, total mass $\widetilde{N}=1005.3$, and characteristic velocity $\widetilde{v} \sim 1$. But our simulation shows self-interactions are important even for $\widetilde{g}=-0.04$ at the late stages of evolution (see Fig. 3.14(c) and (d)). We think the reason is that 
at these times, the characteristic velocity increases due to gravitational collapse making the gravitational condensation less efficient.

In a cosmological setting, the extreme condensate fragmentation observed in our simulations caused by strong self-interactions would spoil the hierarchical nature of cosmic structure formation. However, these results could be applicable to fragmentation of the inflation condensate (e.g. Ref. [130]) or to condensates in condensed matter.

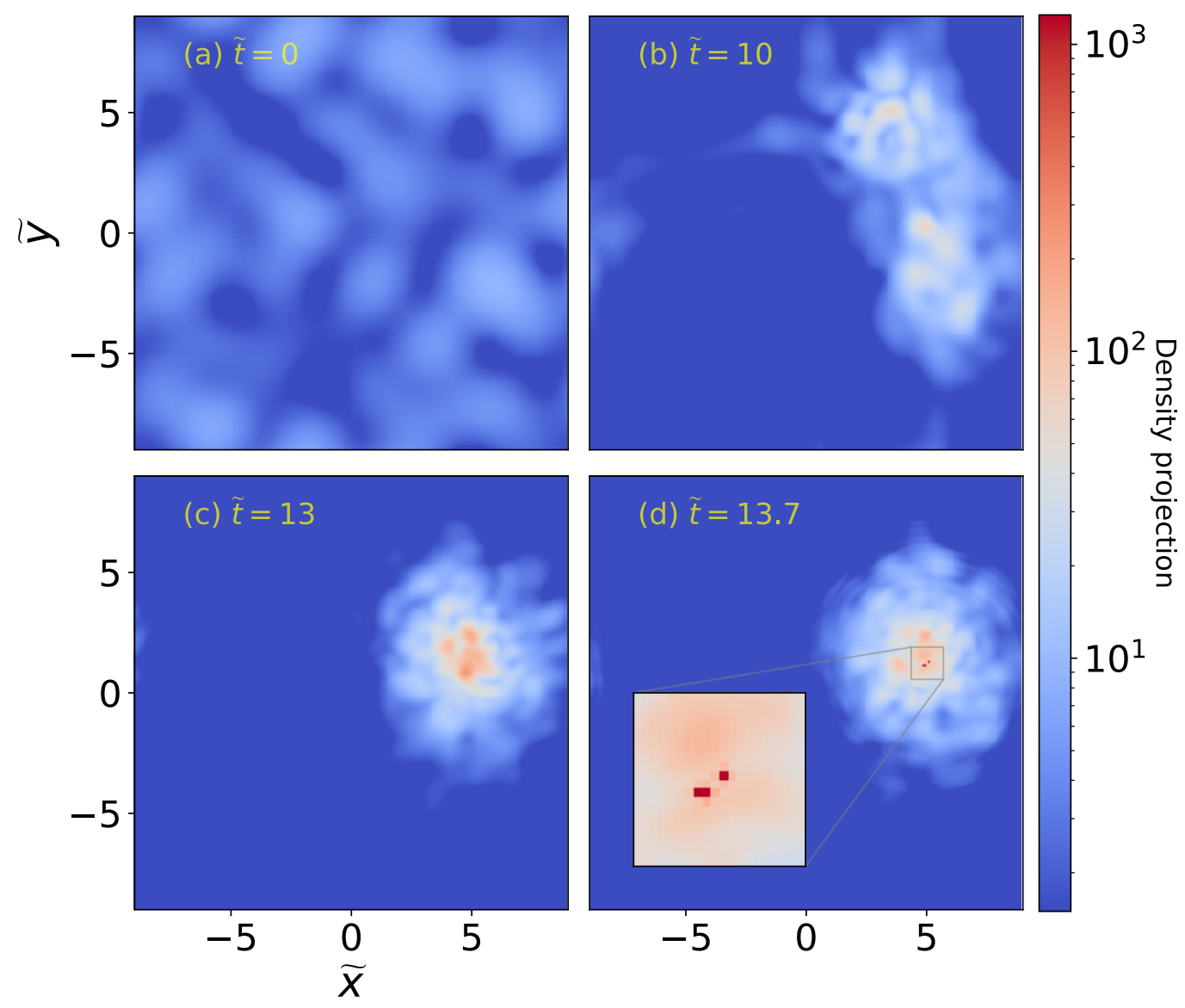

Figure 3.14: Snapshots of the density field from simulations of the GPP equations in a box of size $\widetilde{L}=18$, and total mass $\widetilde{N}=1005.3$. The self-interaction coupling constant $\widetilde{g}=-0.04$. Note that due to resolution limit, we can not resolve the central region of the densest object, so we cutoff the projected density in the plot at $\approx 1500$. 

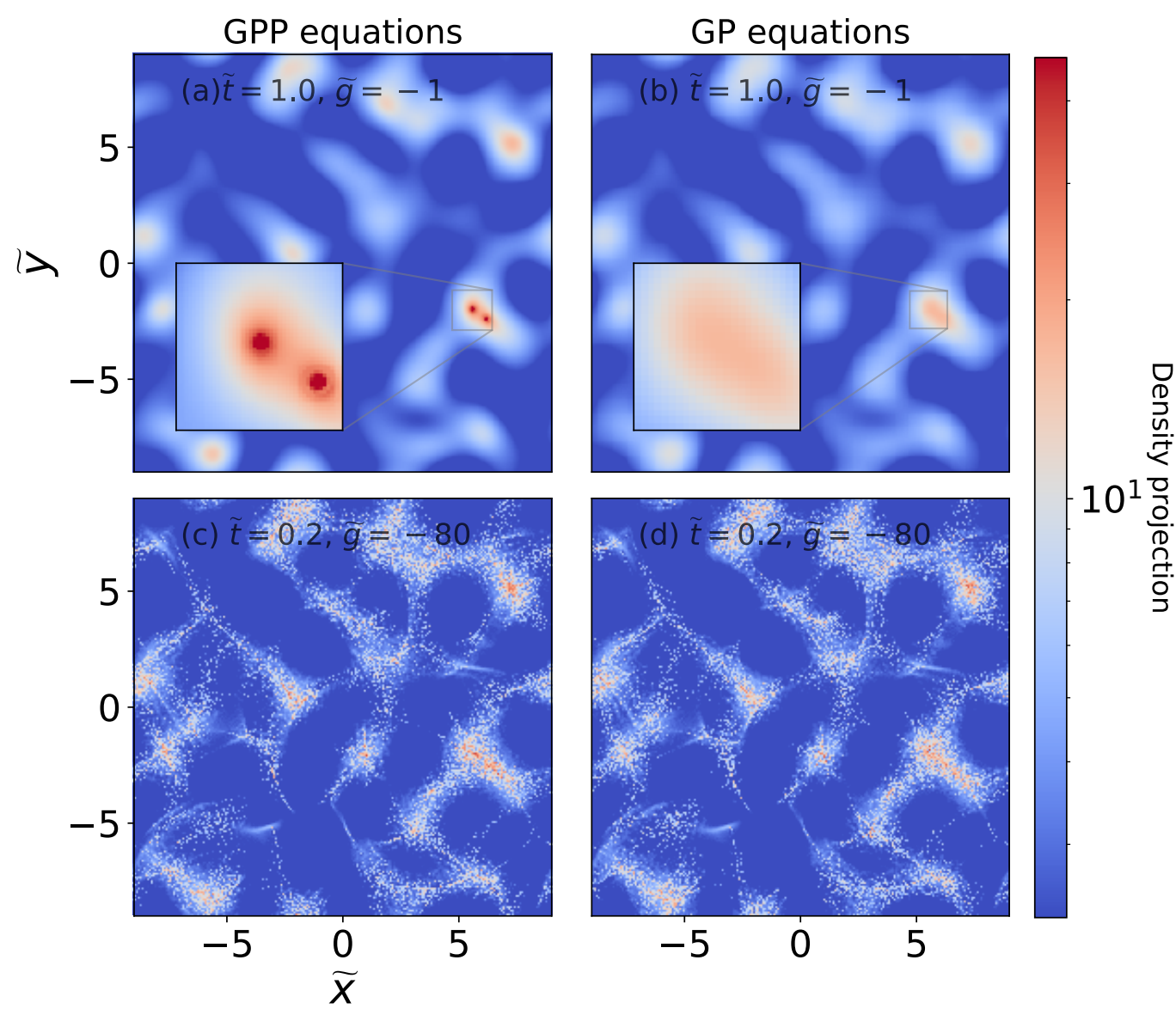

Figure 3.15: Snapshots of the density field from simulations of the GPP equations (left column) and GP equations (right column). We pick $\widetilde{g}=-1$ (first row) and $\widetilde{g}=-80$ (second row). The box size $\widetilde{L}=18$, and the total mass $\widetilde{N}=1005.3$. 


\subsection{Conclusions}

By means of numerical solution of the dynamical Gross-Pitaevskii-Poisson equations, we studied the formation and subsequent growth of boson stars inside gravitationally self-bound halos. We demonstrated a series of new phenomena in the solutions, which had not been seen before in the dynamical regime. Our simulations are local, not cosmological, and so our conclusions apply in all cosmological models that possess the correct environments.

In the case with no self-interactions beyond gravity, we demonstrated the saturation of boson star growth. We ran simulations for times long compared to the dynamical timescales, i.e. $t \gg \tau_{\text {sat }}>\tau_{\text {gravity, }}$ and much longer than those of Ref. [99]. In this regime of boson stars we observed a transition from relatively fast mass growth, $\propto t^{1 / 2}$, to much slower growth, $\propto t^{1 / 8}$, in accordance with the prediction made by Ref. [60]. We attribute this to the formation of a gravitationally bound and virialised atmosphere around the boson star, suppressing further mass growth by coupling the condensation time to the boson star's virial temperature.

Another interesting phenomenon is that we discover no significant difference for the end stage evolution of mass of boson stars (see Fig. 3.6 normalized by $\widetilde{N}^{\alpha} \propto \tilde{M}_{\text {halo }}^{\alpha}$. The best-fit value of $\alpha$ is 0.45 , which is broadly consistent with the core mass-halo mass relation found in previous cosmological simulations, i.e. $\alpha=$ $1 / 3$ [155].

In any case, our observation of a reduced boson star growth rate at late times explains why boson stars in virialised halos in cosmological simulations (e.g. Refs. [60, 154, 182]) are only observed to grow very slowly compared to the other gravitational timescales, and thus populate an almost constant in time core-halo mass relation (see also Ref. [58], which considers the effect of mergers). The saturation of boson star growth will play a role in fixing the cosmological mass function of boson stars formed of axions and ALPs in all cosmological scenarios, although we leave a quantitative study of this to future work.

Our results in the case of attractive self-interactions demonstrated for the first time that boson stars can grow via accretion and reach the critical mass for collapse Once the critical mass is reached, relativistic simulations are needed. The relativistic simulations of Refs. [77, 98] began with super-critical stars, and showed that these stars lead to either ejection of relativistic bosons and a massive remnant (nova) if $f_{a} \lesssim M_{\mathrm{pl}}$, or, for weak self-interactions, $f_{a} \gtrsim M_{\mathrm{pl}}$, collapse to black holes. Our dynamical simulations show that it is possible to reach such critical nova state 
dynamically before saturation. This implies that such a star could undergo a series of novae in its lifetime. This could have implications for the abundance of relativistic particles in the Universe. If the bosons produced can be converted into visible photons, as is the case for axions and axion-like particles, the nova ejecta could even be observed. We leave for future work the study of he expected rates in realistic models. The regime of weak coupling is applicable to the QCD axion, and so we have demonstrated that such novae could occur for models where the QCD axion composes the dark matter. However, further study is required to determine in which astrophysical environments or cosmologies QCD axion novae are expected to occur in abundance.

In the case of very strong attractive interactions, we demonstrated that these can dominate over gravity and lead to fragmentation of the condensate into many small, dense regions. Such fragmentation has not been seen before in simulations including gravity. This has implications for the fragmentation of the inflaton condensate during the reheating epoch [125, 130].

Our results in the case of repulsive interaction demonstrated that such an interaction can promote boson star formation. We showed for the first time that the stable Thomas-Fermi-like solution, which has been studied often in the literature on scalar field DM (e.g. Refs. [108]), can be reached dynamically via gravitational accretion. Repulsive self-interactions change the mass-radius relation of boson stars, and we have shown that these solitons can also be formed dynamically via condensation. A realisitic formation mechanism for such states also has implications for the gravitational wave searches for exotic compact objects [70], and could be used to predict the expected signal rates in gravitational wave detectors [45]. Given the sign and strength of the interaction required in this case, the formation of ThomasFermi condensates is not applicable to the QCD axion, but could occur in more generalised ALP or scalar field dark matter models with strong attractive self interactions.

In summary, we have demonstrated new results on the dynamical formation and growth of boson stars in a collection of different models, including self-gravity, attractive and repulsive self-interactions. Our results have applications to future terrestrial, astrophysical, and cosmological observations searching for new types of bosons across a wide range of scales. 


\section{Chapter 4}

\section{Turbluence creation in the process of merger of boson stars}

In fluid dynamics, turbulence is a particular type of fluid motion. It is characterised by the chaotic changes which occur in the flow velocity and pressure. Turbulent flows are distinctly different from another type of fluid motion known as laminar flow. The laminar flow is that the fluid flows in layers which don't mix with each other when the flow velocity is very small. [23].

Turbulence is a very common phenomenon in nature: the flow of water through a pipe, the exhaust gas discharged from a factory chimney or coffee being stirred by a spoon. All of these phenomena demonstrate turbulence. Fig. 4.1 is a diagrammatic sketch which shows the transition from laminar flow to turbulence flow.

The key characteristics of turbulence is below: The first feature is their irregularity. As such, we can only describe the turbulence statistically as opposed to deterministically. The second feature of turbulence is its diffusivity. Turbulence can increase the rate of energy density transportation in a fluid. Therefore, the fluid is able to reach thermal equilibrium faster. The third key feature of turbulence is dissipation. Through viscous shear stress, kinetic energy within the fluid is converted into internal energy. This makes the turbulence dissipate. The rate of dissipation depends on the intensity of viscous shear stress. The forth feature is rotationality. Turbulence possesses non-zero vorticity and powerful three-dimensional vortex generation mechanism. This is so-called vortex stretching. Generally speaking, Due to volume conservation of fluid element, vortex stretching can make vortex perpendicular to the stretching direction much thinner. This leads to the decomposition of larger flow structure into smaller structure. This process will continue until the kinetic energy of structure can be converted into heat by molecular viscosity of the fluid. 


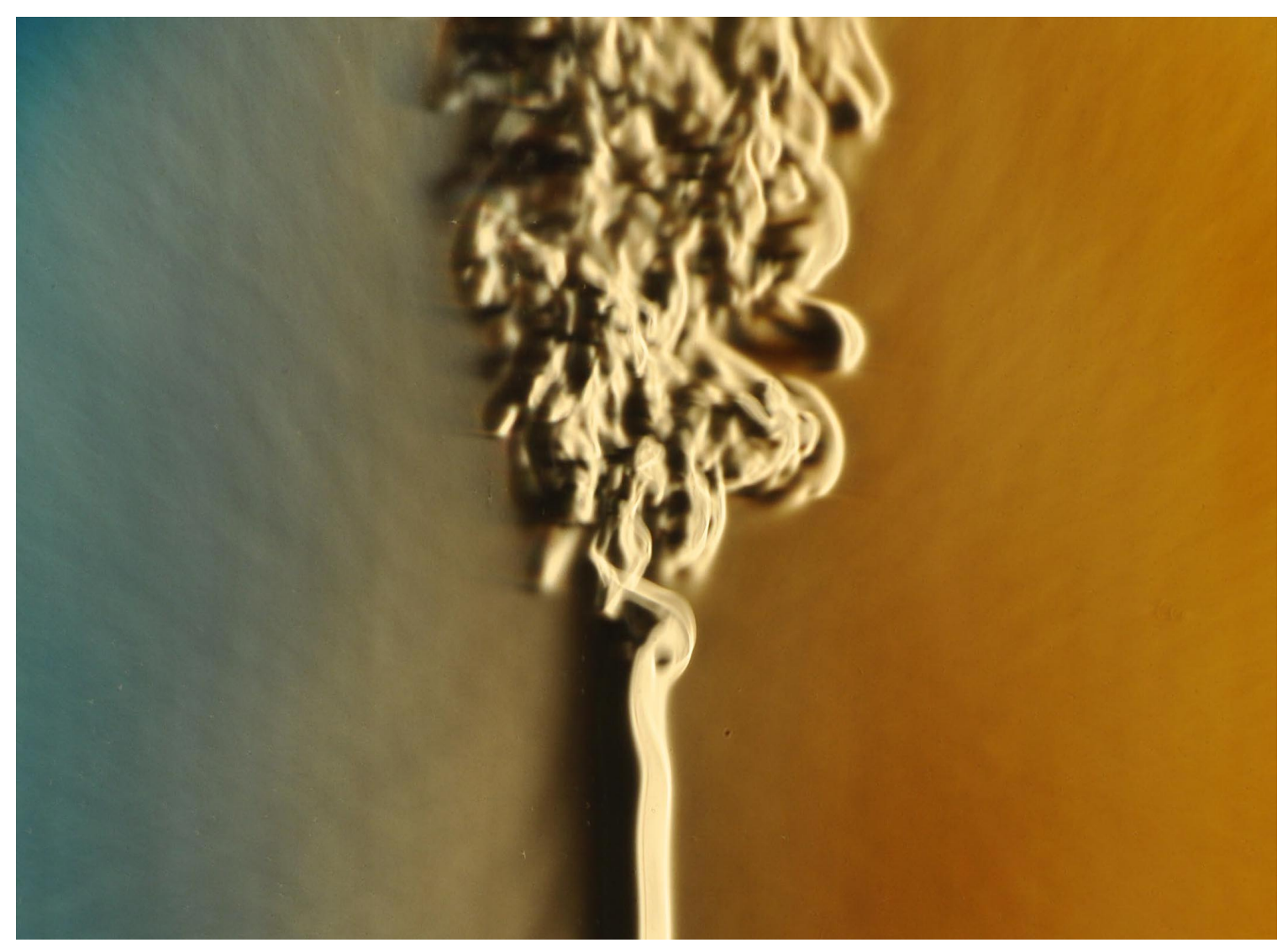

Figure 4.1: The transition from the laminar flow to turbulence flow. Reproduced from [31]. 
Under most common conditions, all fluids experience flow resistance, called viscosity, which controls the transition from laminar to turbulent flow and causes the turbulence to decay. For example, after stirring a cup of coffee, it will eventually return to rest. However, we know that there exist a special class of fluids known as superfluids [1], which were first discovered in liquid helium by John F. Allen and Pyotr Kapitsa in 1937. The key feature of this fluid is that it has no viscosity or flow resistance, which means that the flow around a closed circuit will continue forever.

However, turbulence still can occur within a superfluid despite the lack of viscosity. This was first proposed theoretically by Richard Feynman in 1955 [63] and was quickly confirmed through experiments [166].

Based on what is known as the Kolmogorov turbulence cascade scenario [131], one feature of turbulence is the existence of coherent structures, which are longstanding phenomena in turbulence. The coherent structure may even have correlations between different length scales that differ greatly. The latter property indicates local scale coupling. Quantum vortices can be considered as the existence of coherent objects in quantum turbulence.

Due to the the nonlinearity in turbulence, we use numerical methods to examine quantum turbulence by vortex creation and annihilation using the concept from the case of classical fluid turbulence [43]. We investigate the case in which ultralight bosons are dominated by gravity. Ultra-light bosons can be seen as a quantum superfluid since no viscous force between particles exists. We know that turbulence can make the mass distribution of a fluid uniform. In addition, thermal equilibrium will be faster. Thus the research of quantum turbulence of ultra-light bosons can help us to understand more deeply the mass distribution and evolution in our Universe. We used a high-precision algorithm to study the generation of turbulence in the merger of several boson stars (all bosons are in a condensed state), and successfully verified the results by Mocz et al. [124]. After that, we extended to the cases including self-interactions, and found that turbulence also exists in this case.

\subsection{Fluid Formulation}

We begin with the fluid equations,

$$
\dot{\rho}+\nabla(\rho \mathbf{v})=0
$$




$$
\begin{gathered}
\dot{\mathbf{v}}+\mathbf{v} \nabla \mathbf{v}=-\nabla V+\frac{1}{2 m^{2}} \nabla\left(\frac{\nabla^{2} \sqrt{\rho}}{\sqrt{\rho}}\right)-\frac{g}{\rho} \nabla \frac{\rho^{2}}{2}, \\
\nabla^{2} V=4 \pi G m\left(\rho^{2}-n\right) .
\end{gathered}
$$

For bosons with only gravity, we can set $g=0$. Naively, we might assume that vortices can not exist since the velocity field is a gradient flow, $\nabla \times v=\nabla \times \nabla S=$ 0 . However, the phase $S$ is undefined at positions with $\rho=0$. Thus vortices can appear and rotational flows set in when $S$ is a multi-valued function. If density $\rho$ vanishes and the quantum potential term, $\frac{\nabla^{2} \sqrt{\rho}}{\sqrt{\rho}}$ diverges, $S$ will occur multivalued. Thus vortices always exist in the area where $|\psi|^{2} \rightarrow 0$ [43], i.e. locations of destructive interference, which occur generically for a complex wavefunction.

\subsubsection{Power spectrum of turbulence}

The superfluid energy spectrum $E_{v^{2}}(k)$ is given by

$$
E_{v^{2}}=\frac{1}{L_{\text {box }}^{3}} \int \frac{1}{2}|\mathbf{v}|^{2} d \mathbf{x}=\int E(k) d k .
$$

where $L_{\mathrm{box}}$ is the box side length [19]. For the mechanically driven turbulence in classical fluid, we have a classical Kolmogorov scaling $E(k) \propto k^{-5 / 3}$, which was first proposed by Andrei N. Kolmogorov by dimensional analysis in 1941. We define $L^{\prime}$ to be the length dimension, $T^{\prime}$ is time dimension and $M^{\prime}$ is mass dimension. The energy dimension is then

$$
E^{\prime}=\frac{M^{\prime} L^{\prime}}{T^{\prime 2}}
$$

It should be noted that $E(k)$ in Kolmogorov's theory is not energy, but the kinetic energy per unit wave number range and per unit mass of matter. The total energy per unit mass needs to be obtained by integrating $E(k)$ over the wave number. The wave number is defined as $k=\frac{2 \pi}{L}$. This corresponds to a dimension of $k^{\prime}=\frac{1}{L^{\prime}}$. Therefore, the dimension of $E(k)$ is

$$
E(k)=\frac{L^{\prime 3}}{T^{\prime 2}}
$$

The energy flow per unit time and per unit mass has dimensions:

$$
\epsilon=\frac{L^{\prime 2}}{T^{\prime 3}}
$$


Because energy is transferred step by step in turbulence, a reasonable assumption is that the energy on a certain scale is related to the scale and energy flow, that is

$$
E(k) \propto \epsilon^{a} k^{b} .
$$

Through dimensional analysis, it is not very difficult to obtain $a=\frac{2}{3}, b=-\frac{5}{3}$. According to the self similarity of turbulence, Kolmogorov theory assumes that $\epsilon$ is constant on all scales. So the Kolmogorov scaling is

$$
E(k) \propto k^{-5 / 3} \text {. }
$$

However, the situation for quantum superfluid is different. The power spectrum is proportional to $k^{-1}$ rather than $k^{-5 / 3}$ since local flows around the vortex lines dominate the fluid velocity. We can derive the asymptotic behavior of the power spectrum from the spectrum of one vortex. The transverse velocity around a vortex $\left|v_{\perp}\right|$ scales as $|r|^{-1}$. Therefore, the Fourier component, $\left|v_{k}\right| \propto k^{-1}$, which mean that the spectrum is proportional to $k^{-1}$. If the vortices in systems are only weakly correlated, the total spectrum can be obtained by summing all vortex spectra. So the power spectrum is still proportional to $k^{-1}$ at small scales. In addition, since the vortex loop occupies a region of measure zero, the zero-separation contribution dominates the vortex-vortex two-point correlation function. Therefore, the power spectrum still keeps as $k^{-1}$.

These theoretical prediction have been proved by Tzihong Chiueh et al firstly [43]. They consider evolution of free bosons. Through solving the time-dependent, Schrödinger equation, $i \frac{\partial}{\partial t} \psi=-\frac{1}{2 m} \nabla^{2} \psi$, evolving from a random-phased initial distribution, they found vortex creation, annihilation and interactions in quantum turbulence. Furthermore, they found that the energy power spectrum of quantum turbulence is proportional to $k^{-1}$ in small scales [43].

\subsection{Turbulence in merges of boson stars}

In 2017, Mocz et al. extended results of Tzihong Chiueh et al to scalar particles with gravity. They found the vortex creation in the process of mergers of boson stars with gravity using a second order Pseudo-Spectral method [124]. In our study, we begin by verifying these results using higher precision methods since we have implemented the higher precision fourth-order method, see Sec 2.6.3. On the other hand, many candidate particles of ultra-light bosons own weak attractive 
self-interaction, such as the QCD axions. In Section 3.3, we showed that such selfinteractions can play an important role in the dynamics. Therefore, in this chapter, we investigate the effect of self-interactions on vortex creation and turbulence.

\subsubsection{Merger of boson stars}

We begin by creating a gravitationally bound DM halo from the merger of a number of boson stars. Numerically solving the SP equations at box size $30<\widetilde{L}<100$ and number of boson stars $5<N_{\text {star }}<20$, we observe the merger of boson stars and its surrounding halo/minicluster. One example is shown in the left panel of Fig 4.2. box size $\widetilde{L}=40$ and 10 same boson stars, total mass $N=266$ at different positions. We can see the boson stars, see the left panel of Fig 4.2(a). We can see a boson stars merge gradually from $\widetilde{t} \approx 10$ to $\widetilde{t} \approx 30$. A dense core exist in the center of halo, granule-like structure with similar size as the core is seen all round the halo, see the left panel of Fig 4.2(d). We find that the radial density profile of the minicluster from this most dense point coincides with the density profile of a soliton solution at $0<\widetilde{r}<1$ (soliton density profiles are described in Eq.2.25), see the right panel of Fig 4.2 . These results are fully consistent with results of Refs. [?, 155].

On the other hand, we study the merger of boson stars with weak attractive self interaction. For the convenience of comparison, we also simulated 10 boson stars with the same quality in box size $\widetilde{L}=40$ and total mass $N=266$. Their initial positions are the same place as the case without self interaction. Comparing Fig 4.2(d) and Fig 4.3(d), we still can see the merger of boson stars and core occur in the box, i.e. Here we set $\widetilde{g}=-0.007$.

\subsubsection{Turbulence in halos/miniclusters}

For bosons with gravity, our simulations provide strong evidence of existence of turbulence everywhere due to identification of vortex lines, located at $|\psi| \rightarrow 0$, see left panel of Fig. 4.4. These filamentary structures are source of turbulence, which has been proved in Sec. 4.1. The middle panel of Fig. 4.4 shows the phase of wavefunction phase, $S$. Near the filamentary structure, we can see that $\sin S$ drops from $\approx 1$ to $\approx-1$. This indicates the discontinuities of $S$. The right panel of Fig. 4.4 is 3D volume rendering of vortex line, which also illustrate vortex lines exist everywhere in the box. Vortex line reconnection arises from vortex collision. Fig 4.5 show details of vortex line reconnection. In addition, the turbulent structure exist everywhere except inside boson stars. 

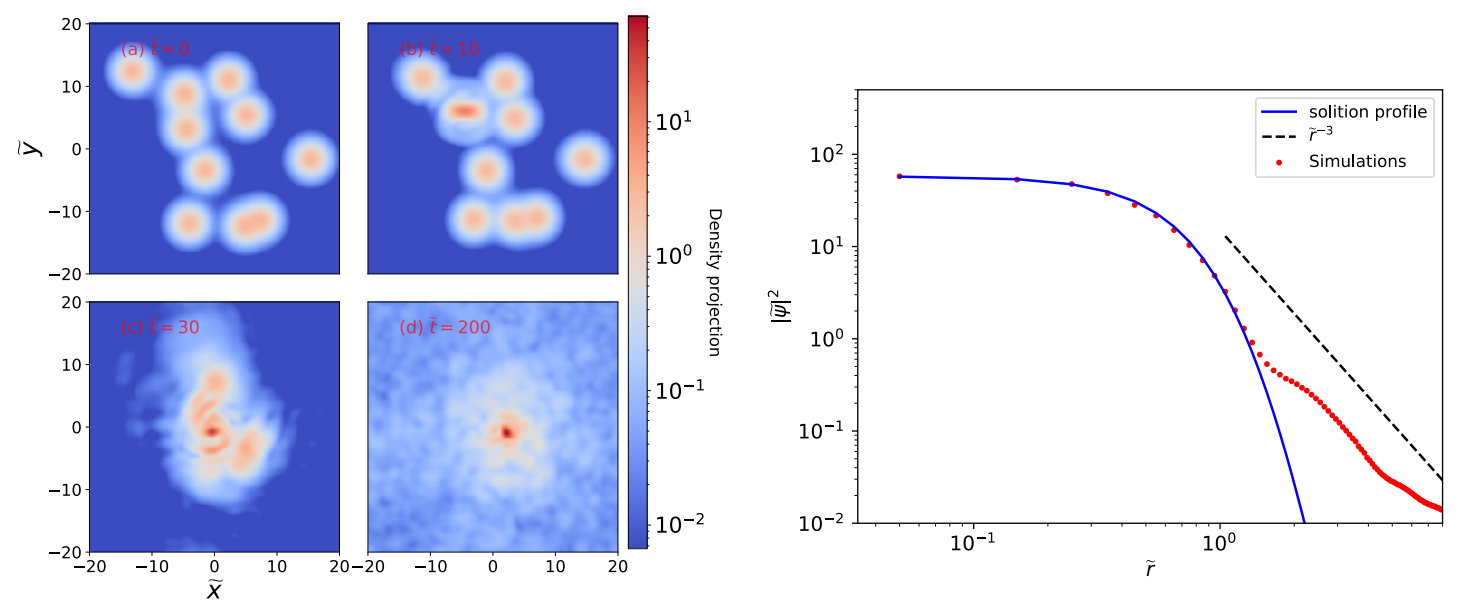

Figure 4.2: The left panel is snapshots of the density field from one simulation with 10 same boson stars in different positions. The box size $\widetilde{L}=40$ and the total mass $\widetilde{N}=258$. (a) Projected density at the initial time. (b) (c) Projected density at $\widetilde{t}=10$ and $\widetilde{t}=30$, which shows several boson star has merged together. (d) Projected density at $\widetilde{t}=200$. All boson star meger together. A single dense object is visible at the centre of the dark matter halo. The right panel is density profiles of the minicluster at $\widetilde{t}=200$ (colored dots) compared with solitonic profiles (blue lines) as given by Eq. 2.25 with the same central densities.
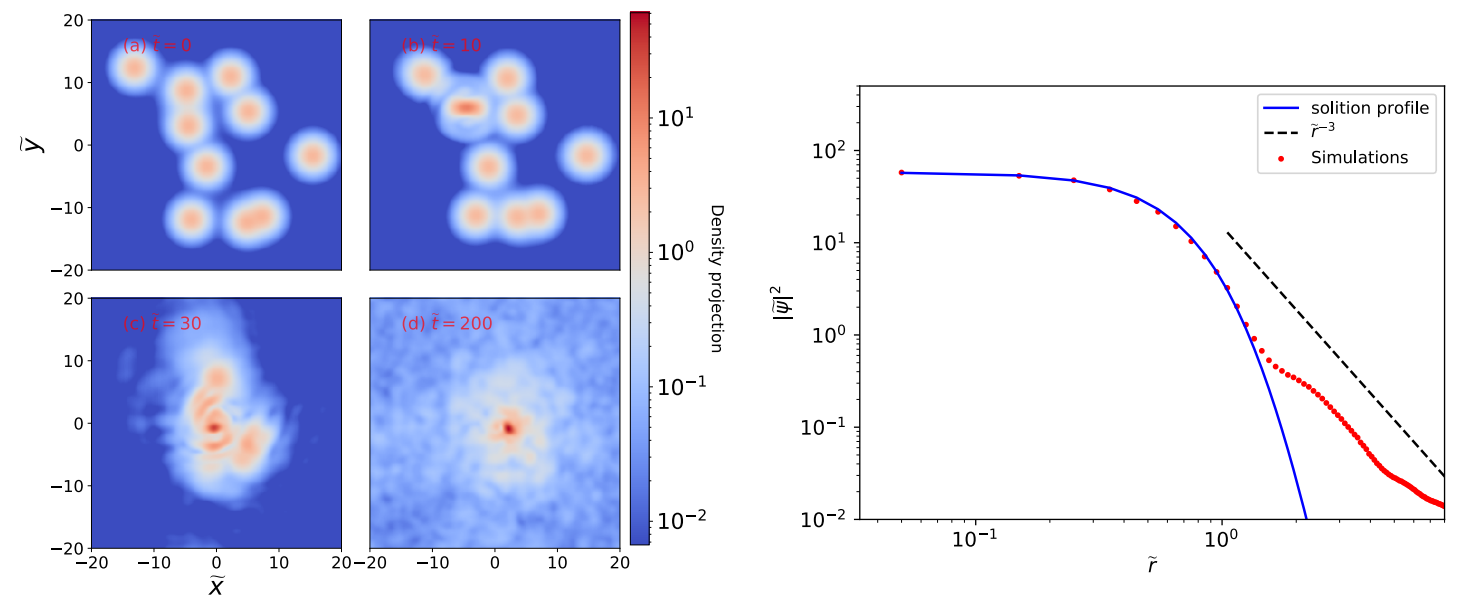

Figure 4.3: The left panel shows Snapshots of the density field from one simulation with 10 same boson stars. The box size $\widetilde{L}=40$ and the total mass $\widetilde{N}=258$. (a) Projected density at the initial time. (b) (c) Projected density at $\widetilde{t}=10$ and $\widetilde{t}=30$, which shows several boson star has merged together. (d) Projected density at $\widetilde{t}=200$. The right panel shows the density profile of the minicluster at $\widetilde{t}=200$ (colored dots) compared with solitonic profile, $\widetilde{g}=-0.007$, (blue lines) with the same central densities. 
The left panel of Fig. 4.6 shows the energy power spectrum of our simulations. The simulations show $E_{v^{2}}(k)$ is proportional to $k^{-1}$ rather than $k^{-5 / 3}$ at large $k$. The system is more akin to a thermally-driven counterflow analog Gross-Pitaevskii system than one which is mechanical driven. The right panel of Fig 4.6 confirms this conclusion statistically as we vary number of boson star at 10, 15, 20 and total mass, $\widetilde{N}$ within the range $[200,1000]$.
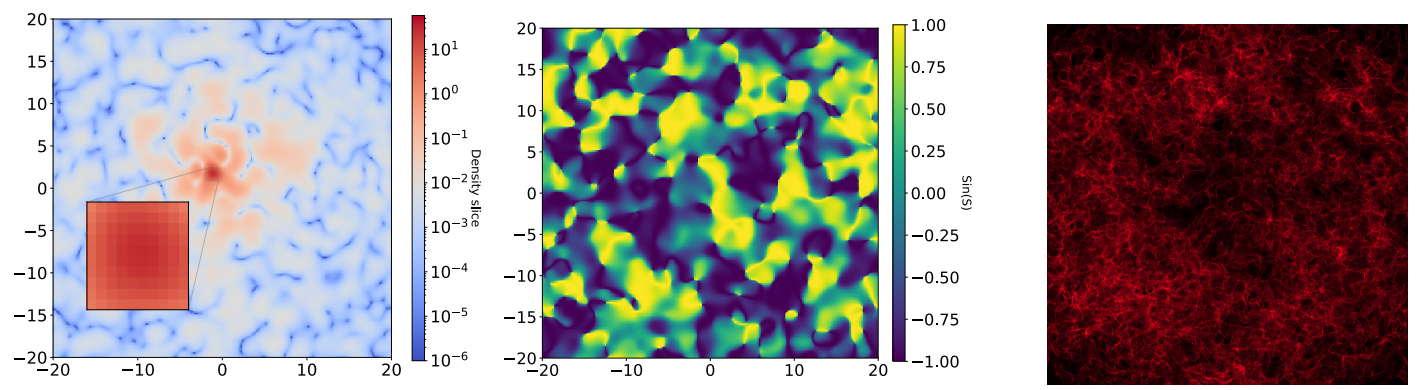

Figure 4.4: The left panel is the density slice of the minicluster at $\widetilde{t}=200$. The middle panel shows the wavefunction phase $S$ at $\widetilde{t}=200$. The right panel is $3 \mathrm{D}$ volume rendering of the vortex line at $\widetilde{t}=200$. The box size $\widetilde{L}=40$ and the total mass $\widetilde{N}=258$.
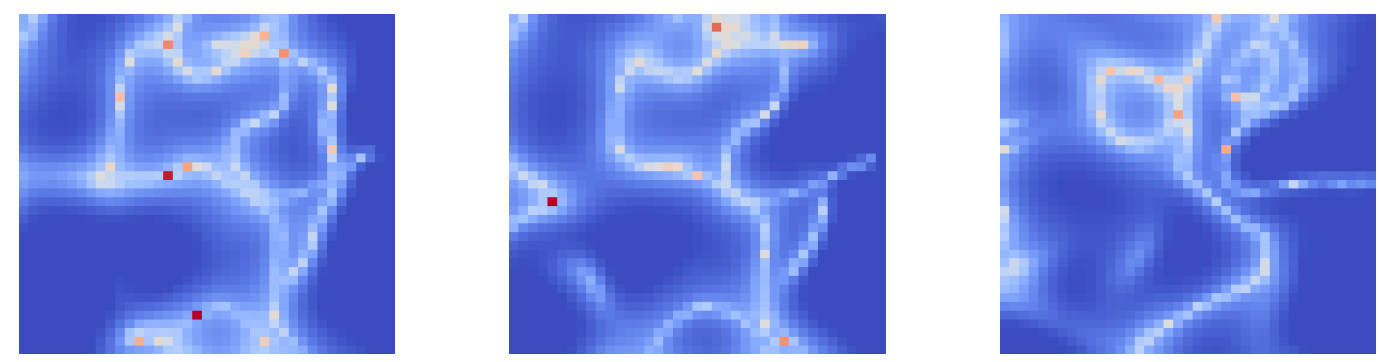

Figure 4.5: Zooming-in projection of $1 /|\psi|^{2}$ for bosons with gravity. The annihilation of vortex line are shown.

For bosons with both gravity and weak attractive self-interaction, we run simulation from initial condition with same total mass and initial position of boson stars. Compared with no self-interaction case, we find no significant difference visually in the turbulence. The vortex lines, located at $|\psi| \rightarrow 0$, still exist everywhere. Vortex line reconnection also can be found, see Fig 4.8 . Thus the weak attractive self interaction can be ignored in the creation of turbulence.

On the other hand, we also found that the distribution of phases is different, which shows that self interaction has an impact as during the merger process of boson stars, but the discontinuity of the phase we focus on still exists. In other words, turbulence still exists. 

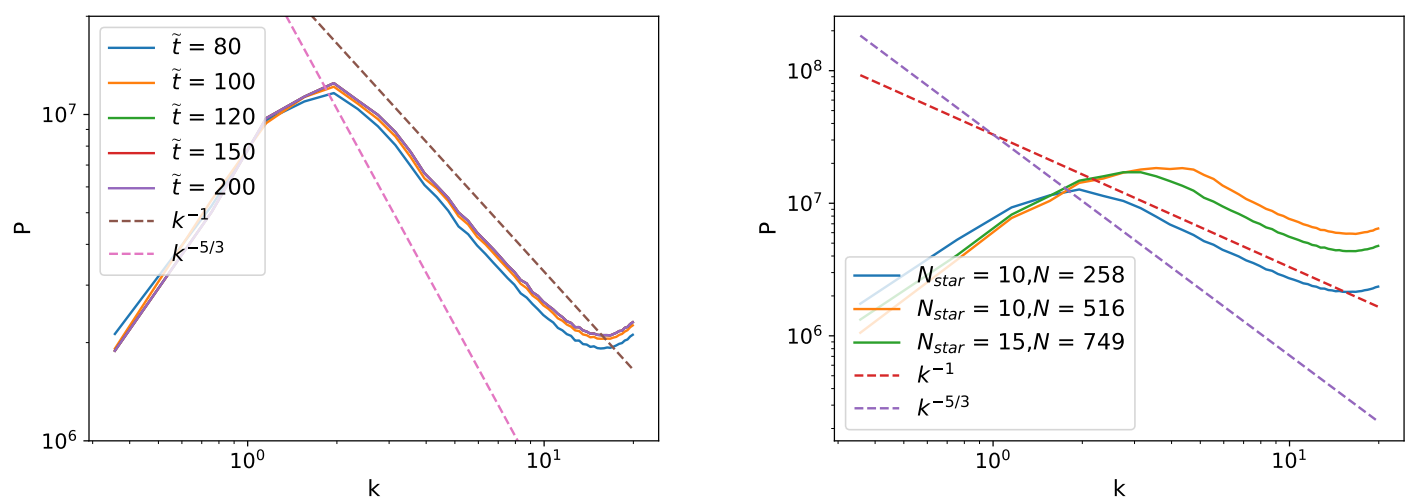

Figure 4.6: The left panel is the velocity power spectra at different times after the merger of 10 same boson stars for boson with only gravity. Here we set the box size $\widetilde{L}=40$ and the total mass $\widetilde{N}=258$. The right panel shows the velocity power spectra from different initial conditions. Here we choose several results with different number of boson stars and total mass, $\widetilde{N}$.

The left panel of Fig. 4.9 also supports this conclusion. The energy power spectrum $E_{v^{2}}(k)$ approaches to $k^{-1}$ at large $k$ at different time. The right panel of Fig. 4.9 shows the energy power spectrum for bosons with different self-coupling coefficient $\widetilde{g}$, from -0.007 to 0.07 . We can still see that $E_{v^{2}}(k)$ approaches to $k^{-1}$.
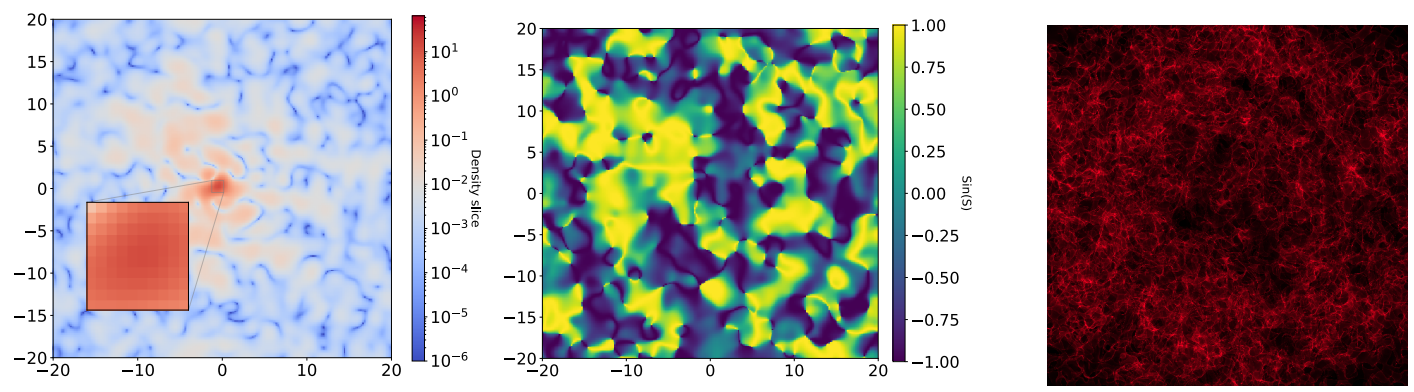

Figure 4.7: The left panel is the density slice of the minicluster at $\widetilde{t}=200$. The middle panel is the wavefunction phase $S$ at $\widetilde{t}=200$. The right panel is 3D volume rendering of the vortex line at $\widetilde{t}=200$. Here we set $\widetilde{g}=-0.007$, the box size $\widetilde{L}=40$ and the total mass $\widetilde{N}=258$.

\subsubsection{Comparison between second-order and fourth-order algo- rithms}

Mocz et al. used the second-order algorithm in time integration, and we have a higher-precision fourth-order algorithm. Here we compare two numerical algorithms. We find that the total energy is fourth-order conserved as expected in 

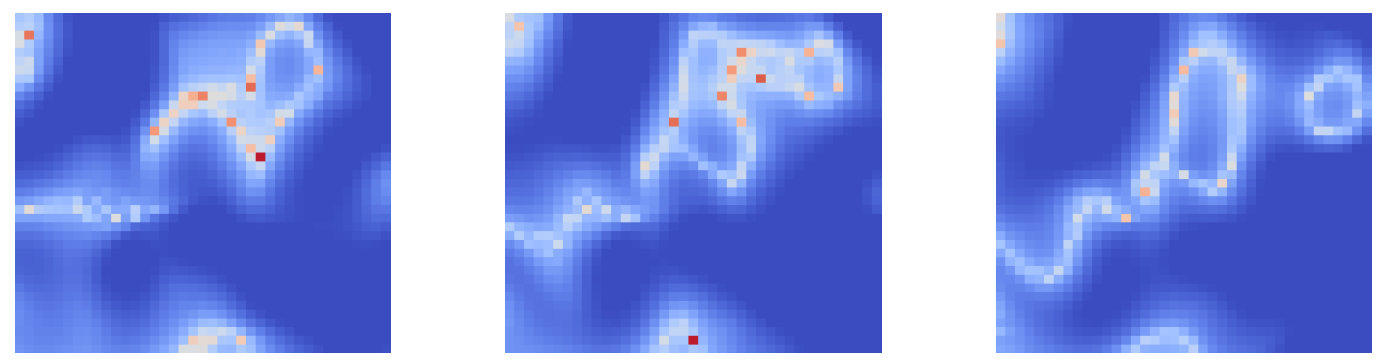

Figure 4.8: Zooming-in projection of $1 /|\psi|^{2}$ for bosons with gravity and weak attractive self-interaction. We can see the annihilation of vortex line. Here, we set $\widetilde{g}=-0.007$.
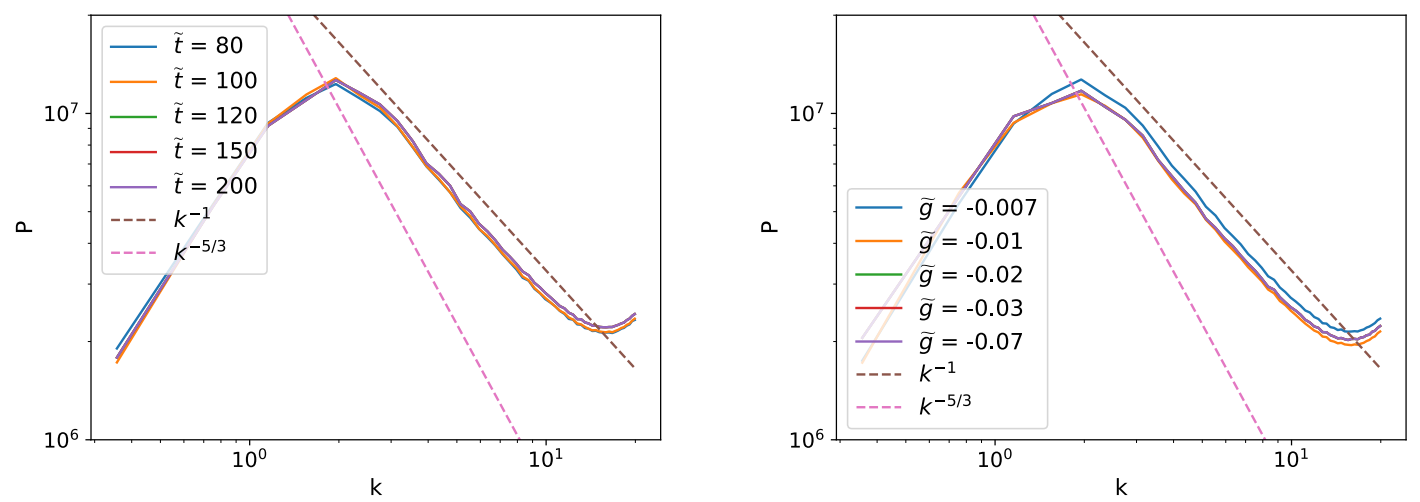

Figure 4.9: The left panel is the velocity power spectra of merger of 10 same boson stars at different time. Here we set $\widetilde{g}=-0.007$, the box size $\widetilde{L}=40$ and the total mass $\widetilde{N}=258$. The right panel is velocity power spectra of merger of 10 same boson stars for different $\widetilde{g}$. Here we set the box size $\widetilde{L}=40$, all of boson stars with same center density, $\rho_{c}=1.01$. 
out fourth-order algorithm. We have confirmed of Mocz et al's results through a higher-precision algorithm. As shown in Fig. 4.10, despite that the time step size is $\pi$ times larger, the fourth-order algorithm has better accuracy by several orders of magnitudes.
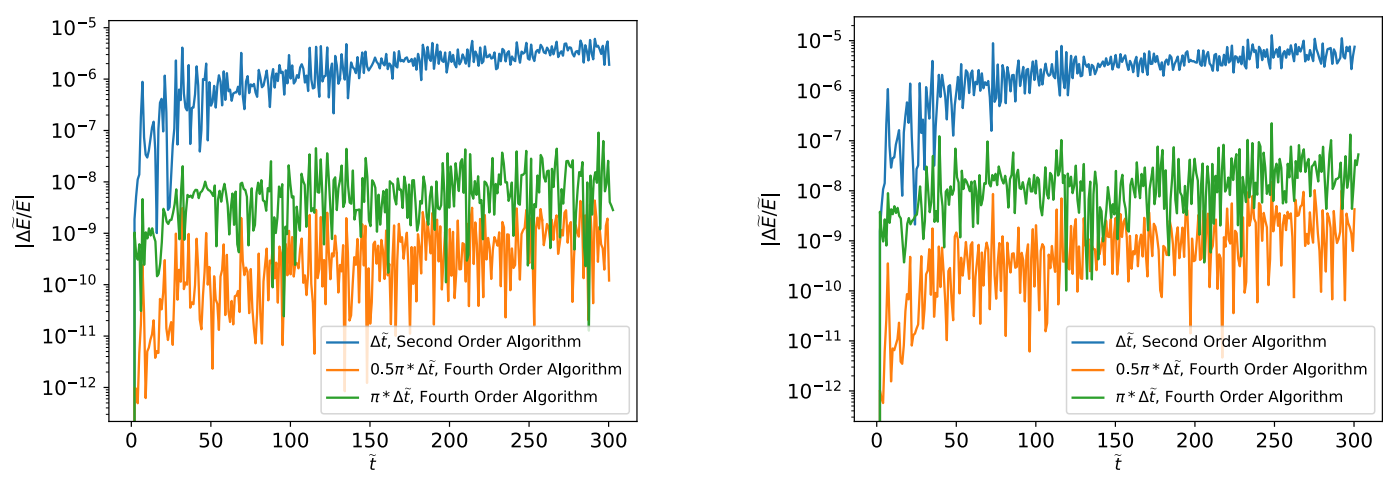

Figure 4.10: Relative errors of the total energy with respect to time for different algorithms. The left panel is the evolution of bosons with no self-interaction, the right panel is the evolution of bosons with weak self-interaction, $\widetilde{g}=-0.007$. 


\subsection{Conclusion}

Quantum turbulence is a relative new research field compared to the conventional turbulence in viscous fluids. The existence of turbulence can cause a fluid to reach thermal equilibrium, as well as impacting the mass distribution in the fluid. Since viscous forces do not exist between ultra-light bosons, they can be treated as the quantum superfluid. Thus it is important to understand the behaviour of turbulence in fields composed of ultralight bosons.

In order to understand the vortex creation and evolution in quantum turbulence, Tzihong Chiueh solved the time-dependent solutions of the free- particle Schrödinger equation in a periodic box of $1024^{3}$ grids for three dimensions in 2011 [43]. They successfully found the reconnection of vortex loop. In addition, $E_{v^{2}}(k)$ is proportional to $k^{-1}$ at small scales.

Based on these results, Mocz et al considered the system of bosons with gravity and found similar phenomenon to the process of merger of boson stars. I repeat their simulations in higher order algorithm to verify their conclusions.

Due to the nonlinearity of quantum turbulence, we need to use numerical methods to model its evolution. Using fourth-order pseudo-spectral method, we study mergers of multiple boson stars, which finally form haloes and soliton cores at their center.

For bosons with only gravitational interactions, we confirm the conclusion of Mocz et al. in Ref. [124] that turbulence is generated in the process of merger of multiply boson stars. We run simulations with a higher order pseudo-spectral method, i.e. forth order compared to the second order method employed by Mocz et al. We found that no turbulence exist within the solitons, $0<\widetilde{r}<1$. The turbulence can be found beyond this radius, $\widetilde{r}>1$ in the haloes. Due to interference, lines with zero density appear in the superfluid composed of bosons. The discontinuities of wavefunction phase $S$ exist in these systems where $\rho \sim 0$. All of these phenomena support the existence of turbulence in the haloes. On the other hand, by checking the power spectrum, we find that these systems are more similar to a thermally-driven counterflow analog Gross-Pitaevskii system since $E_{v^{2}}(k)$ is proportional to $k^{-1}$ at small scales.

After considering bosons with gravity, we try to extend the research about bosons with gravity and weak attractive self-interaction. We find no turbulence exist within the soliton. However, we can see the existence of lines of vanishing density in the haloes. The discontinuities of wavefunction phase $S$ can also be found in 
such systems. Furthermore, we find that the power spectrum of systems $E_{v^{2}}(k)$ is proportional to $k^{-1}$ as well. This means turbulence is a very common phenomenon in a superfluid composed of bosons with gravity and weak self-interactions.

Unfortunately, due to limitations in computing power, we are unable to simulate the merger of boson stars with strong self-interactions. As we saw in Sec. 3.3. boson stars will collapse after they reach a critical mass. Levkov et al. also observed an emission of relativistic bosons after boson stars collapse [100]. Therefore, it is valuable research topic whether this phenomenon can affect turbulence in halos. 


\section{Chapter 5}

\section{Outlook}

In this dissertation, we briefly introduce the history of dark matter. Our research focuses on one of the popular candidates of dark matter, ultra-light bosons, especially the ground states of ultra-light bosons, non-compact boson stars. We write a high precision numerical code, employing the pseudo-spectral method, to solve GPP equations. With GPU acceleration, the codes are speed-up by 6-7 times compared to the pure CPU code. Using these codes, we confirm the results by Argelia Bernal et al., colliding of two boson stars [25], and Hsi-Yu Schive et al., merger of multiply boson stars [155].

After that, we obtain new results about the formation and growth of boson stars in dark matter halos [41]. Firstly, for ultralight bosons without self-interaction, the saturation of boson stars occurs in miniclusters. The mass growth rate of boson stars drops from $\propto t^{1 / 2}$ to $\propto t^{1 / 8}$ as conjectured by [60]. Secondly, for bosons with attractive self-interaction, such as QCD axions $\left(10^{-20} \mathrm{GeV}^{-2}<g<10^{-35} \mathrm{GeV}^{-2}\right)$, the self-interaction can cause collapse of boson stars above a critical mass. However, it does not affect condensation and early-stage evolution of boson stars in miniclusters. Thirdly, for bosons with a repulsive self-interaction, condensation and growth of boson stars is promoted. At strong coupling, the resulting boson stars are well described by the Thomas-Fermi profile [108], with a larger radius than the case with no self-interaction. Fourthly, for strong attractive selfinteractions, the condensate can fragment and form multiple boson stars even in a small simulation box (see also [11] for the case with a saturated scalar potential).

We also check the vortex creation in the process of merger of boson stars with gravity. The results show that turbulence exist every places in halos except inside the boson stars. Similar phenomenon exist for boson stars with gravity and weak attractive self-interaction. 
One possible extension of my current work in the future is studying formation and evolution of boson stars in cosmological background. So far I have focused on the the condensation of bosons in non-cosmological background. It will be interesting to look at how the expanding cosmological background affects the condensation process and the saturation behaviors of boson stars. The study will be crucial to estimating the abundance of boson stars in the universe and the relation between the DM halo mass and its central boson star (solitonic core). For this application, I have already implemented in my pseudo-spectral code the support for an expanding cosmological background. With a large set of simulations in the near future, I will be able to answer the questions mentioned above.

Although the cosmological simulation will be limited by the small volume that can be simulated, I expect to get some qualitatively new results on the formation rate of boson stars. Combining these results with analytic and semi-analytic methods that are used in the research of cosmological structure formation, e.g. the Press-Schechter formalism, I will estimate the abundance and mass distribution of the boson stars in our Universe. By comparing these predictions with observations of the mass distribution in the universe, e.g. from the gravitational lensing and radio astornomy, we may get a constraint on the mass of scalar particle.

Another interest of mine is study the condensation time of boson star due to self-interaction in more detail. In a recent work by Kirkpatrick et al., it has been argued that the relaxation rate due to self-interaction is proportional to $|g|$ rather than $g^{2}$ [93], suggesting a much shorter condensation time due to self-interaction compared to the one reported by other literature. Conducting a larger set of simulations with different coupling constants and initial conditions, I will be able to check whether the new prediction is correct. If it is, it will be interesting to see how the self-interaction may promote the formation of boson star in early universe when the matter density, thus the self-interaction, is much higher.

Studying colliding of two boson stars close to the critical mass is interesting since boson stars in our galaxy could have binary systems. Previous studies showed that boson star with attractive self-interaction collapse at a critical mass. However, when two stable boson stars close to the critical mass collide with certain velocity, do they collapse and emit relativistic bosons. If the emitted relativistic bosons can be converted into visible photons, as is in the case of axions and axion-like particles, the nova ejecta could even be observed.

The effect of strong attractive and repulsive self-interaction for the vortex creation in the process of merger of boson stars can also be studied. Unlike the weak 
attractive self-interaction, strong self-interaction can not be neglected in the systems. The structure of halos and boson stars are different. Our studies show that boson stars with larger radii can be formed for bosons with strong repulsive selfinteraction. For bosons with strong attractive self-interaction, multiply boson stars can be formed in halos [41]. Thus it is worth to study whether these phenomenons can influence vortex creation in systems. 


\section{Bibliography}

[1] Superfluid hydrodynamics, volume 3, January 1974.

[2] B. P. Abbott, R. Abbott, T. D. Abbott, Abernathy, et al. Observation of Gravitational Waves from a Binary Black Hole Merger. , 116(6):061102, February 2016.

[3] L.F. Abbott and P. Sikivie. A Cosmological Bound on the Invisible Axion. Phys. Lett. B, 120:133-136, 1983.

[4] George O. Abell. The distribution of rich clusters of galaxies. PhD thesis, California Institute of Technology, January 1957.

[5] R Acciarri, M Antonello, B Baibussinov, et al. The WArP experiment. Journal of Physics: Conference Series, 203:012006, jan 2010.

[6] G. E. Addison, G. Hinshaw, and M. Halpern. Cosmological constraints from baryon acoustic oscillations and clustering of large-scale structure. Monthly Notices of the Royal Astronomical Society, 436(2):1674-1683, Sep 2013.

[7] P. A. R. Ade, N. Aghanim, M. I. R. Alves, C. Armitage-Caplan, M. Arnaud, M. Ashdown, F. Atrio-Barandela, J. Aumont, H. Aussel, and et al. Planck2013 results. i. overview of products and scientific results. Astronomy Astrophysics, 571:A1, Oct 2014.

[8] M. Aguilar, G. Alberti, B. Alpat, et al. First result from the alpha magnetic spectrometer on the international space station: Precision measurement of the positron fraction in primary cosmic rays of $0.5-350$ gev. Phys. Rev. Lett., 110:141102, Apr 2013.

[9] D. S. Akerib, H. M. Araújo, X. Bai, et al. First results from the lux dark matter experiment at the sanford underground research facility. Phys. Rev. Lett., 112:091303, Mar 2014. 
[10] V. A. Ambartsumian. On the Evolution of Galaxies. 28:241-279, 1958.

[11] Mustafa A. Amin and Philip Mocz. Formation, gravitational clustering, and interactions of nonrelativistic solitons in an expanding universe. Physical Review D, 100(6), Sep 2019.

[12] V. Anastassopoulos, S. Aune, K. Barth, et al. New CAST limit on the axionphoton interaction. Nature Physics, 13(6):584-590, June 2017.

[13] E. Aprile, M. Alfonsi, K. Arisaka, et al. Observation and applications of single-electron charge signals in the XENON100 experiment. Journal of Physics G Nuclear Physics, 41(3):035201, March 2014.

[14] M. Arik, S. Aune, K. Barth, A. Belov, et al. Search for Sub-eV Mass Solar Axions by the CERN Axion Solar Telescope with He3 Buffer Gas. , 107(26):261302, December 2011.

[15] Asimina Arvanitaki, Savas Dimopoulos, Sergei Dubovsky, Nemanja Kaloper, and John March-Russell. String Axiverse. Phys. Rev. D, 81:123530, 2010.

[16] S. J. Asztalos, G. Carosi, C. Hagmann, D. Kinion, K. van Bibber, M. Hotz, L. J. Rosenberg, G. Rybka, J. Hoskins, J. Hwang, P. Sikivie, D. B. Tanner, R. Bradley, J. Clarke, and ADMX Collaboration. SQUID-Based Microwave Cavity Search for Dark-Matter Axions. , 104(4):041301, January 2010.

[17] W. B. Atwood, A. A. Abdo, M. Ackermann, W. Althouse, et al. The Large Area Telescope on the Fermi Gamma-Ray Space Telescope Mission. , 697(2):1071-1102, June 2009.

[18] Horace W. Babcock. The rotation of the Andromeda Nebula. Lick Observatory Bulletin, 498:41-51, January 1939.

[19] Andrew W. Baggaley, Jason Laurie, and Carlo F. Barenghi. Vortex-Density Fluctuations, Energy Spectra, and Vortical Regions in Superfluid Turbulence. , 109(20):205304, November 2012.

[20] C. A. Baker, D. D. Doyle, P. Geltenbort, K. Green, M. G. D. van der Grinten, P. G. Harris, P. Iaydjiev, S. N. Ivanov, D. J. R. May, J. M. Pendlebury, and et al. Improved experimental limit on the electric dipole moment of the neutron. Physical Review Letters, 97(13), Sep 2006. 
[21] Nitsan Bar, Diego Blas, Kfir Blum, and Sergey Sibiryakov. Galactic rotation curves versus ultralight dark matter: Implications of the soliton-host halo relation. Phys. Rev. D, 98(8):083027, 2018.

[22] K. Barth, A. Belov, B. Beltran, H. Bräuninger, et al. CAST constraints on the axion-electron coupling. , 2013(5):010, May 2013.

[23] G. K. Batchelor. An Introduction to Fluid Dynamics. Cambridge Mathematical Library. Cambridge University Press, 2000.

[24] Katrin Becker, Melanie Becker, and John H. Schwarz. String Theory and MTheory. 2007.

[25] Argelia Bernal and F. Siddhartha Guzmán. Scalar field dark matter: Head-on interaction between two structures. Phys. Rev. D, 74:103002, Nov 2006.

[26] Gianfranco Bertone and David Merritt. Dark Matter Dynamics and Indirect Detection. Modern Physics Letters A, 20(14):1021-1036, Jan 2005.

[27] Miguel Bezares and Carlos Palenzuela. Gravitational Waves from Dark Boson Star binary mergers. Class. Quant. Grav., 35(23):234002, 2018.

[28] Michael R. Blanton, Matthew A. Bershady, Bela Abolfathi, et al. Sloan Digital Sky Survey IV: Mapping the Milky Way, Nearby Galaxies, and the Distant Universe. , 154(1):28, July 2017.

[29] S. Boran, S. Desai, E. O. Kahya, and R. P. Woodard. GW170817 falsifies dark matter emulators. , 97(4):041501, February 2018.

[30] M. Born and R. Oppenheimer. Zur Quantentheorie der Molekeln. Annalen der Physik, 389(20):457-484, January 1927.

[31] horst Bronk. Laminar-turbulent transition, 2009.

[32] Dmitry Budker, Peter W. Graham, Micah Ledbetter, Surjeet Rajendran, and Alexander O. Sushkov. Proposal for a Cosmic Axion Spin Precession Experiment (CASPEr). Physical Review X, 4(2):021030, April 2014.

[33] Clare Burrage, Edmund J. Copeland, Christian Käding, and Peter Millington. Symmetron scalar fields: Modified gravity, dark matter, or both? Phys. Rev. D, 99:043539, Feb 2019. 
[34] R. R. Caldwell. A phantom menace? Cosmological consequences of a dark energy component with super-negative equation of state. Physics Letters B, 545(1-2):23-29, October 2002.

[35] P. Candelas, Gary T. Horowitz, Andrew Strominger, and Edward Witten. Vacuum configurations for superstrings. Nuclear Physics B, 258:46-74, January 1985.

[36] J. A. R. Cembranos, A. L. Maroto, S. J. Núñez Jareño, and H. Villarrubia-Rojo. Constraints on anharmonic corrections of fuzzy dark matter. Journal of High Energy Physics, 2018(8), Aug 2018.

[37] Jose A. R. Cembranos. Modified gravity and dark matter. Journal of Physics: Conference Series, 718:032004, may 2016.

[38] P.-H. Chavanis. Mass-radius relation of Newtonian self-gravitating BoseEinstein condensates with short-range interactions. I. Analytical results. , 84(4):043531, August 2011.

[39] P.H. Chavanis and L. Delfini. Mass-radius relation of Newtonian selfgravitating Bose-Einstein condensates with short-range interactions: II. Numerical results. Phys. Rev. D, 84:043532, 2011.

[40] Pierre-Henri Chavanis. Collapse of a self-gravitating Bose-Einstein condensate with attractive self-interaction. Phys. Rev. D, 94(8):083007, 2016.

[41] Jiajun Chen, Xiaolong Du, Erik W. Lentz, David J. E. Marsh, and Jens C. Niemeyer. New insights into the formation and growth of boson stars in dark matter halos. 112020.

[42] Siu A. Chin. Forward and non-forward symplectic integrators in solving classical dynamics problems, 2007.

[43] Tzihong Chiueh, Tak-Pong Woo, Hung-Yu Jian, and Hsi-Yu Schive. Vortex turbulence in linear schrödinger wave mechanics. Journal of Physics B: Atomic, Molecular and Optical Physics, 44(11):115101, May 2011.

[44] Ki-Young Choi, Jihn E. Kim, Hyun Min Lee, and Osamu Seto. Neutralino dark matter from heavy axino decay. Phys. Rev. D, 77:123501, Jun 2008. 
[45] Katy Clough, Tim Dietrich, and Jens C. Niemeyer. Axion star collisions with black holes and neutron stars in full 3D numerical relativity. Phys. Rev. D, 98(8):083020, 2018.

[46] Douglas Clowe, Anthony Gonzalez, and Maxim Markevitch. Weak-Lensing Mass Reconstruction of the Interacting Cluster 1E 0657-558: Direct Evidence for the Existence of Dark Matter. , 604(2):596-603, April 2004.

[47] E. Corbelli and P. Salucci. The extended rotation curve and the dark matter halo of m33. Monthly Notices of the Royal Astronomical Society, 311(2):441-447, Jan 2000.

[48] Edvige Corbelli and Paolo Salucci. The extended rotation curve and the dark matter halo of M33. , 311(2):441-447, January 2000.

[49] R. Cowsik and J. McClelland. An Upper Limit on the Neutrino Rest Mass. , 29(10):669-670, September 1972.

[50] R. J. Crewther, P. Di Vecchia, G. Veneziano, and Edward Witten. Chiral Estimate of the Electric Dipole Moment of the Neutron in Quantum Chromodynamics. Phys. Lett. B, 88:123, 1979. [Erratum: Phys.Lett.B 91, 487 (1980)].

[51] Mariateresa Crosta, Marco Giammaria, Mario G Lattanzi, and Eloisa Poggio. On testing cdm and geometry-driven milky way rotation curve models with gaia dr2. Monthly Notices of the Royal Astronomical Society, 496(2):2107-2122, Jun 2020.

[52] P. de Bernardis, P. A. R. Ade, J. J. Bock, et al. A flat Universe from highresolution maps of the cosmic microwave background radiation. , 404:955959, April 2000.

[53] J. G. de Swart, G. Bertone, and J. van Dongen. How dark matter came to matter. Nature Astronomy, 1:0059, March 2017.

[54] Vincent Desjacques, Alex Kehagias, and Antonio Riotto. Impact of ultralight axion self-interactions on the large scale structure of the universe. Physical Review D, 97(2), Jan 2018.

[55] M. Dine, W. Fischler, and M. Srednicki. A simple solution to the strong CP problem with a harmless axion. Phys. Lett. B, 104:199-202, August 1981. 
[56] Michael Dine and Willy Fischler. The Not So Harmless Axion. Phys. Lett. B, 120:137-141, 1983.

[57] Marco Drewes. The Phenomenology of Right Handed Neutrinos. International Journal of Modern Physics E, 22(8):1330019-593, August 2013.

[58] Xiaolong Du, Christoph Behrens, Jens C. Niemeyer, and Bodo Schwabe. Core-halo mass relation of ultralight axion dark matter from merger history. Phys. Rev. D, 95(4):043519, 2017.

[59] Joshua Eby, Chris Kouvaris, Niklas GrNielsen, and L.C.R. Wijewardhana. Boson Stars from Self-Interacting Dark Matter. JHEP, 02:028, 2016.

[60] Benedikt Eggemeier and Jens C. Niemeyer. Formation and mass growth of axion stars in axion miniclusters. Phys. Rev. D, 100(6):063528, 2019.

[61] Daniel J. Eisenstein, Idit Zehavi, David W. Hogg, et al. Detection of the Baryon Acoustic Peak in the Large-Scale Correlation Function of SDSS Luminous Red Galaxies. , 633(2):560-574, November 2005.

[62] H. I. Ewen and E. M. Purcell. Observation of a Line in the Galactic Radio Spectrum: Radiation from Galactic Hydrogen at 1,420 Mc./sec. , 168(4270):356, September 1951.

[63] R.P. Feynman. Chapter ii application of quantum mechanics to liquid helium. volume 1 of Progress in Low Temperature Physics, pages 17 -53. Elsevier, 1955.

[64] A. Finzi. On the validity of Newton's law at a long distance. ,127:21, January 1963.

[65] Bengt Fornberg. The pseudospectral method: Comparisons with finite differences for the elastic wave equation. Geophysics, 52(4):483, April 1987.

[66] J. A. Frieman, M. S. Turner, and D. Huterer. Dark energy and the accelerating universe. , 46:385-432, September 2008.

[67] Matteo Frigo and Steven G. Johnson. The fastest fourier transform in the west, 2018. 
[68] Steven R. Furlanetto, S. Peng Oh, and Elena Pierpaoli. Effects of dark matter decay and annihilation on the high-redshift $21 \mathrm{~cm}$ background. Physical Review D, 74(10), Nov 2006.

[69] R Genzel, D Hollenbach, and C H Townes. The nucleus of our galaxy. Reports on Progress in Physics, 57(5):417, 1994.

[70] Gian F. Giudice, Matthew McCullough, and Alfredo Urbano. Hunting for Dark Particles with Gravitational Waves. JCAP, 10:001, 2016.

[71] Noah Glennon and Chanda Prescod-Weinstein. Using pysiultralight to model scalar dark matter with self-interactions, 2020.

[72] David S. Graff and Katherine Freese. Analysis of a Hubble Space Telescope Search for Red Dwarfs: Limits on Baryonic Matter in the Galactic Halo. , 456:L49, January 1996.

[73] Michael B. Green, John H. Schwarz, and Edward Witten. Superstring theory. Volume 1 - Introduction. 1987.

[74] James E. Gunn and J. Richard Gott, III. On the Infall of Matter into Clusters of Galaxies and Some Effects on Their Evolution. Astrophys. J., 176:1-19, 1972.

[75] Alan H. Guth, Mark P. Hertzberg, and C. Prescod-Weinstein. Do dark matter axions form a condensate with long-range correlation? Physical Review D, 92(10), Nov 2015.

[76] Ulrich Heber. Extreme horizontal branch stars, 2008.

[77] T. Helfer, D. J. E. Marsh, K. Clough, M. Fairbairn, E. A. Lim, and R. Becerril. Black hole formation from axion stars. , 3:055, March 2017.

[78] G. Hinshaw, J. L. Weiland, R. S. Hill, et al. Five-Year Wilkinson Microwave Anisotropy Probe Observations: Data Processing, Sky Maps, and Basic Results. , 180(2):225-245, February 2009.

[79] Erik Holmberg. A Study of Double and Multiple Galaxies Together with Inquiries into some General Metagalactic Problems. Annals of the Observatory of Lund, 6:1-173, January 1937. 
[80] Edwin Hubble. A Relation between Distance and Radial Velocity among Extra-Galactic Nebulae. Proceedings of the National Academy of Science, 15(3):168-173, March 1929.

[81] Lam Hui, Jeremiah P. Ostriker, Scott Tremaine, and Edward Witten. Ultralight scalars as cosmological dark matter. Phys. Rev., D95(4):043541, 2017.

[82] Alejandro Ibarra and David Tran. Gamma-ray spectrum from gravitino dark matter decay. Phys. Rev. Lett., 100:061301, Feb 2008.

[83] Mustapha Ishak, James Richardson, David Garred, Delilah Whittington, Anthony Nwankwo, and Roberto Sussman. Dark energy or apparent acceleration due to a relativistic cosmological model more complex than the Friedmann-Lemaitre-Robertson-Walker model? ，78(12):123531, December 2008.

[84] J. C. Jackson. The dynamics of clusters of galaxies in universes with non-zero cosmological constant, and the virial theorem mass discrepancy. , 148:249, January 1970.

[85] Mubasher Jamil, D. Momeni, and Ratbay Myrzakulov. Resolution of dark matter problem in $\mathrm{f}(\mathrm{t})$ gravity. The European Physical Journal C, 72(8), Aug 2012.

[86] Prajwal Raj Kafle, Sanjib Sharma, Geraint F. Lewis, and Joss BlandHawthorn. On the Shoulders of Giants: Properties of the Stellar Halo and the Milky Way Mass Distribution. , 794(1):59, October 2014.

[87] M. Kamionkowski. WIMP and Axion Dark Matter. In E. Gava, A. Masiero, K. S. Narain, S. Randjbar-Daemi, G. Senjanovic, A. Smirnov, and Q. Shafi, editors, High Energy Physics and Cosmology, 1997 Summer School, volume 14, page 394, January 1998.

[88] David J. Kaup. Klein-gordon geon. Phys. Rev., 172:1331-1342, Aug 1968.

[89] B. W. Keller and J. W. Wadsley. $\Lambda$ CDM is Consistent with SPARC Radial Acceleration Relation. , 835(1):L17, January 2017.

[90] M. Khlopov, B.A. Malomed, and Ia.B. Zeldovich. Gravitational instability of scalar fields and formation of primordial black holes. , 215:575-589, 1985. 
[91] J. E. Kim. Weak-interaction singlet and strong CP invariance. , 43:103-107, July 1979.

[92] Kay Kirkpatrick, Anthony E. Mirasola, and Chanda Prescod-Weinstein. Relaxation times for Bose-Einstein condensation in axion miniclusters. Phys. Rev. D, 102(10):103012, 2020.

[93] Kay Kirkpatrick, Anthony E. Mirasola, and Chanda Prescod-Weinstein. Relaxation times for bose-einstein condensation in axion miniclusters, 2020.

[94] Felix Kling and Arvind Rajaraman. Towards an Analytic Construction of the Wavefunction of Boson Stars. Phys. Rev. D, 96(4):044039, 2017.

[95] Felix Kling and Arvind Rajaraman. Profiles of boson stars with selfinteractions. Phys. Rev. D, 97(6):063012, 2018.

[96] Edward W. Kolb and Igor I. Tkachev. Axion miniclusters and Bose stars. , 71(19):3051-3054, November 1993.

[97] E. Komatsu, J. Dunkley, M. R. Nolta, et al. Five-Year Wilkinson Microwave Anisotropy Probe Observations: Cosmological Interpretation. , 180(2):330376, February 2009.

[98] D. G. Levkov, A. G. Panin, and I. I. Tkachev. Relativistic Axions from Collapsing Bose Stars. Physical Review Letters, 118(1):011301, January 2017.

[99] D. G. Levkov, A. G. Panin, and I. I. Tkachev. Gravitational Bose-Einstein condensation in the kinetic regime. Phys. Rev. Lett., 121(15):151301, 2018.

[100] D.G. Levkov, A.G. Panin, and I.I. Tkachev. Relativistic axions from collapsing Bose stars. Phys. Rev. Lett., 118(1):011301, 2017.

[101] D.G. Levkov, A.G. Panin, and I.I. Tkachev. Relativistic axions from collapsing bose stars. Physical Review Letters, 118(1), Jan 2017.

[102] B. Lindblad. On the state of motion in the galactic system. , 87:553-564, May 1927.

[103] Mark R. Lovell, Carlos S. Frenk, Vincent R. Eke, Adrian Jenkins, Liang Gao, and Tom Theuns. The properties of warm dark matter haloes. , 439(1):300317, March 2014. 
[104] Andrea V. Macciò, Sinziana Paduroiu, Donnino Anderhalden, Aurel Schneider, and Ben Moore. Cores in warm dark matter haloes: a Catch 22 problem. , 424(2):1105-1112, August 2012.

[105] Andrea V. Macciò, Sinziana Paduroiu, Donnino Anderhalden, Aurel Schneider, and Ben Moore. Erratum: Cores in warm dark matter haloes: a Catch 22 problem. , 428(4):3715-3716, February 2013.

[106] Greg Madejski. Recent and Future Observations in the X-ray and Gammaray Bands: Chandra, Suzaku, GLAST, and NuSTAR. In Tomasz Bulik, Bronislaw Rudak, and Grzegorz Madejski, editors, Astrophysical Sources of High Energy Particles and Radiation, volume 801 of American Institute of Physics Conference Series, pages 21-30, November 2005.

[107] Mark S. Madsen and Andrew R. Liddle. The cosmological formation of boson stars. Physics Letters B, 251(4):507-510, November 1990.

[108] Juan Magaña and Tonatiuh Matos. A brief review of the scalar field dark matter model. Journal of Physics: Conference Series, 378:012012, Aug 2012.

[109] Magellan, U.Arizona, D.Clowe, et al. galaxy cluster 1e 0657-56, 2006.

[110] B. Majorovits. Madmax: A new road to axion dark matter detection, 2017.

[111] M. Markevitch, A. H. Gonzalez, D. Clowe, A. Vikhlinin, W. Forman, C. Jones, S. Murray, and W. Tucker. Direct Constraints on the Dark Matter SelfInteraction Cross Section from the Merging Galaxy Cluster 1E 0657-56. , 606(2):819-824, May 2004.

[112] D. J. E. Marsh. Axion cosmology. , 643:1-79, July 2016.

[113] D. J. E. Marsh and A.-R. Pop. Axion dark matter, solitons and the cusp-core problem. , 451:2479-2492, August 2015.

[114] David J.E. Marsh, Kin Chung Fong, Erik W. Lentz, Libor Šmejkal, and Mazhar N. Ali. Proposal to detect dark matter using axionic topological antiferromagnets. Physical Review Letters, 123(12), Sep 2019.

[115] Andrei Matlashov, Matthias Schmelz, Vyacheslav Zakosarenko, Ronny Stolz, and Yannis K. Semertzidis. Squid amplifiers for axion search experiments. Cryogenics, 91:125-127, 2018. 
[116] Tonatiuh Matos and L. Arturo Ureña-López. Further analysis of a cosmological model with quintessence and scalar dark matter. ,63(6):063506, March 2001.

[117] Tonatiuh Matos and L. Arturo Ureña-López. LETTER TO THE EDITOR: Quintessence and scalar dark matter in the Universe. Classical and Quantum Gravity, 17(13):L75-L81, July 2000.

[118] N. U. Mayall and L. H. Aller. The Rotation of the Spiral Nebula Messier 33. , 95:5, January 1942.

[119] M. Membrado, A. F. Pacheco, and J. Sañudo. Hartree solutions for the selfYukawian boson sphere. , 39(8):4207-4211, April 1989.

[120] David Merritt. Dark matter at the centers of galaxies. arXiv e-prints, page arXiv:1001.3706, January 2010.

[121] Eckehard W. Mielke and Franz E. Schunck. Boson stars: Alternatives to primordial black holes? Nucl. Phys., B564:185-203, 2000.

[122] Lino Miramonti. European underground laboratories: An overview. In Bruce Cleveland, Richard Ford, and Mark Chen, editors, Topical Workshop on Low Radioactivity Techniques: LRT 2004., volume 785 of American Institute of Physics Conference Series, pages 3-11, September 2005.

[123] Philip Mocz and Sauro Succi. Numerical solution of the nonlinear Schrödinger equation using smoothed-particle hydrodynamics. Phys. Rev. E, 91(5):053304, 2015.

[124] Philip Mocz, Mark Vogelsberger, Victor H. Robles, Jesús Zavala, Michael Boylan-Kolchin, Anastasia Fialkov, and Lars Hernquist. Galaxy formation with becdm - i. turbulence and relaxation of idealized haloes. Monthly Notices of the Royal Astronomical Society, 471(4):4559-4570, Jul 2017.

[125] Nathan Musoke, Shaun Hotchkiss, and Richard Easther. Lighting the Dark: Evolution of the Postinflationary Universe. Phys. Rev. Lett., 124(6):061301, 2020.

[126] Joan R. Najita, Glenn P. Tiede, and John S. Carr. From Stars to Superplanets: The Low-Mass Initial Mass Function in the Young Cluster IC 348. , 541(2):977-1003, October 2000. 
[127] Priyamvada Natarajan, Urmila Chadayammuri, Mathilde Jauzac, et al. Mapping substructure in the HST Frontier Fields cluster lenses and in cosmological simulations. , 468(2):1962-1980, June 2017.

[128] Julio F. Navarro, Carlos S. Frenk, and Simon D. M. White. The Structure of Cold Dark Matter Halos. , 462:563, May 1996.

[129] Robert J. Nemiroff and Bijunath Patla. Adventures in Friedmann cosmology: A detailed expansion of the cosmological Friedmann equations. American Journal of Physics, 76(3):265-276, March 2008.

[130] Jens C. Niemeyer and Richard Easther. Inflaton clusters and inflaton stars. Journal of Cosmology and Astroparticle Physics, 2020(07):030-030, Jul 2020.

[131] C. Nore, M. Abid, and M. E. Brachet. Kolmogorov turbulence in lowtemperature superflows. Phys. Rev. Lett., 78:3896-3899, May 1997.

[132] Ciaran O'HARE. Axion photon with projections, 2020.

[133] J. H. Oort. Observational evidence confirming Lindblad's hypothesis of a rotation of the galactic system. ,3:275, April 1927.

[134] J. M. Overduin and P. S. Wesson. Dark matter and background light. , 402(56):267-406, November 2004.

[135] R. D. Peccei and Helen R. Quinn. CP conservation in the presence of pseudoparticles. Phys. Rev. Lett., 38:1440-1443, Jun 1977.

[136] P. J. Peebles and Bharat Ratra. The cosmological constant and dark energy. Reviews of Modern Physics, 75(2):559-606, April 2003.

[137] P. J. E. Peebles and J. T. Yu. Primeval Adiabatic Perturbation in an Expanding Universe. , 162:815, December 1970.

[138] A. A. Penzias and R. W. Wilson. A Measurement of Excess Antenna Temperature at 4080 Mc/s. , 142:419-421, July 1965.

[139] Will J. Percival, Shaun Cole, Daniel J. Eisenstein, Robert C. Nichol, John A. Peacock, Adrian C. Pope, and Alexander S. Szalay. Measuring the Baryon Acoustic Oscillation scale using the Sloan Digital Sky Survey and 2dF Galaxy Redshift Survey. , 381(3):1053-1066, November 2007. 
[140] S. Perlmutter, G. Aldering, G. Goldhaber, R. A. Knop, P. Nugent, P. G. Castro, S. Deustua, S. Fabbro, A. Goobar, D. E. Groom, and et al. Measurements of and from 42 high-redshift supernovae. The Astrophysical Journal, 517(2):565-586, Jun 1999.

[141] E. Bruce Pitman, M.Sc. Katia, A. Rona, et al. Internet resources for the finite difference method for pde's, Jul 7, 2004 - Jun 22, 2017.

[142] Planck Collaboration, P. A. R. Ade, N. Aghanim, M. I. R. Alves, C. ArmitageCaplan, M. Arnaud, M. Ashdown, F. Atrio-Barandela, J. Aumont, H. Aussel, and et al. Planck 2013 results. I. Overview of products and scientific results. ,571:A1, November 2014.

[143] Planck Collaboration, P. A. R. Ade, and N. and Aghanim. Planck 2015 results. XIII. Cosmological parameters. , 594:A13, September 2016.

[144] John Preskill, Mark B. Wise, and Frank Wilczek. Cosmology of the Invisible Axion. Phys. Lett. B, 120:127-132, 1983.

[145] Adam G. Riess, Alexei V. Filippenko, Peter Challis, et al. Observational Evidence from Supernovae for an Accelerating Universe and a Cosmological Constant. , 116(3):1009-1038, September 1998.

[146] Adam G. Riess, Alexei V. Filippenko, Peter Challis, et al. Observational evidence from supernovae for an accelerating universe and a cosmological constant. The Astronomical Journal, 116(3):1009-1038, Sep 1998.

[147] Victor H Robles, James S Bullock, and Michael Boylan-Kolchin. Scalar field dark matter: helping or hurting small-scale problems in cosmology? Monthly Notices of the Royal Astronomical Society, 483(1):289-298, Nov 2018.

[148] D. H. Rogstad and G. S. Shostak. Gross Properties of Five Scd Galaxies as Determined from 21-CENTIMETER Observations. , 176:315, September 1972.

[149] Pablo A. Rosado, Paul D. Lasky, Eric Thrane, Xingjiang Zhu, Ilya Mandel, and Alberto Sesana. Detectability of Gravitational Waves from HighRedshift Binaries. , 116(10):101102, March 2016.

[150] Pilar Ruiz-Lapuente. Dark Energy: Observational and Theoretical Approaches. 2010. 
[151] Gray Rybka, Andrew Wagner, Kunal Patel, Robert Percival, Katleiah Ramos, and Aryeh Brill. Search for dark matter axions with the Orpheus experiment. , 91(1):011701, January 2015.

[152] V. Sahni and L. Wang. New cosmological model of quintessence and dark matter. , 62(10):103517, November 2000.

[153] Richard Schaeffer and Joseph Silk. Cold, Warm, or Hot Dark Matter: Biased Galaxy Formation and Pancakes. , 332:1, September 1988.

[154] H.-Y. Schive, T. Chiueh, and T. Broadhurst. Cosmic structure as the quantum interference of a coherent dark wave. Nature Physics, 10:496-499, July 2014.

[155] H.-Y. Schive, M.-H. Liao, T.-P. Woo, S.-K. Wong, T. Chiueh, T. Broadhurst, and W.-Y. P. Hwang. Understanding the Core-Halo Relation of Quantum Wave Dark Matter from 3D Simulations. , 113(26):261302, December 2014.

[156] Hsi-Yu Schive, Tzihong Chiueh, and Tom Broadhurst. Cosmic structure as the quantum interference of a coherent dark wave. Nature Physics, 10(7):496499, July 2014.

[157] Bodo Schwabe, Jens C. Niemeyer, and Jan F. Engels. Simulations of solitonic core mergers in ultralight axion dark matter cosmologies. Phys. Rev., D94(4):043513, 2016.

[158] Edward Seidel and Wai-Mo Suen. Dynamical evolution of boson stars: Perturbing the ground state. Phys. Rev. D, 42:384-403, Jul 1990.

[159] C. D. Shane and C. A. Wirtanen. The distribution of extragalactic nebulae. , 59:285-304, September 1954.

[160] M. A. Shifman, A. I. Vainshtein, and V. I. Zakharov. Can confinement ensure natural CP invariance of strong interactions? Nuclear Physics B, 166:493-506, April 1980.

[161] G. Seth Shostak. Aperture synthesis observations of neutral hydrogen in three galaxies. PhD thesis, California Institute of Technology, January 1972.

[162] P. Sikivie. Dark Matter Axions. International Journal of Modern Physics A, 25(2-03):554-563, January 2010. 
[163] P. Sikivie, N. Sullivan, and D. B. Tanner. Proposal for Axion Dark Matter Detection Using an LC Circuit. , 112(13):131301, April 2014.

[164] Pierre Sikivie. Axion Cosmology. Lect. Notes Phys., 741:19-50, 2008.

[165] C. Skordis, D. F. Mota, P. G. Ferreira, and C. Bœhm. Large Scale Structure in Bekenstein's Theory of Relativistic Modified Newtonian Dynamics. , 96(1):011301, January 2006.

[166] L. Skrbek. Quantum turbulence. In Journal of Physics Conference Series, volume 318 of Journal of Physics Conference Series, page 012004, December 2011.

[167] Sinclair Smith. The Mass of the Virgo Cluster. , 83:23, January 1936.

[168] Yvette Smith. Making sense of the big bang: Wilkinson microwave anisotropy probe, 2017.

[169] Igor I. Smolyaninov. Oscillating cosmological force modifies Newtonian dynamics. Galaxies, 8(2):45, 2020.

[170] Volker Springel, Simon D. M. White, Adrian Jenkins, Carlos S. Frenk, Naoki Yoshida, Liang Gao, Julio Navarro, Robert Thacker, Darren Croton, John Helly, and et al. Simulations of the formation, evolution and clustering of galaxies and quasars. Nature, 435(7042):629-636, Jun 2005.

[171] R. A. Sunyaev and Ya. B. Zeldovich. Small-Scale Fluctuations of Relic Radiation. , 7(1):3-19, April 1970.

[172] Abril Suárez, Victor H. Robles, and Tonatiuh Matos. A Review on the Scalar Field/Bose-Einstein Condensate Dark Matter Model. Astrophys. Space Sci. Proc., 38:107-142, 2014.

[173] A. N. Taylor, S. Dye, T. J. Broadhurst, N. Benítez, and E. van Kampen. Gravitational Lens Magnification and the Mass of Abell 1689. , 501(2):539-553, July 1998.

[174] James E. Taylor. Dark matter halos from the inside out. Advances in Astronomy, 2011:1-17, 2011.

[175] NASA/WMAP Science Team. Nine year microwave sky, 2014. 
[176] P. Tisserand, L. Le Guillou, C. Afonso, et al. Limits on the Macho content of the Galactic Halo from the EROS-2 Survey of the Magellanic Clouds. , 469(2):387-404, July 2007.

[177] Diego F. Torres, S. Capozziello, and G. Lambiase. Supermassive boson star at the galactic center? Phys. Rev. D, 62:104012, Oct 2000.

[178] Scott Tremaine and James E. Gunn. Dynamical role of light neutral leptons in cosmology. , 42(6):407-410, February 1979.

[179] Christos G. Tsagas. Peculiar motions, accelerated expansion, and the cosmological axis. , 84(6):063503, September 2011.

[180] Cora Uhlemann, Michael Kopp, and Thomas Haugg. Schrödinger method as N-body double and UV completion of dust. , 90(2):023517, 2014.

[181] Sidney van den Bergh. The stability of clusters of galaxies. , 66:566, December 1961.

[182] Jan Veltmaat, Jens C. Niemeyer, and Bodo Schwabe. Formation and structure of ultralight bosonic dark matter halos. Phys. Rev. D, 98(4):043509, 2018.

[183] F.H. Vincent, Z. Meliani, P. Grandclement, E. Gourgoulhon, and O. Straub. Imaging a boson star at the Galactic center. Class. Quant. Grav., 33(10):105015, 2016.

[184] C. F. von Weizsäcker. The Evolution of Galaxies and Stars. , 114:165, September 1951.

[185] Matthew G. Walker and Jorge Peñarrubia. A METHOD FOR MEASURING (SLOPES OF) THE MASS PROFILES OF DWARF SPHEROIDAL GALAXIES. The Astrophysical Journal, 742(1):20, nov 2011.

[186] David H. Weinberg, James S. Bullock, Fabio Governato, Rachel Kuzio de Naray, and Annika H. G. Peter. Cold dark matter: Controversies on small scales. Proceedings of the National Academy of Sciences, 112(40):12249-12255, Feb 2015.

[187] Steven Weinberg. A New Light Boson? , 40:223-226, 1978.

[188] Lawrence M. Widrow and Nick Kaiser. Using the Schroedinger Equation to Simulate Collisionless Matter. , 416:L71, October 1993. 
[189] Frank Wilczek. Problem of Strong $\mathrm{p}$ and $\mathrm{t}$ Invariance in the Presence of Instantons. , 40:279-282, 1978.

[190] David L. Wiltshire. Cosmological equivalence principle and the weak-field limit. , 78(8):084032, October 2008.

[191] Edward Witten. Some properties of O(32) superstrings. Physics Letters B, 149(4-5):351-356, December 1984.

[192] N. J. Woolf. On the Stabilization of Clusters of Galaxies by Ionized Gas. , 148:287, April 1967.

[193] Xiang-Ping Wu, Tzihong Chiueh, Li-Zhi Fang, and Yan-Jie Xue. A comparison of different cluster mass estimates: consistency or discrepancy? , 301(3):861-871, December 1998.

[194] Yu-Cheng Wu, Xi-Qing Hao, Qian Yue, et al. Measurement of cosmic ray flux in the China JinPing underground laboratory. Chinese Physics C, 37(8):086001, August 2013.

[195] L. Wyrzykowski, J. Skowron, S. Kozłowski, A. Udalski, M. K. Szymański, M. Kubiak, G. Pietrzyński, I. Soszyński, O. Szewczyk, K. Ulaczyk, R. Poleski, and P. Tisserand. The OGLE view of microlensing towards the Magellanic Clouds - IV. OGLE-III SMC data and final conclusions on MACHOs. , 416(4):2949-2961, October 2011.

[196] Xiaoying Xu, Antonio J. Cuesta, Nikhil Padmanabhan, Daniel J. Eisenstein, and Cameron K. McBride. Measuring $\mathrm{D}_{A}$ and $\mathrm{H}$ at $\mathrm{z}=0.35$ from the SDSS DR7 LRGs using baryon acoustic oscillations. , 431(3):2834-2860, May 2013.

[197] Bal Krishna Yadav and Murli Manohar Verma. Dark matter as scalaron in $\mathrm{f}(\mathrm{r})$ gravity models. Journal of Cosmology and Astroparticle Physics, 2019(10):052-052, Oct 2019.

[198] A.R. Zhitnitsky. On Possible Suppression of the Axion Hadron Interactions. (In Russian). Sov.J . Nucl. Phys., 31:260, 1980.

[199] T. G Zlosnik, P. G Ferreira, and G. D. Starkman. Modifying gravity with the aether: An alternative to dark matter. Phys. Rev. D, 75:044017, Feb 2007. 
[200] F. Zwicky. Die Rotverschiebung von extragalaktischen Nebeln. Helvetica Physica Acta, 6:110-127, January 1933.

[201] F. Zwicky. Multiple Galaxies. Ergebnisse der exakten Naturwissenschaften, 29:344-385, January 1956. 